quatrième série-tome 42 fascicule 4 juillet-août 2009

$$
\begin{aligned}
& \text { ANNALES } \\
& \text { SCIENTIFIQUES } \\
& \text { de } \\
& \text { L'ECOLE } \\
& \text { NORMALE } \\
& \text { SUPÉRIEURE }
\end{aligned}
$$

Takashi TSUBOI

On the group of real analytic diffeomorphisms 
Ann. Scient. Éc. Norm. Sup.

$4^{\mathrm{e}}$ série, t. 42,2009, p. 601 à 651

\title{
ON THE GROUP OF REAL ANALYTIC DIFFEOMORPHISMS
}

\author{
BY TAKASHI TSUBOI
}

\begin{abstract}
The group of real analytic diffeomorphisms of a real analytic manifold is a rich group. It is dense in the group of smooth diffeomorphisms. Herman showed that for the $n$-dimensional torus, its identity component is a simple group. For $U(1)$ fibered manifolds, for manifolds admitting special semi-free $U(1)$ actions and for 2- or 3-dimensional manifolds with nontrivial $U(1)$ actions, we show that the identity component of the group of real analytic diffeomorphisms is a perfect group.

RÉsUMÉ. - Le groupe des difféomorphismes analytiques réels d'une variété analytique réelle est un groupe riche. Il est dense dans le groupe des difféomorphismes lisses. Herman a montré que, pour le tore de dimension $n$, sa composante connexe de l'identité est un groupe simple. Pour les variétés $U(1)$ fibrées, pour les variétés admettant une action semi-libre spéciale de $U(1)$, et pour les variétés de dimension 2 ou 3 admettant une action non-triviale de $U(1)$, on montre que la composante de l'identité du groupe des difféomorphismes analytiques réels est un groupe parfait.
\end{abstract}

\section{Introduction and statement of the result}

Let $\operatorname{Diff}^{\omega}(M)$ denote the group of real analytic diffeomorphisms of a real analytic manifold $M$. The group $\operatorname{Diff}^{\omega}(M)$ is an open subset of the space of real analytic maps $\operatorname{Map}^{\omega}(M, M)$ with the $C^{1}$ topology. The group $\operatorname{Diff}^{\omega}(M)$ with the $C^{1}$ topology has a manifold structure modelled on the space $\mathcal{X}^{\omega}(M)$ of real analytic vector fields on $M$. Hence $\operatorname{Diff}^{\omega}(M)$ is locally contractible (see Proposition 11.9). It is well-known that $\operatorname{Diff}^{\omega}(M)$ is dense in the group Diff ${ }^{\infty}(M)$ of smooth diffeomorphisms in the $C^{1}$ topology (See Corollary 11.8). Hence $\operatorname{Diff}^{\omega}(M)$ is a huge complicated group.

Let $\operatorname{Diff}^{\omega}(M)_{0}$ denote the identity component of $\operatorname{Diff}^{\omega}(M)$. For the $n$-dimensional torus $T^{n}$, Herman [10] in 1974 showed that $\operatorname{Diff}^{\omega}\left(T^{n}\right)_{0}$ is a simple group. For 30 years since then,

The author is partially supported by Grant-in-Aid for Scientific Research 16204004, 17104001, 20244003, Grant-in-Aid for Exploratory Research 18654008, Japan Society for Promotion of Science, and by the $21^{\text {st }}$ Century COE Program and the Global COE Program at Graduate School of Mathematical Sciences, the University of Tokyo. 
there are no new results on the simplicity of the groups of real analytic diffeomorphisms. However, Herman conjectured and we may still conjecture that for any compact connected manifold $M$, the identity component $\operatorname{Diff}^{\omega}(M)_{0}$ of the group of real analytic diffeomorphisms is simple.

Now, in this paper, we change the question. If an infinite group is simple, then it is perfect. Hence we may ask a weaker question and may try to show the perfectness of the group of real analytic diffeomorphisms. Note that, in the case of the group of smooth diffeomorphisms, the perfectness implies the simplicity ([5], see also [2]), however, we cannot apply this argument to the group of real analytic diffeomorphisms.

For this question, our present results are as follows.

THEOREM 1.1. - Let $M$ be a real analytically $U(1)$ fibered real analytic closed manifold. Then the identity component $\operatorname{Diff}^{\omega}(M)_{0}$ of the group of real analytic diffeomorphisms of $M$ is a perfect group.

We consider other manifolds with well-understood $U(1)$ actions. Let $N$ be a compact $(n-1)$-dimensional manifold with boundary $\partial N$. Let $M$ be the $n$-dimensional manifold obtained from $N \times U(1)$ by identifying $\{x\} \times U(1)$ to a point for $x \in \partial N$. This $M$ has a real analytic structure with the obvious real analytic $U(1)$ action. We call this $U(1)$ action a special semi-free $U(1)$ action. Spheres and direct products with spheres admit special semi-free $U(1)$ actions.

THEOREM 1.2. - Let $M$ be a real analytic manifold which admits a special semi-free $U(1)$ action. Then the identity component $\operatorname{Diff}^{\omega}(M)_{0}$ of the group of real analytic diffeomorphisms of $M$ is a perfect group.

If the dimension of $M$ is 2 or 3 , we can show the perfectness of $\operatorname{Diff}^{\omega}(M)_{0}$ if $M$ admits a nontrivial $U(1)$ action.

THeorem 1.3. - Let $M$ be a real analytic manifold of dimension 2 or 3 which admits a nontrivial $U(1)$ action. Then the identity component $\operatorname{Diff}^{\omega}(M)_{0}$ of the group of real analytic diffeomorphisms of $M$ is a perfect group.

These theorems are shown in the following way.

First, we show the perfectness of the group of orbit preserving diffeomorphisms for the $U(1)$ bundles (Theorem 2.2) and a similar result for the orbit preserving diffeomorphisms for the manifolds admitting special semi-free $U(1)$ actions (Theorem 5.1). These theorems for orbit preserving diffeomorphisms are proved by using the famous Arnold theorem [1] for the Diophantine rotations and a similar Theorem 5.3 for the rotations of concentric circles, which we prove in Section 10. We also need certain explicit orbitwise actions of elements of $S L(2 ; \boldsymbol{R})$, and the existence of such nice actions gives the restriction to the $U(1)$ actions for which we can show our results by now.

To show our main theorems, we perturb the given $U(1)$ action by real analytic diffeomorphisms and obtain finitely many $U(1)$ actions such that the tangent space $T_{x} M^{n}$ of any point $x$ of the manifold $M^{n}$ is spanned by the generating vector fields of the resultant $U(1)$ actions.

$4^{\text {e }}$ SÉRIE - TOME $42-2009-\mathrm{N}^{\mathrm{o}} 4$ 
For $n=\operatorname{dim}\left(M^{n}\right)$ and $U(1)$ actions generated by the vector fields $\xi_{1}, \ldots, \xi_{n}$, we have

the determinant $\Delta=\operatorname{det}\left(\xi_{i j}\right)$ with respect to an orthonormal frame $\frac{\partial}{\partial x_{j}}$ for a real analytic Riemannian metric on $M^{n}$, where $\xi_{i}=\sum_{j=1}^{n} \xi_{i j} \frac{\partial}{\partial x_{j}}(i=1, \ldots, n)$.

On the open set where $\Delta \neq 0$, a diffeomorphism sufficiently close to the identity can be written as a composition of orbit preserving diffeomorphisms. In fact, we show that for real analytic diffeomorphisms $f$ such that $f-$ id are divisible by a certain power of $\Delta, f$ can be written as a composition of orbit preserving real analytic diffeomorphisms. This is done by an inverse mapping theorem for real analytic maps with singular Jacobians (Theorems 6.7 or 6.12).

Now we need to decompose a real analytic diffeomorphism sufficiently close to the identity as a composition of real analytic diffeomorphisms which satisfy the assumption of Theorems 6.7 or 6.12. This is done by using the regimentation Lemma 7.1, which replaces the fragmentation lemma ([2], [13]) for the smooth diffeomorphisms.

Then we use the perfectness of the group of orbit preserving diffeomorphisms of $U(1)$ bundles (Theorem 2.2) or a similar theorem (Theorem 5.1) for manifolds admitting special semi-free actions, to show our main theorems (Section 8).

Our method can treat the real analytic manifolds with a little more general $U(1)$ actions. We say that two elements of a group are homologous if they represent the same element in the abelianization of the group. In Section 9, we show that, if the manifold admits a nontrivial $U(1)$ action, any real analytic diffeomorphism isotopic to the identity is homologous to a diffeomorphism which is an orbitwise rotation (Proposition 9.1). Then we show Theorem 1.3 by showing Propositions 9.2, 9.3 and Theorem 9.4.

We think that $\operatorname{Diff}^{\omega}(M)_{0}$ is perfect if $M$ admits a nontrivial $U(1)$ action. But for the moment we need a structure theorem for the orbifold $M / U(1)$ in the construction of a nice multi-section outside of a codimension 2 suborbifold to show that orbitwise rotations are homologous to zero.

\section{Orbit preserving diffeomorphisms of $U(1)$ bundles}

As we mentioned, for the $n$-dimensional torus $T^{n}$, Herman [10] in 1974 noticed that the result of Arnold [1] implies Diff ${ }^{\omega}\left(T^{n}\right)_{0}$ is a simple group. Hence it is perfect.

We note that Herman's proof ([10]) uses the fact that the commutator subgroup $\left[\operatorname{Diff}^{\omega}\left(T^{n}\right)_{0}, \operatorname{Diff}^{\omega}\left(T^{n}\right)_{0}\right]$ of $\operatorname{Diff}^{\omega}\left(T^{n}\right)_{0}$ is its dense subgroup. In fact, for the group Diff ${ }^{\infty}(M)$ of $C^{\infty}$ diffeomorphisms of a smooth manifold $M$, its identity component $\operatorname{Diff}^{\infty}(M)_{0}$ is perfect by the result of Thurston ([20], [2]). Since Diff ${ }^{\omega}(M)$ is dense in $\operatorname{Diff}^{\infty}(M)$, the commutator subgroup $\left[\operatorname{Diff}^{\omega}(M)_{0}, \operatorname{Diff}^{\omega}(M)_{0}\right]$ is dense in $\operatorname{Diff}^{\omega}(M)_{0}$.

For the real analytic diffeomorphisms of $T^{n}$, Arnold [1] already noticed the followings.

Theorem 2.1 (Arnold[1]). - Let $\alpha \in \boldsymbol{R}^{n}$ satisfy the Diophantine condition. For a real analytic family $\Phi(w)(w \in W)$ of analytic diffeomorphisms of $T^{n}$ close to the identity, there is an analytic family $(\psi(w), \lambda(w)) \in \operatorname{Diff}^{\omega}\left(T^{n}\right)_{0} \times T^{n}$ such that

$$
\Phi(w)=R_{\lambda(w)-\alpha} \circ \psi(w) \circ R_{\alpha} \circ \psi(w)^{-1},
$$


where $R_{*}$ denotes the rotation by $*$ on $T^{n}=\boldsymbol{R}^{n} / \boldsymbol{Z}^{n}$.

Here a real vector $\alpha \in \boldsymbol{R}^{n}$ is said to satisfy the Diophantine condition if there exist positive real numbers $C$ and $\beta$ such that $|\alpha \bullet \ell-m| \geq C\|\ell\|^{-\beta}$ for any $\ell \in \boldsymbol{Z}^{n} \backslash\{0\}$ and $m \in \boldsymbol{Z}$.

Since $R_{\lambda}$ can be written as a commutator in

$$
P S L(2 ; \boldsymbol{R})^{n}=P S L(2 ; \boldsymbol{R}) \times \cdots \times P S L(2 ; \boldsymbol{R})
$$

depending real analytically on $\lambda, \Phi(w)$ can be written as a product of 2 commutators depending real analytically on $w \in W$.

This means that for a compact manifold $N$ and the product $N \times T^{n}$ with the product foliation $\mathcal{F}=\left(\{*\} \times T^{n}\right)(* \in N)$, the group $\operatorname{Diff}^{\omega}(\mathcal{F})_{0}$ is a perfect group, where $\operatorname{Diff}^{\omega}(\mathcal{F})$ denotes the group of real analytic diffeomorphisms mapping each fiber of the projection $N \times T^{n} \longrightarrow N$ to itself and the subscript ${ }_{0}$ denotes the identity component.

We first generalize the perfectness result for the group of orbit preserving diffeomorphisms of a $U(1)$ bundle.

TheOREM 2.2. - Let $p: M \longrightarrow B$ be a real analytic principal $U(1)$ bundle over a closed manifold $B$. Let $\operatorname{Diff}^{\omega}(\mathcal{F})$ denote the group of real analytic diffeomorphisms mapping each fiber of the projection $p: M \longrightarrow B$ to itself. The identity component $\operatorname{Diff}^{\omega}(\mathcal{F})_{0}$ of $\operatorname{Diff}^{\omega}(\mathcal{F})$ is a perfect group.

\section{Proof of Theorem 2.2}

Proof of Theorem 2.2 for trivial $U(1)$ bundles. - Theorem 2.2 for the trivial $U(1)$ bundle is just a reformation of Arnold's Theorem 2.1. In this case, $M=B \times U(1)$ and $\mathcal{F}=(\{*\} \times U(1))_{* \in B}$. An element of $\operatorname{Diff}^{\omega}(\mathcal{F})_{0}$ is written as the real analytic family $\Phi(w)$ $(w \in B)$ of real analytic diffeomorphism of $U(1)$. It is enough to show that $\Phi(w)$ near the identity can be written as a product of commutators.

Take a Diophantine rotation $R_{\alpha}$ in the direction of the fibers of the $U(1)$ bundle. The element $\Phi(w)$ near the identity is written as

$$
\Phi(w)=R_{\lambda(w)-\alpha} \circ \psi(w) \circ R_{\alpha} \circ \psi(w)^{-1} .
$$

Here, $\lambda(w)$ is determined uniquely by the condition that the rotation number of $R_{-\lambda(w)} \circ\left(R_{\alpha} \circ \Phi(w)\right)$ coincides with that of $R_{\alpha}, \alpha \bmod 1$. In the proof in [1] of Arnold's Theorem 2.1, the conjugating diffeomorphism $\psi(w)$ is obtained uniquely so that the base point $1 \in U(1)$ is fixed $(\psi(w)(1)=1)$. Thus $\psi(w)(w \in B)$ is a real analytic family of real analytic diffeomorphisms. In the expression $\Phi(w)=R_{\lambda(w)-\alpha} \circ \psi(w) \circ R_{\alpha} \circ \psi(w)^{-1}, R_{\lambda(w)}$ can be written as a product of two commutators in $\operatorname{Map}^{\omega}(B, S L(2, \boldsymbol{R}))$ by the following Lemma 3.1. Thus Theorem 2.2 for trivial $U(1)$ bundles is shown.

Lemma 3.1. - A rotation $\left(\begin{array}{cc}X & -Y \\ Y & X\end{array}\right)\left(X^{2}+Y^{2}=1\right)$ close to the identity can be written as a product of 2 commutators using products of rotations and diagonal matrices. 
Proof. - We notice the following equality:

$$
\begin{aligned}
& \left(\begin{array}{cc}
X & -Y \\
Y & X
\end{array}\right)\left(\begin{array}{cc}
a & 0 \\
0 & \frac{1}{a}
\end{array}\right)\left(\begin{array}{cc}
X & \frac{2 a^{2} Y}{a^{4}-1} \\
\frac{2 a^{2} Y}{a^{4}-1} & X
\end{array}\right)\left(\begin{array}{cc}
\frac{1}{a} & 0 \\
0 & a
\end{array}\right) \\
& =\left(\begin{array}{cc}
\frac{1}{a} & 0 \\
0 & a
\end{array}\right)\left(\begin{array}{cc}
X & \frac{2 a^{2} Y}{a^{4}-1} \\
\frac{2 a^{2} Y}{a^{4}-1} & X
\end{array}\right)\left(\begin{array}{ll}
a & 0 \\
0 & \frac{1}{a}
\end{array}\right)\left(\begin{array}{cc}
X & Y \\
-Y & X
\end{array}\right) .
\end{aligned}
$$

Put

$$
\left(\begin{array}{cc}
W & Z \\
Z & W
\end{array}\right)=\left(1 / \sqrt{1-\frac{\left(a^{4}+1\right)^{2} Y^{2}}{\left(a^{4}-1\right)^{2}}}\right)\left(\begin{array}{cc}
X & \frac{2 a^{2} Y}{a^{4}-1} \\
\frac{2 a^{2} Y}{a^{4}-1} & X
\end{array}\right) \in S L(2 ; \boldsymbol{R}) .
$$

Then

$$
\begin{aligned}
\left(\begin{array}{cc}
X & -Y \\
Y & X
\end{array}\right)^{2}= & \left(\begin{array}{ll}
\frac{1}{a} & 0 \\
0 & a
\end{array}\right)\left(\begin{array}{cc}
W & Z \\
Z & W
\end{array}\right)\left(\begin{array}{ll}
a & 0 \\
0 & \frac{1}{a}
\end{array}\right)\left(\begin{array}{cc}
X & Y \\
-Y & X
\end{array}\right) \\
& \cdot\left(\begin{array}{ll}
a & 0 \\
0 & \frac{1}{a}
\end{array}\right)\left(\begin{array}{cc}
W & -Z \\
-Z & W
\end{array}\right)\left(\begin{array}{ll}
\frac{1}{a} & 0 \\
0 & a
\end{array}\right)\left(\begin{array}{cc}
X & -Y \\
Y & X
\end{array}\right) \\
= & {\left[\left(\begin{array}{cc}
\frac{1}{a} & 0 \\
0 & a
\end{array}\right),\left(\begin{array}{cc}
W & Z \\
Z & W
\end{array}\right)\right]\left[\left(\begin{array}{cc}
W & Z \\
Z & W
\end{array}\right),\left(\begin{array}{cc}
X & Y \\
-Y & X
\end{array}\right)\left(\begin{array}{ll}
a & 0 \\
0 & \frac{1}{a}
\end{array}\right)\right] . }
\end{aligned}
$$

Hence $\left(\begin{array}{cc}X & -Y \\ Y & X\end{array}\right)^{2}$ can be written as a product of 2 commutators. Here note that

$$
\left(\begin{array}{cc}
W & Z \\
Z & W
\end{array}\right)=\left(\begin{array}{cc}
\frac{1}{\sqrt{2}} & -\frac{1}{\sqrt{2}} \\
\frac{1}{\sqrt{2}} & \frac{1}{\sqrt{2}}
\end{array}\right)\left(\begin{array}{cc}
W+Z & 0 \\
0 & W-Z
\end{array}\right)\left(\begin{array}{cc}
\frac{1}{\sqrt{2}} & \frac{1}{\sqrt{2}} \\
-\frac{1}{\sqrt{2}} & \frac{1}{\sqrt{2}}
\end{array}\right)
$$

and this shows the lemma.

Proof of Theorem 2.2 for non-trivial U(1) bundles. - We choose a family of trivializing neighborhoods for the $U(1)$ bundle. On each trivializing neighborhood, we can write $\Phi(w)$ as a family of real analytic diffeomorphisms. Then we take a Diophantine rotation $R_{\alpha}$ and $\Phi(w)$ near the identity can be written as $\Phi(w)=R_{\lambda(w)-\alpha} \circ \psi(w) \circ R_{\alpha} \circ \psi(w)^{-1}$ on the trivializing neighborhood.

Now note that, for any $U(1)$ bundle, the rotation in the direction of the fiber is defined. Hence the Diophantine rotation $R_{\alpha}$ is globally defined. Moreover, since $\lambda(w)$ is determined uniquely by the condition that the rotation number of $R_{-\lambda(w)} \Phi(w)$ coincides with that of $R_{\alpha}$, $\alpha \bmod 1$, and this condition does not depend on the choice of local trivialization of the $U(1)$ bundle, $\lambda(w)$ is well defined as a real analytic function on $B$.

On the other hand, $\psi(w)$ on a trivializing neighborhood is unique only up to composing rotations on the right. However, the Lebesgue measure along the fiber is well defined, and among these $\psi(w)$, there is a unique element $\psi(w) \in \operatorname{Diff}^{\omega}(\mathcal{F})_{0}$ such that

$$
\int_{S^{1}}(\psi(w)-\mathrm{id})(\theta) \mathrm{d} \theta=0
$$

In fact, for a given $\psi(w)$, put

$$
\beta(w)=\int_{S^{1}}(\psi(w)-\mathrm{id})(\theta) \mathrm{d} \theta=-\int_{S^{1}}\left(\psi(w)^{-1}-\mathrm{id}\right)(\theta) \mathrm{d} \theta .
$$


Then

$$
\begin{aligned}
\int_{S^{1}}\left(\psi(w) \circ R_{-\beta(w)}-\mathrm{id}\right)(\theta) \mathrm{d} \theta & \\
= & \int_{S^{1}}\left(\psi(w) \circ R_{-\beta(w)}-\psi(w)+\psi(w)-\mathrm{id}\right)(\theta) \mathrm{d} \theta \\
= & -\int_{S^{1}}\left(R_{\beta} \circ \psi(w)^{-1}-\psi(w)^{-1}\right)(\theta) \mathrm{d} \theta+\int_{S^{1}}(\psi(w)-\mathrm{id})(\theta) \mathrm{d} \theta=0 .
\end{aligned}
$$

By replacing the given $\psi(w)$ by $\psi(w) \circ R_{-\beta(w)}$, the new $\psi(w)$ satisfies the above condition, and the conjugating diffeomorphism $\psi(w)$ taken for the trivializing neighborhoods for the $U(1)$ bundle matches up on the intersections.

Thus we can write $\Phi(w)=R_{\lambda(w)-\alpha} \circ \psi(w) \circ R_{\alpha} \circ \psi(w)^{-1}(w \in B)$. This means that any element of the abelianization $H_{1}\left(\operatorname{Diff}^{\omega}(\mathcal{F})_{0} ; \boldsymbol{Z}\right)$ is represented by an element of the group $\operatorname{Map}^{\omega}(B, U(1))_{0}$ of diffeomorphisms which are fiberwise rotation.

Rotations near the identity can be written as commutators in $P S L(2 ; \boldsymbol{R})$ or $S L(2 ; \boldsymbol{R})$, however we need a trivialization of the circle bundle to make $P S L(2 ; \boldsymbol{R})$ or $S L(2 ; \boldsymbol{R})$ act on the fiber.

We will show the following proposition, which completes the proof of Theorem 2.2 for non-trivial $U(1)$ bundles.

Proposition 3.2. - Each element of $\mathrm{Map}^{\omega}(B, U(1))_{0}$ can be written as a product of commutators of the group of real analytic orbit preserving diffeomorphisms $\operatorname{Diff}^{\omega}(\mathcal{F})_{0}$.

For the proof of Proposition 3.2, we carefully choose a finite family of trivializations of the circle bundle over the complements of submanifolds $D_{i}$ 's. We decompose an element of $\operatorname{Map}^{\omega}(B, U(1))_{0}$ into elements which are the identity over $D_{i}$. We see that there is an analytic action of $\operatorname{Map}^{\omega}\left(B \backslash D_{i}, S L(2 ; \boldsymbol{R})\right)$ on $p^{-1}\left(B \backslash D_{i}\right)$. The action of certain elements of $\operatorname{Map}^{\omega}\left(B \backslash D_{i}, S L(2 ; \boldsymbol{R})\right)$ extends to that of elements of $\operatorname{Map}^{\omega}\left(\left(B, D_{i}\right),(S L(2 ; \boldsymbol{R}),\{\right.$ id $\left.\})\right)$.

Proof of Proposition 3.2. - Let $p: M \longrightarrow B$ be a real analytic principal $U(1)$ bundle. Let $p_{E}: E \longrightarrow B$ be the associated complex line bundle. The space of real analytic sections of $E$ is a real vector space.

We use a finite set of real analytic sections $s_{i}: B \longrightarrow E(i=1, \ldots, k ; 2 k \geq n+1=$ $\operatorname{dim}(M)+1)$ transverse to the zero section such that $\bigcap_{i=1}^{k} s_{i}^{-1}(0)=\varnothing$. Then the bundle $M \longrightarrow B$ has $k$ trivializing open dense sets $B \backslash s_{i}^{-1}(0)$ which form a covering of $B$.

We consider a real analytic Hermitian metric for $E$. Then the square of the absolute value $\left|s_{i}\right|^{2}: B \longrightarrow \boldsymbol{R}(i=1, \ldots, k)$ is a non negative real analytic function on $B$.

For $r \in \operatorname{Map}^{\omega}(B, U(1))_{0}$ near the identity, let $\widetilde{r}: B \longrightarrow \widetilde{U(1)}$ be the lift near the identity, where $\widetilde{U(1)}$ is the universal covering group of $U(1)$.

Put

$$
\tilde{r}_{i}(w)=\left(\left|s_{i}\right|^{4}(w) / \sum_{j=1}^{k}\left|s_{j}\right|^{4}(w)\right) \frac{\widetilde{r}(w)}{2}
$$


where the product in $\widetilde{U(1)} \cong \boldsymbol{R}$ is written additively. Let $r_{i}$ be the element of $\operatorname{Map}^{\omega}(B, U(1))_{0}$ defined by $\widetilde{r}_{i}$. Then as an element of $\operatorname{Map}^{\omega}(B, U(1))_{0}$,

$$
r=r_{1}^{2} \cdots r_{k}^{2},
$$

where the product in $U(1)$ is written multiplicatively. Note that $r_{i}$ is flat along $s_{i}^{-1}(0)$ which is divisible by $\left|s_{i}\right|^{4}$.

For a real analytic section $s: B \longrightarrow E$ transverse to the zero section, $s^{-1}(0)$ is a codimension 2 submanifold of $B$. The restriction of the bundle $M \mid\left(B \backslash s^{-1}(0)\right)$ is trivialized by using the section $e: B \longrightarrow M$ given by $e(b)=\frac{s(b)}{|s(b)|}$. Then the coordinate transformation is written as follows. First,

$$
M \mid\left(B \backslash s_{i}^{-1}(0)\right) \longrightarrow\left(B \backslash s_{i}^{-1}(0)\right) \times U(1)
$$

is given by $x \longmapsto\left(p(x), \frac{x}{e_{i}(p(x))}\right)$, where $e_{i}=\frac{s_{i}(b)}{\left|s_{i}(b)\right|}$. Hence we have the coordinate transformation

$$
\begin{gathered}
\left(B \backslash s_{i}^{-1}(0)\right) \times U(1) \supset\left(B \backslash\left(s_{i}^{-1}(0) \cup s_{j}^{-1}(0)\right)\right) \times U(1) \\
\downarrow \\
\left(B \backslash s_{j}^{-1}(0)\right) \times U(1) \supset\left(B \backslash\left(s_{j}^{-1}(0) \cup s_{i}^{-1}(0)\right)\right) \times U(1)
\end{gathered}
$$

given by $(w, z) \longmapsto\left(w, z \frac{e_{i}(w)}{e_{j}(w)}\right)$.

We would like to make a certain element of $\operatorname{Map}^{\omega}\left(B \backslash s_{i}^{-1}(0), S L(2, \boldsymbol{R})\right)$ act on $p^{-1}\left(B \backslash s_{i}^{-1}(0)\right)$ in such a way that this action extends to an element of $\operatorname{Diff}^{\omega}(\mathcal{F})_{0}$.

Let $A_{i}: B \backslash s_{i}^{-1}(0) \longrightarrow S L(2, \boldsymbol{R})$ be the map $A_{i}=\left(\begin{array}{cc}1+\left|s_{i}\right|^{2} & 0 \\ 0 & \frac{1}{1+\left|s_{i}\right|^{2}}\end{array}\right)$ which acts on $U(1) \cong S O(2)$ identified with the oriented lines through the origin on the plane. For a neighborhood $N$ of $b \in s_{i}^{-1}(0)$, there is a real analytic coordinate

$$
N \ni w \longmapsto\left(u(w), p_{\boldsymbol{C}}\left(s_{i}(w)\right)\right) \in \boldsymbol{R}^{n-3} \times \boldsymbol{C},
$$

where $u: N \longrightarrow \boldsymbol{R}^{n-3}$ and $p_{\boldsymbol{C}}: p_{E}^{-1}(N) \longrightarrow \boldsymbol{C}=\{x+y \sqrt{-1}\}$. On this coordinate,

$$
A_{i}(w)=\left(\begin{array}{cc}
1+x^{2}+y^{2} & 0 \\
0 & \frac{1}{1+x^{2}+y^{2}}
\end{array}\right) .
$$

In order to see whether this extends to an element of $\operatorname{Diff}^{\omega}(\mathcal{F})_{0}$, we need to look at the action on another trivialization. If $N \subset B \backslash s_{j}^{-1}(0)$, we have

and

$$
x \longmapsto\left(p(x), \frac{x}{e_{j}(p(x))}\right): M \mid N \longrightarrow N \times U(1)
$$

$$
x \longmapsto\left(p(x), \frac{x}{e_{i}(p(x))}\right): M \mid\left(N \backslash s_{i}^{-1}(0)\right) \longrightarrow\left(N \backslash s_{i}^{-1}(0)\right) \times U(1) .
$$

These trivializations are related by $(w, z) \longmapsto\left(w, z \frac{e_{i}(w)}{e_{j}(w)}\right)$, which is real analytically conjugate to $(u, x, y, z) \longmapsto\left(u, x, y, R(x, y) e^{2 \pi \sqrt{-1} t_{i j}(u)} z\right)$. Here $t_{i j}(u)$ is real analytic function on $u$, for a neighborhood $D$ of $0 \in \boldsymbol{C} \cong \boldsymbol{R}^{2}, R: D \backslash\{(0,0)\} \longrightarrow S L(2 ; \boldsymbol{R})$ is the map given 
by $R(x, y)=\frac{1}{\sqrt{x^{2}+y^{2}}}\left(\begin{array}{cc}x-y \\ y & x\end{array}\right)$ in the real coordinates. Thus we need to check whether $R^{-1} A_{i} R$ is real analytic on $N$.

Lemma 3.3. - Let $R: D \backslash\{(0,0)\} \longrightarrow S L(2 ; \boldsymbol{R})$ be the map given by $R(x, y)=$ $\frac{1}{\sqrt{x^{2}+y^{2}}}\left(\begin{array}{cc}x-y \\ y & x\end{array}\right)$. Let $A=\left(\begin{array}{cc}a & 0 \\ 0 & a^{-1}\end{array}\right)$, where $a=a(x, y)$ is real analytic and $a(0,0)=1$. If $a(x, y)-1$ is divisible by $x^{2}+y^{2}$, then $R^{-1} A R$ is real analytic at $(0,0)$.

$$
\begin{aligned}
\text { Proof. - For } A & =\left(\begin{array}{cc}
a & 0 \\
0 & a^{-1}
\end{array}\right), \\
R^{-1} A R-I & =\frac{1}{x^{2}+y^{2}}\left(\begin{array}{cc}
x & y \\
-y & x
\end{array}\right)\left(\begin{array}{cc}
a & 0 \\
0 & a^{-1}
\end{array}\right)\left(\begin{array}{cc}
x-y \\
y & x
\end{array}\right)-I \\
& =\frac{1}{x^{2}+y^{2}}\left(\begin{array}{cc}
(a-1) x^{2}+\left(a^{-1}-1\right) y^{2} & \left(-a+a^{-1}\right) x y \\
\left(-a+a^{-1}\right) x y & \left(a^{-1}-1\right) x^{2}+(a-1) y^{2}
\end{array}\right) \\
& =\frac{a-1}{a\left(x^{2}+y^{2}\right)}\left(\begin{array}{cc}
a x^{2}-y^{2} & -(1+a) x y \\
-(1+a) x y & -x^{2}+a y^{2}
\end{array}\right) .
\end{aligned}
$$

Hence if $a(x, y)-1$ is divisible by $x^{2}+y^{2}$ then $R^{-1} A R$ is real analytic at $(0,0)$.

By Lemma 3.3, $A_{i}$ extends to an element of $\operatorname{Diff}^{\omega}(\mathcal{F})_{0}$. We will use $A_{i}$ to write $r_{i}{ }^{2}$ as a product of commutators. For this we look at the way to write rotations as products of commutators in $S L(2 ; \boldsymbol{R})$ and the extension question on the commutators.

\section{Lemma 3.4. - Assume that}

$$
(*)_{m} \quad a(x, y)=1+k(x, y)\left(x^{2}+y^{2}\right)^{m} \quad(k(0,0) \neq 0) \quad \text { and } \quad Y=\ell(x, y)\left(x^{2}+y^{2}\right)^{2 m}
$$

for real analytic functions $k(x, y)$ and $\ell(x, y)$ and a positive integer $m$. Then when we write the rotation $\left(\begin{array}{cc}X & -Y \\ Y & X\end{array}\right)^{2}\left(X^{2}+Y^{2}=1\right)$ as a product of two commutators as in Lemma 3.1, using the products of rotations and diagonal matrices, $W \pm Z-1$ in Lemma 3.1 are divisible by $\left(x^{2}+y^{2}\right)^{m}$.

Proof. - We have

$$
\begin{aligned}
\frac{Y}{a^{4}-1} & =\frac{\ell(x, y)\left(x^{2}+y^{2}\right)^{2 m}}{k(x, y)(a+1)\left(a^{2}+1\right)\left(x^{2}+y^{2}\right)^{m}} \\
& =\frac{\ell(x, y)}{k(x, y)(a+1)\left(a^{2}+1\right)}\left(x^{2}+y^{2}\right)^{m}
\end{aligned}
$$


and

$$
\begin{aligned}
W \pm Z-1 & =\frac{1}{\sqrt{1-\frac{\left(a^{4}+1\right)^{2} Y^{2}}{\left(a^{4}-1\right)^{2}}}}\left(\sqrt{1-Y^{2}}-\sqrt{1-\frac{\left(a^{4}+1\right)^{2} Y^{2}}{\left(a^{4}-1\right)^{2}}} \pm \frac{2 a^{2} Y}{a^{4}-1}\right) \\
& =\frac{1}{\sqrt{1-\frac{\left(a^{4}+1\right)^{2} Y^{2}}{\left(a^{4}-1\right)^{2}}}}\left(\frac{\frac{4 a^{4} Y^{2}}{\left(a^{4}-1\right)^{2}}}{\sqrt{1-Y^{2}}+\sqrt{1-\frac{\left(a^{4}+1\right)^{2} Y^{2}}{\left(a^{4}-1\right)^{2}}} \pm \frac{2 a^{2} Y}{a^{4}-1}}\right)
\end{aligned}
$$

are divisible by $\left(x^{2}+y^{2}\right)^{m}$.

Using Lemmas 3.3 and 3.4 for $m=1$, we obtain the following lemma.

Lemma 3.5. - Assume $(*)_{1}$ in Lemma 3.4. Then $\left.\underset{Y}{X} \underset{X}{-Y}\right)^{2}\left(X^{2}+Y^{2}=1\right)$ can be written as a product of two commutators which are analytic at $(x, y)=(0,0)$ after conjugated by $R$.

Proof of Proposition 3.2 continued. - For $r_{i}$, we use the trivialization

$$
M \mid\left(B \backslash s_{i}^{-1}(0)\right) \longrightarrow\left(B \backslash s_{i}^{-1}(0)\right) \times U(1) .
$$

We use the diffeomorphism of $\left(B \backslash s_{i}^{-1}(0)\right) \times U(1)$ given by $A_{i}=\left(\begin{array}{cc}1+\left|s_{i}\right|^{2} & 0 \\ 0 & \frac{1}{1+\left|s_{i}\right|^{2}}\end{array}\right)$. Then $r_{i}{ }^{2}=\left(\begin{array}{cc}X_{i} & -Y_{i} \\ Y_{i} & X_{i}\end{array}\right)^{2}$ can be written as product of 2 commutators in $\operatorname{Map}\left(B \backslash s_{i}^{-1}(0), S L(2 ; \boldsymbol{R})\right)$ by Lemma 3.1. Then for $N \subset B \backslash s_{j}^{-1}(0)$, by Lemma 3.5, the elements appearing in the commutators are real analytic on the fiber of $s_{i}^{-1}(0)$.

This completes the proof of Proposition 3.2 and the proof of Theorem 2.2 for non-trivial $U(1)$ bundles.

By using Theorem 2.2, we have the following corollary.

A closed manifold $n$-dimensional $M$ is multi $U(1)$ fibered if there exist $n$ oriented circle bundle $(U(1)$ bundle) structures with the tangent spaces of fibers spanning the tangent space $T_{x} M$ of $M$ at each point $x \in M$. For example, compact Lie groups and the 7 dimensional sphere $S^{7}$ are multi $U(1)$ fibered.

COROLlaRY 3.6. - Let $M$ be a real analytically multi $U(1)$ fibered real analytic manifold. Then the identity component $\operatorname{Diff}^{\omega}(M)_{0}$ of the group of real analytic diffeomorphisms of $M$ is a perfect group.

By using the following Proposition 3.7, Corollary 3.6 follows from Theorem 2.2.

Proposition 3.7. - Let $p_{1}: M \longrightarrow B_{1}, \ldots, p_{n}: M \longrightarrow B_{n}$ be real analytic $U(1)$ fibrations and let $\mathcal{F}_{1}, \ldots, \mathcal{F}_{n}$ be the bundle foliations. Assume that

$$
T \mathcal{F}_{1} \oplus \cdots \oplus T \mathcal{F}_{n}=T M .
$$

Let $\operatorname{Diff}^{\omega}\left(\mathcal{F}_{i}\right)_{0}$ denote the identity component of the group of real analytic diffeomorphisms of $M$ mapping each fiber of $p_{i}$ to itself. Then for an element $f \in \operatorname{Diff}^{\omega}(M)_{0}$ close to the identity, there are elements $f_{i} \in \operatorname{Diff}\left(\mathcal{F}_{i}\right)_{0}$ such that $f=f_{1} \circ \cdots \circ f_{n}$. 
Proof. - For each $U(1)$ bundle $p_{i}$, we fix the vector field $\xi_{i}$ generating the $U(1)$ action as the flow $\varphi_{t}^{(i)}(t \in \boldsymbol{R} / \boldsymbol{Z})$. We look at the real analytic mapping $F: \boldsymbol{R}^{n} \times M \longrightarrow M \times M$ given by

$$
F\left(t_{1}, \ldots, t_{n} ; x\right)=\left(\left(\varphi_{t_{1}}^{(1)} \circ \cdots \circ \varphi_{t_{n}}^{(n)}\right)(x), x\right) .
$$

By the assumption that $T M$ is the Whitney sum of $T \mathcal{F}_{i}$, the tangent map $T_{(0, x)} F: T_{0} \boldsymbol{R}^{n} \times$ $T_{x} M \longrightarrow T_{x} M \times T_{x} M$ is an isomorphism for $x \in M$. Hence by the (real analytic) inverse mapping Theorem 6.5, a neighborhood of $\{0\} \times M$ in $\boldsymbol{R}^{n} \times M$ and a neighborhood of the diagonal set of $M \times M$ are $C^{\omega}$ diffeomorphic. Thus an element $f \in \operatorname{Diff}^{\omega}(M)_{0}$ close to the identity can be written as $(f(x), x)=F\left(t_{1}(x), \ldots, t_{n}(x) ; x\right)$. For $i=n, n-1, \ldots, 1$, define $f_{i}$ by

$$
f_{i}\left(\left(f_{i+1} \circ \cdots \circ f_{n}\right)(x)\right)=\varphi_{t_{i}(x)}^{(i)} \circ \cdots \circ \varphi_{t_{n}(x)}^{(n)}(x)
$$

and we obtain the desired decomposition.

REMARK 3.8. - Such a decomposition was suggested by Sergeraert to the author in early 1980's. In the literature, we refer the readers to [9].

\section{Orbit preserving diffeomorphisms of manifolds with locally free $U(1)$ actions}

For a locally free $U(1)$ action, we can also show that any orbit preserving real analytic diffeomorphism close to the identity is homologous in $\operatorname{Diff}^{\omega}(\mathcal{F})_{0}$ to a real analytic diffeomorphism which is an orbitwise rotation. Here we say that two elements are homologous if they represent the same element in the abelianization of the group $\operatorname{Diff}^{\omega}(M)_{0}$.

Proposition 4.1. - Let $R: U(1) \times M \longrightarrow M$ be a real analytic locally free action on a real analytic manifold $M$. Let $\mathcal{F}$ denote the orbit foliation and $\operatorname{Diff}^{\omega}(\mathcal{F})_{0}$, the identity component of the group of orbit preserving real analytic diffeomorphisms. Then any orbit preserving real analytic diffeomorphism close to the identity is homologous in $\operatorname{Diff}^{\omega}(\mathcal{F})_{0}$ to a real analytic diffeomorphism which is an orbitwise rotation.

Proof. - For any point $x \in M$, let $G_{x}$ denote the isotropy subgroup at $x$ :

$$
G_{x}=\{g \in U(1) \mid g \cdot x=x\} .
$$

Then there exists a positive integer $m$ called the multiplicity of the orbit through $x$ such that $G_{x} \cong \boldsymbol{Z} / m \boldsymbol{Z}$. When $m \geqq 2$, we call the orbit through $x$ a multiple orbit of multiplicity $m$. There is an injective homomorphism $h: \boldsymbol{Z} / m \boldsymbol{Z} \longrightarrow O(n-1)(O(n-1)$ is the orthogonal group) such that a neighborhood $N$ of the orbit through $x$ is described as follows.

$$
N \cong\left(B^{n-1} \times U(1)\right) / \sim,
$$

where $B^{n-1}$ is the $(n-1)$-dimensional ball of radius $1,(w, z) \sim\left(h(k) w, e^{2 \pi \sqrt{-1} k / m} z\right)$ ( $k \in \boldsymbol{Z} / m \boldsymbol{Z}$ ) and the action of $U(1)$ on $N$ is induced from that on the $U(1)$ component.

An orbit preserving diffeomorphism $\underline{\Phi}$ of $N$ near the identity induces a unique orbit preserving diffeomorphism $\Phi(w)$ near the identity of $B^{n-1} \times U(1)$ which is $\boldsymbol{Z} / m \boldsymbol{Z}$ equivariant.

Let $\alpha \in \boldsymbol{R}$ be a Diophantine number and let

$$
\Phi(w)=R_{\lambda(w)-\alpha} \circ \Psi(w) \circ R_{\alpha} \circ \Psi(w)^{-1} \quad(w \in B)
$$

$4^{\text {e }}$ SÉRIE - TOME $42-2009-\mathrm{N}^{\mathrm{o}} 4$ 
be the expression given by Arnold's Theorem 2.1. Since $\Phi(w)$ is $\boldsymbol{Z} / m \boldsymbol{Z}$ equivariant, $\Phi(h(k) w)=R_{k / m} \circ \Phi(w) \circ R_{-k / m}$. This implies $\lambda(h(k) w)=\lambda(w)$. By substituting $h(k) w$ in the above expression and using $\lambda(h(k) w)=\lambda(w)$,

$$
\Phi(h(k) w)=R_{\lambda(w)-\alpha} \circ \Psi(h(k) w) \circ R_{\alpha} \circ \Psi(h(k) w)^{-1} .
$$

By the $\boldsymbol{Z} / m \boldsymbol{Z}$ equivariance,

$$
\begin{aligned}
\Phi(h(k) w) & =R_{k / m} \circ \Phi(w) \circ R_{-k / m} \\
& =R_{\lambda(w)-\alpha} \circ\left(R_{k / m} \circ \Psi(w) \circ R_{-k / m}\right) \circ R_{\alpha} \circ\left(R_{k / m} \circ \Psi(w) \circ R_{-k / m}\right)^{-1} .
\end{aligned}
$$

If $\Psi(w)$ is chosen such that $\int(\Psi(w)-\mathrm{id}) \mathrm{d} \theta=0$, then

$$
\int\left(\left(R_{k / m} \circ \Psi(w) \circ R_{-k / m}\right)-\mathrm{id}\right) \mathrm{d} \theta=0 .
$$

By the uniqueness of such conjugating diffeomorphisms, we see that

$$
\Psi(h(k) w)=R_{k / m} \circ \Psi(w) \circ R_{-k / m},
$$

that is, $\Psi(w)$ is also $\boldsymbol{Z} / m \boldsymbol{Z}$ equivariant. Thus $\Psi(w)$ induces an orbit preserving $C^{\omega}$ diffeomorphism $\underline{\Psi}$ of $N$ such that

$$
\underline{\Phi}=R_{\lambda(w)-\alpha} \circ \underline{\Psi} \circ R_{\alpha} \circ \underline{\Psi}^{-1},
$$

where $R_{t}$ corresponds to the action of $e^{2 \pi \sqrt{-1} t}$ and $R_{\lambda(w)}$ is an orbitwise rotation which is a $C^{\omega}$ diffeomorphism of $N$.

We take the invariant neighborhoods for the multiple orbits and regular orbits to obtain a covering of $M$. On each invariant neighborhood, we obtain the conjugating diffeomorphism $\underline{\Psi}$ and the orbitwise rotation $R_{\lambda(w)}$. They match up on the intersections of the neighborhoods and give the global diffeomorphism $\underline{\Psi}$ and the orbitwise rotation $R_{\lambda(w)}$ $(w \in M / U(1))$. Thus the proposition is proved.

\section{Orbit preserving diffeomorphisms of manifolds with semi-free $U(1)$ actions}

When we treat manifolds with special semi-free $U(1)$ actions, we need to look at orbit preserving diffeomorphisms and groups of orbitwise rotations.

Let $M=(N \times U(1)) / \sim$ be the manifold with a special semi-free $U(1)$ action. There is a map $s: N \longrightarrow M$ transverse to the orbits $\operatorname{in} \operatorname{int}(N) \times U(1)$ such that $s(\partial N)$ is the fixed point set. The normal bundle of $s(\partial N)$ is trivial and the action of $U(1)$ near $s(\partial N)$ is the product of rotation of $\boldsymbol{R}^{2}$ and trivial action in the direction of $s(\partial N)$.

Let Diff ${ }^{\omega}\left(\mathcal{O}, s(\partial N)_{k}\right)$ denote the group of the orbit preserving diffeomorphisms $f$ of $M$ such that $f-$ id is divisible by $\left(x^{2}+y^{2}\right)^{k}$, where $(x, y)$ is the coordinate normal to $s(\partial N)$ along $s(\partial N)$ where the $U(1)$ action is the rotation.

We have the following theorem for the orbit preserving diffeomorphisms of $M$ with a special semi-free $U(1)$ action.

THeOREM 5.1. - Let $M=(N \times U(1)) / \sim$ be the manifold with a special semi-free $U(1)$ action with the fixed point set $s(\partial N)$. Then $f \in \operatorname{Diff}^{\omega}\left(\mathcal{O}, s(\partial N)_{2}\right)$ can be written as product of commutators in $\operatorname{Diff}^{\omega}\left(\mathcal{O}, s(\partial N)_{0}\right)$. 
The proof of this theorem uses the following Theorem 5.3 of Arnold type for the Diophantine rotations of concentric circles on the plane. Theorem 5.3 is a spacial case of Theorem 10.1 which we prove in Section 10.

We consider the following situation. Let

$$
\Delta_{\zeta}=\left\{w \in \boldsymbol{C}^{m}|| w_{i} \mid \leq \zeta(i=1, \ldots, m)\right\} .
$$

Let $U$ be an open neighborhood of $0 \in \boldsymbol{R}^{2}$ and $\Phi: U \times\left(\Delta_{\zeta} \cap \boldsymbol{R}^{m}\right) \longrightarrow \boldsymbol{R}^{2}$ be a real analytic map such that

$$
\left(\Phi_{1}\left(x_{1}, x_{2} ; w\right)\right)^{2}+\left(\Phi_{2}\left(x_{1}, x_{2} ; w\right)\right)^{2}=x_{1}{ }^{2}+x_{2}{ }^{2} .
$$

We write $z=x_{1}+\sqrt{-1} x_{2}, \bar{z}=x_{1}-\sqrt{-1} x_{2}$ and

$$
\begin{aligned}
\Phi(z, \bar{z} ; w) & =\Phi\left(x_{1}+\sqrt{-1} x_{2}, x_{1}-\sqrt{-1} x_{2} ; w\right) \\
& =\Phi_{1}\left(x_{1}, x_{2} ; w\right)+\sqrt{-1} \Phi_{2}\left(x_{1}, x_{2} ; w\right) .
\end{aligned}
$$

Since $\Phi\left(x_{1}, x_{2} ; w\right)$ is real analytic, it is written as a convergent series in $x_{1}$ and $x_{2}$, hence $\Phi(z, \bar{z} ; w)$ is written as a convergent series in $z$ and $\bar{z}$, with analytic parameter $w$.

For the differential $D \Phi$ in the direction $\left(x_{1}, x_{2}\right)$ or $(z, \bar{z})$, assume that $D \Phi_{(0 ; w)}=$ id. For a Diophantine number $\alpha$, we consider the rotation by $2 \pi \alpha$ which is the multiplication by $e^{2 \pi \sqrt{-1} \alpha}$. The question is whether $e^{2 \pi \sqrt{-1} \alpha} \Phi$ is conjugate to the rotation by $2 \pi \sqrt{-1} \alpha$. The obvious necessary condition is that the rotation number is constant for the concentric invariant circles.

REMARK 5.2. - For each circle, this is the case by Arnold's theorem ([1]) provided that $e^{2 \pi \sqrt{-1} \alpha} \Phi$ is close to the rotation by $2 \pi \sqrt{-1} \alpha$. Since Arnold showed his theorem with real analytic parameter, it is true on $U \backslash\{0\}$. On the other hand there is the theorem by Siegel ([19], [15]) for the holomorphic diffeomorphism germ, and there also is the parametrized version.

Theorem 5.3. - There are real analytic maps $\lambda: U \times\left(\Delta_{\zeta} \cap \boldsymbol{R}^{m}\right) \longrightarrow \boldsymbol{R}(\lambda(z, \bar{z} ; w)$ depending on $(z \bar{z}, w))$ and $h: U \times\left(\Delta_{\zeta} \cap \boldsymbol{R}^{m}\right) \longrightarrow U(h(z, \bar{z} ; w) \overline{h(z, \bar{z} ; w)}=z \bar{z})$ such that

$$
e^{-2 \pi \sqrt{-1} \lambda_{w}(z)} e^{2 \pi \sqrt{-1} \alpha} \Phi(z, \bar{z} ; w)=h_{w}\left(e^{2 \pi \sqrt{-1} \alpha} h_{w}{ }^{-1}(z)\right),
$$

where $\lambda_{w}(z)=\lambda(z, \bar{z} ; w)$ and $h_{w}(z)=h(z, \bar{z} ; w)$.

We assume the above Theorem 5.3 for the Diophantine rotations of concentric circles and prove Theorem 5.1.

Proof of Theorem 5.1. - The proof goes as in the proof of Theorem 2.2 for trivial $U(1)$ bundles. We consider the Diophantine rotation along the orbits of the $U(1)$ action, that is, the action of $e^{2 \pi \sqrt{-1} \alpha} \in U(1) . f$ is thought as a real analytic family of real analytic diffeomorphisms $\Phi(w)(w \in N)$ which are near the identity. Then in a neighborhood of an interior point $w \in \operatorname{int}(N), \Phi(w)$ is written as $\Phi(w)=R_{\lambda(w)-\alpha} \circ \psi(w) \circ R_{\alpha} \circ \psi(w)^{-1}$, where $\lambda(w)$ is uniquely determined by the condition that $R_{-\lambda(w)} \Phi(w)$ has the rotation number $\alpha \bmod 1$. Since we have the section $s: N \longrightarrow M, \psi(w)$ is also determined uniquely by assuming that $\psi(w)$ fixes points of $s(N)$. For a neighborhood of a point $w \in \partial N$, by Theorem 5.3, $\Phi(w)$ is written as

$$
\Phi(w)=R_{\lambda(w)-\alpha} \circ \psi(w) \circ R_{\alpha} \circ \psi(w)^{-1},
$$


where $\lambda$ is a real analytic function on $x^{2}+y^{2}$ and $w \in N$, and $\psi(w)$ is a real analytic diffeomorphism sending each orbit to itself. The diffeomorphism $\psi(w)$ on the neighborhood of a point $w \in \partial N$ is also unique by assuming that $\psi(w)$ fixes points of $s(N)$. Thus $\psi(w)$ determines an orbit preserving real analytic diffeomorphism $\Psi$ of $M$ and $R_{\lambda(w)}$ determines a real analytic diffeomorphism $\Lambda$ of $M$ which is an orbitwise rotation. Since $f$-id is divisible by $\left(x^{2}+y^{2}\right)^{2}, \lambda$ is divisible by $\left(x^{2}+y^{2}\right)^{2}$.

We would like to use Lemma 3.1 to write $\Lambda$ as a product of commutators.

We use an orbit preserving action of the diagonal matrix $A=\left(\begin{array}{cc}a & 0 \\ 0 & a^{-1}\end{array}\right)$, where $a$ is a real analytic function of $r^{2}=x^{2}+y^{2}$.

Lemma 5.4. - Let $A=\left(\begin{array}{cc}a & 0 \\ 0 & a^{-1}\end{array}\right)$, where $a=a(x, y)$ is a real analytic function on $x^{2}+y^{2}$ and $a(0)=1$. Then the map $\widehat{A}$ preserving concentric circles given by

$$
\widehat{A}\left(\begin{array}{l}
x \\
y
\end{array}\right)=\left\|\left(\begin{array}{l}
x \\
y
\end{array}\right)\right\| A\left(\begin{array}{l}
x \\
y
\end{array}\right) /\left\|A\left(\begin{array}{l}
x \\
y
\end{array}\right)\right\|
$$

is real analytic at $(0,0)$.

Proof. - Note that

$$
\widehat{A}\left(\begin{array}{l}
x \\
y
\end{array}\right)=\frac{\sqrt{x^{2}+y^{2}}}{\sqrt{a^{2} x^{2}+a^{-2} y^{2}}}\left(\begin{array}{c}
a x \\
a^{-1} y
\end{array}\right)
$$

and

$$
\frac{\sqrt{x^{2}+y^{2}}}{\sqrt{a^{2} x^{2}+a^{-2} y^{2}}}=1 / \sqrt{1+\frac{a^{2}-1}{x^{2}+y^{2}}\left(x^{2}-\frac{y^{2}}{a^{2}}\right)} .
$$

Since $a(x, y)-1$ is divisible by $x^{2}+y^{2}, \widehat{A}$ is real analytic at $(0,0)$.

Now we can finish the proof of Theorem 5.1.

We take a real analytic function $a$ on the double $\mathbf{D} N$ of $N$ such that $a=1$ along $\partial N$, $a>1$ on $\mathbf{D} N \backslash \partial N, a$ is invariant under the involution on $\mathbf{D} N$, and the second derivative normal to $\partial N$ is nontrivial. Then we have a real analytic map $N \longrightarrow S L(2 ; \boldsymbol{R})$ given by $w \longmapsto\left(\begin{array}{cc}a(w) & 0 \\ 0 & a(w)^{-1}\end{array}\right)$. Using this diagonal matrix, by Lemma 3.1, $\lambda$ can be written as commutators in $S L(2 ; \boldsymbol{R})$ outside of $s(\partial N)$ and by Lemma 5.4 these elements used in commutators extend to $s(\partial N)$ as real analytic diffeomorphisms.

Thus we proved Theorem 5.1. 


\section{Inverse mapping theorem and its singular case}

We already used in showing Corollary 3.6, the real analytic inverse mapping theorem. To show our theorem we need an inverse mapping theorem for the real analytic maps with the Jacobian matrices being not regular.

Real analytic maps between real analytic manifolds are defined by taking the local coordinates. In fact, the definition of the real analytic manifolds relies on the real analytic inverse mapping theorem.

Before reviewing the real analytic inverse mapping theorem, we review the fundamental lemma.

Lemma 6.1. - Let $U \subset \boldsymbol{R}^{N}$ be an open set and $(T, m)$ an interval with the probability measure $m$. If $f: T \times U \longrightarrow \boldsymbol{R}$ is real analytic in $x \in U$, then $F(x)=\int_{T} f(t, x) \mathrm{d} m(t)$ is real analytic in $x$.

Proof. - For each $(t, x) \in T \times U$, there is a neighborhood $I \times V$ of $(t, x)$ and $f \mid(I \times V)$ has a complexification $(f \mid(I \times V))^{\boldsymbol{C}}: I \times V^{\boldsymbol{C}} \longrightarrow \boldsymbol{C}$. We can cover $T \times\{x\}$ by finitely many such neighborhoods and $f \mid(T \times\{x\})$ has a complexification $f^{\boldsymbol{C}}: T \times W^{\boldsymbol{C}} \longrightarrow \boldsymbol{C}$. Since $f^{\boldsymbol{C}}$ satisfies the Cauchy-Riemann equation with respect to $z \in W^{C}, F^{C}(z)=\int_{T} f^{C}(t, z) \mathrm{d} m(t)$ satisfies the Cauchy-Riemann equation as well. Hence the restriction $F(x)$ of $F^{C}$ is real analytic.

Lemma 6.2 (Hadamard Lemma). - For a real analytic function $f$ defined in a neighborhood of the origin of $\boldsymbol{R}^{N}$, there are real analytic functions $g_{1}, \ldots, g_{N}$ defined in a neighborhood of $(x, x) \in \boldsymbol{R}^{2 N}$ such that

$$
f(y)-f(x)=\sum_{i=1}^{N}\left(y_{i}-x_{i}\right) g_{i}(x, y) .
$$

Proof. - We have

$$
\begin{aligned}
f(y)-f(x) & =[f(t(y-x)+x)]_{t=0}^{t=1} \\
& =\int_{0}^{1} \sum_{i=1}^{N} \frac{\partial f}{\partial x_{i}}(t(y-x)+x)\left(y_{i}-x_{i}\right) \mathrm{d} t \\
& =\sum_{i=1}^{N}\left(y_{i}-x_{i}\right) \int_{0}^{1} \frac{\partial f}{\partial x_{i}}(t(y-x)+x) \mathrm{d} t
\end{aligned}
$$

and $\int_{0}^{1} \frac{\partial f}{\partial x_{i}}(t(y-x)+x) \mathrm{d} t$ is real analytic in $x$ and $y$.

COROLlary 6.3. - For a real analytic function $f$ defined in a neighborhood of the origin of $\boldsymbol{R}^{N}$, there are real analytic functions $h_{i j}(i, j=1, \ldots, N)$ such that

$$
f(y)-f(x)=\sum_{i=1}^{N}\left(y_{i}-x_{i}\right) \frac{\partial f}{\partial x_{i}}(x)+\sum_{i, j=1}^{N}\left(y_{i}-x_{i}\right)\left(y_{j}-x_{j}\right) h_{i j}(x, y) .
$$


We also need the following lemma given by Cartan ([3]). This is usually referred to as the closure of modules theorem (see also [7], [8]).

Lemma 6.4. - Let $f_{i}$ be a sequence of real analytic functions on $U \subset \boldsymbol{R}^{n}$. Assume that $f_{i}$ is divisible by a real analytic function $\mu(x)$ and the complexification $f_{i}{ }^{C}$ converges uniformly to the complexification of a real analytic function $f_{\infty}$ on a complexified neighborhood of $U$. Then $f_{\infty}$ is divisible by $\mu(x)$.

Now we review the real analytic inverse mapping theorem.

THEOREM 6.5 (Inverse mapping theorem). - Let $f: U \longrightarrow V$ be a real analytic map between open sets in $\boldsymbol{R}^{n}$. If the Jacobian matrix $D f_{(x)}$ at $x$ is invertible, then there are a neighborhood $W$ of $f(x)$ and a real analytic map $g: V \longrightarrow U$ such that $g(f(x))=x, g \circ f$ is the identity on $g(W)$ and $f \circ g$ is the identity on $W$.

Proof. - There are several ways to prove the real analytic inverse mapping theorem.

By the usual (differentiable) inverse mapping theorem, $f$ has a differentiable inverse map $g: W \longrightarrow U$. We need to show that $f$ is real analytic.

$f: U \longrightarrow V$ has a complexification $f^{C}: U^{C} \longrightarrow V^{C}$, where $U^{C}$ and $V^{C}$ are neighborhoods of $U$ and $V$ in $\boldsymbol{C}^{n}=\boldsymbol{R}^{n} \oplus \sqrt{-1} \boldsymbol{R}^{n}$. The Jacobian matrix $\left(D f^{\boldsymbol{C}}\right)_{(x)}$ of $f^{\boldsymbol{C}}$ is the same matrix as $D f_{(x)}=\left(\frac{\partial f_{i}}{\partial x_{j}}\right)_{i, j=1, \ldots, n}$ considered as a complex matrix. Now we look at the pull-back of $\mathrm{d} z_{i}$. Then we have

$$
\left(f^{C}\right)^{*} \mathrm{~d} z_{i}=\sum_{j=1}^{n} \frac{\partial f_{i}}{\partial x_{j}} \mathrm{~d} z_{j} .
$$

This is equivalent to the Cauchy-Riemann equation for $f_{i}^{C}=u_{i}+\sqrt{-1} v_{i}$.

By the usual (differentiable) inverse mapping theorem, there is a mapping $g^{C}: W^{C} \longrightarrow U^{C}$. Since the Jacobian matrix $D\left(g^{C}\right)_{(f(x))}$ is the inverse of $\left(D f^{\boldsymbol{C}}\right)_{(x)}$, it is the same matrix as $D g_{(f(x))}=\left(\frac{\partial g_{i}}{\partial x_{j}}\right)_{i, j=1, \ldots, n}$ considered as a complex matrix. Then we have

$$
\left(g^{C}\right)^{*} \mathrm{~d} z_{i}=\sum_{j=1}^{n} \frac{\partial g_{i}}{\partial x_{j}} \mathrm{~d} z_{j} .
$$

Thus $g_{i}^{C}$ also satisfies the Cauchy-Riemann equation. Since $g$ is the restriction of $g^{C}$ to $\boldsymbol{R}^{n}$, $g$ is real analytic.

REMARK 6.6. - There is a little more direct way to prove the inverse mapping theorem and it is more important for its generalization to the case where $D f_{(x)}=0$.

The usual proof of the differentiable inverse mapping theorem considers the fixed point of $G(y): x \longmapsto x+D f_{\left(x^{0}\right)}^{-1}(y-f(x))$. This fixed point can be obtained as the limit of the points $\left\{x^{k}\right\}_{k \geq 0}$, where $x^{0}=x^{0}, x^{1}=G(y)\left(x^{0}\right)=x^{0}+D f_{\left(x^{0}\right)}^{-1}\left(y-f\left(x^{0}\right)\right)$, $x^{k}=G(y)\left(x^{k-1}\right)(k \geq 2)$. Then

$$
G(y)\left(x^{k+1}\right)-G(y)\left(x^{k}\right)=x^{k}-x^{k-1}+D f_{\left(x^{0}\right)}^{-1}\left(f\left(x^{k-1}\right)-f\left(x^{k}\right)\right) .
$$


Since

$$
f_{i}(x)-f_{i}\left(x^{0}\right)=\sum_{j=1}^{n} \frac{\partial f_{i}}{\partial x_{j}}\left(x^{0}\right)\left(x_{j}-x_{j}^{0}\right)+\sum_{j, \ell=1}^{n} h_{i j \ell}\left(x, x^{0}\right)\left(x_{j}-x_{j}^{0}\right)\left(x_{\ell}-x_{\ell}^{0}\right)
$$

for some real analytic functions $h_{i j \ell}\left(x, x^{0}\right)$,

$$
\begin{aligned}
f_{i}\left(x^{k-1}\right)-f_{i}\left(x^{k}\right)= & \sum_{j=1}^{n} \frac{\partial f_{i}}{\partial x_{j}}\left(x^{0}\right)\left(x_{j}^{k-1}-x_{j}^{k}\right) \\
& +\sum_{j, \ell=1}^{n} h_{i j \ell}\left(x^{k-1}, x^{0}\right)\left(x_{j}^{k-1}-x_{j}^{0}\right)\left(x_{\ell}^{k-1}-x_{\ell}^{0}\right) \\
& -\sum_{j, \ell=1}^{n} h_{i j \ell}\left(x^{k}, x^{0}\right)\left(x_{j}^{k}-x_{j}^{0}\right)\left(x_{\ell}^{k}-x_{\ell}^{0}\right),
\end{aligned}
$$

and

$$
\begin{aligned}
G(y)\left(x^{k+1}\right)-G(y)\left(x^{k}\right)=D f_{\left(x^{0}\right)} & -1\left(\sum_{j, \ell=1}^{n} h_{i j \ell}\left(x^{k-1}, x^{0}\right)\left(x_{j}^{k-1}-x_{j}^{0}\right)\left(x_{\ell}^{k-1}-x_{\ell}^{0}\right)\right. \\
& \left.-\sum_{j, \ell=1}^{n} h_{i j \ell}\left(x^{k}, x^{0}\right)\left(x_{j}^{k}-x_{j}^{0}\right)\left(x_{\ell}^{k}-x_{\ell}^{0}\right)\right)_{i=1, \ldots, n} .
\end{aligned}
$$

Each component of vector given as the parenthesis $(\bullet)_{i=1, \ldots, n}$ is estimated by $\varepsilon \max _{j}\left|x_{j}^{k}-x_{j}^{k-1}\right|$ with arbitrary small $\varepsilon$ as $x^{k}$ tends to $x^{0}$. In fact,

$$
\begin{aligned}
& \sum_{j, \ell=1}^{n} h_{i j \ell}\left(x^{k-1}, x^{0}\right)\left(x_{j}^{k-1}-x_{j}^{0}\right)\left(x_{\ell}^{k-1}-x_{\ell}^{0}\right) \\
& \quad-\sum_{j, \ell=1}^{n} h_{i j \ell}\left(x^{k}, x^{0}\right)\left(x_{j}^{k}-x_{j}^{0}\right)\left(x_{\ell}^{k}-x_{\ell}^{0}\right) \\
& =\sum_{j, \ell=1}^{n}\left\{h_{i j \ell}\left(x^{k-1}, x^{0}\right)-h_{i j \ell}\left(x^{k}, x^{0}\right)\right\}\left(x_{j}^{k-1}-x_{j}^{0}\right)\left(x_{\ell}^{k-1}-x_{\ell}^{0}\right) \\
& \quad+\sum_{j, \ell=1}^{n} h_{i j \ell}\left(x^{k}, x^{0}\right)\left\{\left(x_{j}^{k-1}-x_{j}^{0}\right)\left(x_{\ell}^{k-1}-x_{\ell}^{0}\right)-\left(x_{j}^{k}-x_{j}^{0}\right)\left(x_{\ell}^{k}-x_{\ell}^{0}\right)\right\}
\end{aligned}
$$

and formulas

$$
h_{i j \ell}\left(x^{k-1}, x^{0}\right)-h_{i j \ell}\left(x^{k}, x^{0}\right)=\sum_{m=1}^{n}\left(x_{m}^{k-1}-x_{m}^{k}\right) h_{i j \ell m}^{\prime}\left(x^{k-1}, x^{k}, x^{0}\right)
$$


for some real analytic functions $h_{i j \ell m}^{\prime}\left(x^{k-1}, x^{k}, x^{0}\right)$ and

$$
\begin{aligned}
&\left(x_{j}^{k-1}-x_{j}^{0}\right)\left(x_{\ell}^{k-1}-x_{\ell}^{0}\right)-\left(x_{j}^{k}-x_{j}^{0}\right)\left(x_{\ell}^{k}-x_{\ell}^{0}\right) \\
&=\left\{\left(x_{j}^{k-1}-x_{j}^{0}\right)\left(x_{\ell}^{k-1}-x_{\ell}^{0}\right)-\left(x_{j}^{k}-x_{j}^{0}\right)\left(x_{\ell}^{k-1}-x_{\ell}^{0}\right)\right\} \\
& \quad+\left\{\left(x_{j}^{k}-x_{j}^{0}\right)\left(x_{\ell}^{k-1}-x_{\ell}^{0}\right)-\left(x_{j}^{k}-x_{j}^{0}\right)\left(x_{\ell}^{k}-x_{\ell}^{0}\right)\right\} \\
&=\left(x_{j}^{k-1}-x_{j}^{k}\right)\left(x_{\ell}^{k-1}-x_{\ell}^{0}\right)+\left(x_{j}^{k}-x_{j}^{0}\right)\left(x_{\ell}^{k-1}-x_{\ell}^{k}\right)
\end{aligned}
$$

imply that $G(y)$ is Lipschitz with small Lipschitz constant in a neighborhood of $x^{0}$.

Now we look at the same proof in a complexified neighborhood of $x^{0}$. Then $G(y)$ is holomorphic on $y$ and $x^{k}$ are also holomorphic on a neighborhood of $x^{0}$. Since this converges uniformly on this neighborhood the limit $G(y)^{\boldsymbol{C}}$ is holomorphic. Hence its real part $G(y)$ is real analytic.

Now we look at the case we are interested in. The following theorem treats the case where the Jacobian matrices of real analytic mappings are singular.

Theorem 6.7. - Let $M$ be a closed n-dimensional real analytic manifold. Let $y_{1}, \ldots, y_{n}$ be real analytic functions on $M$. Let $\left(x_{1}, \ldots, x_{n}\right)$ be a coordinate around a point $x \in M$. Let $\Delta(x)=\operatorname{det}\left(\frac{\partial y_{i}}{\partial x_{j}}\right)$ be the Jacobian. Let $f: M \longrightarrow M$ be a real analytic diffeomorphism of $M$ close to the identity such that $f-\mathrm{id}$ is divisible by $\Delta(x)^{r}(r \in \boldsymbol{Z}, r \geq 3)$. Then there are real analytic diffeomorphisms $f_{1}, \ldots, f_{n}$ such that

$$
f=f_{1} \circ \cdots \circ f_{n}
$$

where, for $k=1, \ldots, n$,

$$
\begin{aligned}
& y_{1}\left(\left(f_{k} \circ \cdots \circ f_{n}\right)(x)\right)=y_{1}(x), \ldots, y_{k-1}\left(\left(f_{k} \circ \cdots \circ f_{n}\right)(x)\right)=y_{k-1}(x), \\
& y_{k}\left(\left(f_{k} \circ \cdots \circ f_{n}\right)(x)\right)=y_{k}(f(x)), \ldots, y_{n}\left(\left(f_{k} \circ \cdots \circ f_{n}\right)(x)\right)=y_{n}(f(x)) .
\end{aligned}
$$

Moreover, $f_{i}-\mathrm{id}(i=1, \ldots, n)$ is divisible by $\Delta(x)^{r-1}$.

REMARK 6.8. - For another choice of coordinate $\left(x_{1}^{\prime}, \ldots, x_{n}^{\prime}\right)$, we have

$$
\operatorname{det}\left(\frac{\partial y_{i}}{\partial x_{k}^{\prime}}\right)=\operatorname{det}\left(\frac{\partial y_{i}}{\partial x_{j}}\right) \operatorname{det}\left(\frac{\partial x_{j}}{\partial x_{k}^{\prime}}\right)
$$

and hence the condition that $f(x)-x$ is divisible by the $r$-th power of $\Delta=\operatorname{det}\left(\frac{\partial y_{i}}{\partial x_{j}}\right)$ is independent of the choice of the coordinates.

Proof. - This follows from the following Proposition 6.9. We put

$$
u(x)=\left(y_{1}(x), \ldots, y_{k}(x), y_{k+1}(f(x)), \ldots, y_{n}(f(x))\right)-\left(y_{1}(x), \ldots, y_{n}(x)\right)
$$

and we obtain $f_{k} \circ \cdots \circ f_{n}$ as $z(x)$ in the proposition.

Proposition 6.9. - Let $U$ be an open set of $\boldsymbol{R}^{n}$ and $V$, a compact subset of $U$. Let $y=\left(y_{1}, \ldots, y_{n}\right): U \longrightarrow \boldsymbol{R}^{n}$ be a real analytic map. Put $\Delta(x)=\operatorname{det}\left(\frac{\partial y_{i}}{\partial x_{j}}(x)\right)$ and assume that $\Delta(x)$ is not the constant 0 . Let $u=\left(u_{1}, \ldots, u_{n}\right): V \longrightarrow \boldsymbol{R}^{n}$ be a sufficiently small real analytic map such that $u_{i}(x)$ is divisible by $\Delta(x)^{r}(r \in \boldsymbol{Z}, r \geq 3)$. Then there is a real analytic map $z: V \longrightarrow \boldsymbol{R}^{n}$ such that $y(z(x))=y(x)+u(x)$. Here $z(x)-x$ is divisible by $\Delta(x)^{r-1}$. 
Proof. - By Corollary 6.3, we have

$$
y_{i}(z)-y_{i}(x)=\sum_{j=1}^{n} \frac{\partial y_{i}}{\partial x_{j}}(x)\left(z_{j}-x_{j}\right)+\sum_{j, k=1}^{n} h_{i j k}(x, z)\left(z_{j}-x_{j}\right)\left(z_{k}-x_{k}\right),
$$

where $h_{i j k}$ is real analytic on a neighborhood of $(x, x)$.

If $\Delta(x)=\operatorname{det}\left(\frac{\partial y_{i}}{\partial x_{j}}(x)\right) \neq 0$ and $u(x)$ is sufficiently small, then by the inverse mapping Theorem 6.5, there uniquely exists $z(x)$ in a neighborhood of $x$.

For the point $x_{0}$ where $\Delta\left(x_{0}\right)=0$, the solution should satisfy that $z\left(x_{0}\right)=x_{0}$. We would like to know the analyticity of the map $z(x)$.

If $\Delta\left(x_{0}\right)=0, u(x)$ is divisible by $\Delta(x)^{r}$ near $x_{0}$. Put $J_{(x)}=\left(\frac{\partial y_{i}}{\partial x_{j}}(x)\right)$. We look at the following functional for real analytic map $z$ from a neighborhood of $x_{0}$ to $\boldsymbol{R}^{n}$.

$$
F(z)=z+J_{(x)}^{-1}\left(u(x)-\left\{J_{(x)}(z-x)+\left(\sum_{j, k=1}^{n} h_{i j k}(x, z)\left(z_{j}-x_{j}\right)\left(z_{k}-x_{k}\right)\right)\right\}\right)
$$

where $(\bullet)_{i}$ denotes the column vector. This functional is defined so that the fixed point $z(x)$ of $F$ is the desired map $z(x)$. Namely, if $F(z)=z$, then $u(x)=y(z)-y(x)$.

For $u(x)=\Delta(x)^{r} v(x)$, this functional is rewritten as follows. Note that $\Delta(x) J_{(x)}{ }^{-1}=$ $\left(A_{i j}(x)\right)$, where $A_{i j}(x)$ is the cofactor of $\frac{\partial y_{j}}{\partial x_{i}}(x)$.

$$
F(z)_{\ell}=x_{\ell}+\Delta(x)^{-1} \sum_{i=1}^{n} A_{\ell i}(x)\left(\Delta(x)^{r} v_{i}(x)-\sum_{j, k=1}^{n} h_{i j k}(x, z)\left(z_{j}-x_{j}\right)\left(z_{k}-x_{k}\right)\right) .
$$

We are going to find the fixed point by sequential approximation. So we put $z^{(0)}=x$, and define $z^{(m)}(m \geq 1)$ by $z^{(m)}=F\left(z^{(m-1)}\right)$. Then

$$
z_{\ell}^{(1)}-x_{\ell}=\Delta(x)^{r-1} \sum_{i=1}^{n} A_{\ell i}(x) v_{i}(x) .
$$

If $z_{i}^{(m)}(x)-x_{i}$ is divisible by $\Delta(x)^{r-1}$, then $z_{i}^{(m+1)}(x)-x_{i}$ is again divisible by $\Delta(x)^{r-1}$. For, by putting $z_{i}^{(m)}-x_{i}=\Delta(x)^{r-1} \zeta_{i}^{(m)}(x)$,

$$
\begin{aligned}
z_{\ell}^{(m+1)}-x_{\ell}= & F\left(z^{(m)}\right)_{\ell}-x_{\ell} \\
= & \Delta(x)^{r-1} \sum_{i=1}^{n} A_{\ell i}(x) v_{i}(x) \\
& -\Delta(x)^{-1} \sum_{i=1}^{n} A_{\ell i}(x) \sum_{j, k=1}^{n} h_{i j k}\left(x, z^{(m)}\right) \Delta(x)^{r-1} \zeta_{j}^{(m)} \Delta(x)^{r-1} \zeta_{k}^{(m)} \\
= & \Delta(x)^{r-1} \sum_{i=1}^{n} A_{\ell i}(x) v_{i}(x) \\
& -\Delta(x)^{2 r-3} \sum_{i=1}^{n} A_{\ell i}(x) \sum_{j, k=1}^{n} h_{i j k}\left(x, z^{(m)}\right) \zeta_{j}^{(m)} \zeta_{k}^{(m)} .
\end{aligned}
$$


Note that if $\left|\zeta_{i}^{(1)}\right| \leq \frac{K}{2}$ and $\left|\zeta_{i}^{(m)}\right| \leq K$,

$$
\left|\zeta_{\ell}^{(m+1)}\right| \leq \frac{K}{2}+K_{1}|\Delta(x)|^{r-2} K^{2}
$$

for some constant $K_{1}$. If we take $x$ close to $x_{0}$, then $|\Delta(x)| \leq \frac{1}{\sqrt{2 K_{1} K}}$ and on this neighborhood $\left|\zeta_{\ell}^{(m+1)}\right| \leq K$.

Now we would like to show that $z^{(m)}$ converges uniformly on a complexified neighborhood of $x_{0}$.

$$
\begin{aligned}
z_{\ell}^{(m+1)}-z_{\ell}^{(m)}=F\left(z^{(m)}\right)_{\ell}-F\left(z^{(m-1)}\right)_{\ell} \\
=-\Delta(x)^{-1} \sum_{i=1}^{n} A_{\ell i}(x) \sum_{j, k=1}^{n} h_{i j k}\left(x, z^{(m)}\right)\left(z_{j}^{(m)}-x_{j}\right)\left(z_{k}^{(m)}-x_{k}\right) \\
\quad+\Delta(x)^{-1} \sum_{i=1}^{n} A_{\ell i}(x) \sum_{j, k=1}^{n} h_{i j k}\left(x, z^{(m-1)}\right)\left(z_{j}^{(m-1)}-x_{j}\right)\left(z_{k}^{(m-1)}-x_{k}\right) \\
=-\Delta(x)^{-1} \sum_{i=1}^{n} A_{\ell i}(x) \sum_{j, k=1}^{n}\left\{\left(h_{i j k}\left(x, z^{(m)}\right)-h_{i j k}\left(x, z^{(m-1)}\right)\right)\left(z_{j}^{(m)}-x_{j}\right)\left(z_{k}^{(m)}-x_{k}\right)\right. \\
\left.\quad+h_{i j k}\left(x, z^{(m-1)}\right)\left(\left(z_{j}^{(m)}-x_{j}\right)\left(z_{k}^{(m)}-x_{k}\right)-\left(z_{j}^{(m-1)}-x_{j}\right)\left(z_{k}^{(m-1)}-x_{k}\right)\right)\right\} .
\end{aligned}
$$

Since

$$
h_{i j k}\left(x, z^{(m)}\right)-h_{i j k}\left(x, z^{(m-1)}\right)=\sum_{p=1}^{n}\left(z_{p}^{(m)}-z_{p}^{(m-1)}\right) h_{i j k p}^{\prime}\left(x, z^{(m)}, z^{(m-1)}\right)
$$

for some real analytic functions $h_{i j k p}^{\prime}$,

$$
\begin{aligned}
& -\Delta(x)^{-1} \sum_{i=1}^{n} A_{\ell i}(x) \sum_{j, k=1}^{n}\left(h_{i j k}\left(x, z^{(m)}\right)-h_{i j k}\left(x, z^{(m-1)}\right)\right)\left(z_{j}^{(m)}-x_{j}\right)\left(z_{k}^{(m)}-x_{k}\right) \\
& =-\Delta(x)^{2 r-3} \sum_{i=1}^{n} A_{\ell i}(x) \sum_{j, k=1}^{n} \sum_{p=1}^{n}\left(z_{p}^{(m)}-z_{p}^{(m-1)}\right) h_{i j k \ell}^{\prime}\left(x, z^{(m)}, z^{(m-1)}\right) \zeta_{j}^{(m)} \zeta_{k}^{(m)}
\end{aligned}
$$

The absolute value of the right-hand side is estimated by $|\Delta(x)|^{2 r-3} K_{2} K^{2} \max _{p} \mid z_{p}^{(m)}-$ $z_{p}^{(m-1)} \mid$ for some constant $K_{2}$. On the neighborhood where $|\Delta(x)| \leq \frac{p}{\left(4 K_{2} K^{2}\right)^{1 /(2 r-3)}}$, this is estimated by $\frac{1}{4} \max _{p}\left|z_{p}^{(m)}-z_{p}^{(m-1)}\right|$. Since

$$
\begin{aligned}
\left(z_{j}^{(m)}-x_{j}\right)\left(z_{k}^{(m)}-x_{k}\right)-\left(z_{j}^{(m-1)}-x_{j}\right)\left(z_{k}^{(m-1)}-x_{k}\right) \\
=\left(\left(z_{j}^{(m)}-x_{j}\right)\left(z_{k}^{(m)}-x_{k}\right)-\left(z_{j}^{(m-1)}-x_{j}\right)\left(z_{k}^{(m)}-x_{k}\right)\right) \\
\quad+\left(\left(z_{j}^{(m-1)}-x_{j}\right)\left(z_{k}^{(m)}-x_{k}\right)-\left(z_{j}^{(m-1)}-x_{j}\right)\left(z_{k}^{(m-1)}-x_{k}\right)\right) \\
=\left(z_{j}^{(m)}-z_{j}^{(m-1)}\right)\left(z_{k}^{(m)}-x_{k}\right)+\left(z_{j}^{(m-1)}-x_{j}\right)\left(z_{k}^{(m)}-z_{k}^{(m-1)}\right),
\end{aligned}
$$




$$
\begin{aligned}
& -\Delta(x)^{-1} \sum_{i=1}^{n} A_{\ell i}(x) \sum_{j, k=1}^{n} h_{i j k}\left(x, z^{(m-1)}\right) \\
& \cdot\left(\left(z_{j}^{(m)}-x_{j}\right)\left(z_{k}^{(m)}-x_{k}\right)-\left(z_{j}^{(m-1)}-x_{j}\right)\left(z_{k}^{(m-1)}-x_{k}\right)\right) \\
& =-\Delta(x)^{-1} \sum_{i=1}^{n} A_{\ell i}(x) \sum_{j, k=1}^{n} h_{i j k}\left(x, z^{(m-1)}\right) \\
& \cdot\left(\left(z_{j}^{(m)}-z_{j}^{(m-1)}\right)\left(z_{k}^{(m)}-x_{k}\right)+\left(z_{j}^{(m-1)}-x_{j}\right)\left(z_{k}^{(m)}-z_{k}^{(m-1)}\right)\right) \\
& =-\Delta(x)^{r-2} \sum_{i=1}^{n} A_{\ell i}(x) \sum_{j, k=1}^{n} h_{i j k}\left(x, z^{(m-1)}\right) \\
& \cdot\left(\left(z_{j}^{(m)}-z_{j}^{(m-1)}\right) \zeta_{k}^{(m)}+\left(z_{k}^{(m)}-z_{k}^{(m-1)}\right) \zeta_{j}^{(m-1)}\right) .
\end{aligned}
$$

The absolute value of the last expression is estimated by $|\Delta(x)|^{r-2} K_{3} K \max _{p} \mid z_{p}^{(m)}-$ $z_{p}^{(m-1)} \mid$. On the neighborhood where $|\Delta(x)| \leq \frac{1}{\left(4 K_{3} K\right)^{1 /(r-2)}}$, this is estimated by $\frac{1}{4} \max _{p}\left|z_{p}^{(m)}-z_{p}^{(m-1)}\right|$. Thus $z^{(m)}$ satisfies $\left|z_{\ell}^{(m)}\right| \leq \max _{p}\left|z_{p}^{(1)}\right|$, and $z^{(m)}$ converges uniformly on a complexified neighborhood of $x_{0}$. Hence the limit is holomorphic on the complexified neighborhood and $z^{(m)}$ converges to a real analytic map.

The divisibility of the limit $z(x)$ by $\Delta(x)^{r-1}$ follows from Lemma 6.4.

EXAmple 6.10. - For the unit sphere

$$
S^{n}=\left\{\left(x_{1}, \ldots, x_{n+1}\right) \in \boldsymbol{R}^{n+1} \mid x_{1}^{2}+\cdots+x_{n+1}^{2}=1\right\},
$$

we look at the map $\left(x_{1}, \ldots, x_{n}\right): S^{n} \longrightarrow \boldsymbol{R}^{n}$. Then on the coordinate $\left(x_{1}, \ldots, x_{k-1}, x_{k+1}, \ldots, x_{n}, x_{n+1}\right)$,

$$
\Delta\left(x_{1}, \ldots, x_{k-1}, x_{k+1}, \ldots, x_{n}, x_{n+1}\right)= \pm x_{n+1} / \sqrt{1-\sum_{i \neq k} x_{i}{ }^{2}} .
$$

Hence by Theorem 6.7, if a diffeomorphism $f$ of $S^{n}$ close to the identity is divisible by $\left(x_{n+1}\right)^{4}$, then $f=f_{1} \circ \cdots \circ f_{n}$, where $f_{i}$ maps an orbit of the rotation in the $x_{i} x_{n+1}$ plane to itself and $f_{i}-$ id is divisible by $\left(x_{n+1}\right)^{3}$.

The previous theorem is for one real analytic mapping. Now, we consider families of real analytic mappings. What we did in Theorem 6.7 is similar to showing that the exponential map of a Riemannian manifold is a diffeomorphism in a neighborhood of the zero of a tangent space. What we consider now in Theorem 6.12 is similar to show that the exponential map induces the diffeomorphism from a neighborhood of the zero section of the tangent bundle to a neighborhood of the diagonal set of $M \times M$.

Proposition 6.11. - Let $\xi^{(1)}, \ldots, \xi^{(n)}$ be real analytic vector fields on an open set $U$ of $\boldsymbol{R}^{n}$. Let $\varphi_{t}^{(i)}$ denote the flow generated by $\xi^{(i)}(i=1, \ldots, n)$. For a compact subset $V$ of $U$, consider the map $\Phi: \boldsymbol{R}^{n} \times V \longrightarrow U$ defined by $\Phi\left(\left(t_{1}, \ldots, t_{n}\right), x\right)=\left(\varphi_{t_{1}}^{(1)} \circ \cdots \circ \varphi_{t_{n}}^{(n)}\right)(x)$. Let $f$ be a real analytic diffeomorphism from $V$ into $U$ close to the identity and $f-$ id is divisible by $\Delta(x)^{r}$, where $\Delta(x)=\operatorname{det}\left(\xi^{(1)} \cdots \xi^{(n)}\right)$. Then there are real analytic functions 
$t_{1}(x), \ldots, t_{n}(x)$ such that $f(x)=\Phi\left(\left(t_{1}, \ldots, t_{n}\right), x\right)$. These $t_{1}(x), \ldots, t_{n}(x)$ are divisible by $\Delta^{r-1}$.

Proof. - We would like to solve

$$
f(x)-x=\Phi\left(\left(t_{1}, \ldots, t_{n}\right), x\right)-x .
$$

First note that

$$
\Phi\left(\left(t_{1}, \ldots, t_{n}\right), x\right)_{\ell}-x_{\ell}=\sum_{i=1}^{n} \xi_{\ell}^{(i)}(x) t_{i}+\sum_{i, j=1}^{n} \eta_{\ell i j}(t, x) t_{i} t_{j}
$$

for real analytic functions $\eta_{\ell i j}(t, x)$, where $t=\left(t_{1}, \ldots, t_{n}\right)$.

For the points $x$, where $\Delta(x)=\operatorname{det}\left(\xi^{(1)} \cdots \xi^{(n)}\right) \neq 0$, by the inverse mapping theorem we obtain real analytic functions $t_{1}(x), \ldots, t_{n}(x)$ such that $f(x)-x=\Phi\left(\left(t_{1}, \ldots, t_{n}\right), x\right)-x$. For the points $x$ where $\Delta(x)=0$, the solution should be $\left(t_{1}(x), \ldots, t_{n}(x)\right)=(0, \ldots, 0)$ and we would like to show the analyticity of this solution near $x$.

Put $\Xi=\left(\xi_{i}^{(j)}\right)$ and $\Delta \Xi^{-1}=\left(A_{i j}\right)$, where $A_{i j}$ is the cofactor of $\xi_{j}^{(i)}$. Now we look at the functional

$$
\begin{aligned}
F(t) & =t+\Xi^{-1}\left(f(x)-x-\left\{\Xi t+\left(\sum_{j, k=1}^{n} \eta_{i j k}(t, x) t_{j} t_{k}\right)_{i=1, \ldots, n}\right\}\right) \\
& =\Xi^{-1}\left(f(x)-x-\left(\sum_{j, k=1}^{n} \eta_{i j k}(t, x) t_{j} t_{k}\right)_{i=1, \ldots, n}\right) .
\end{aligned}
$$

Put $t^{(0)}=(0, \ldots, 0)$, and define $t^{(m)}(x)=F\left(t^{(m-1)}(x)\right)(m \geq 1)$. Since $f(x)-x$ is divisible by $\Delta(x)^{r}$, put $f(x)_{i}-x_{i}=\Delta(x)^{r} v_{i}(x)$. Then $t_{i}^{(1)}=\Delta(x)^{r-1} \sum_{j=1}^{n} A_{i j}(x) v_{j}(x)$. If $t^{(k-1)}$ is divisible by $\Delta(x)^{r-1}$, then $t^{(k)}=F\left(t^{(k-1)}\right)$ is also divisible by $\Delta(x)^{r-1}$. For, if $t_{i}^{(m)}=\Delta(x)^{r-1} \tau_{i}^{(m)}(x)$, then

$$
\begin{aligned}
& t_{\ell}^{(m+1)}=F\left(t^{(m)}\right)_{\ell} \\
& =\Delta(x)^{r-1} \sum_{i=1}^{n} A_{\ell i}(x) v_{i}(x)-\left(\Xi^{-1}\left(\sum_{j, k=1}^{n} \eta_{i j k}\left(t^{(m)}, x\right) \Delta(x)^{r-1} \tau_{j}^{(m)} \Delta(x)^{r-1} \tau_{k}^{(m)}\right)_{i}\right)_{\ell} \\
& =\Delta(x)^{r-1} \sum_{i=1}^{n} A_{\ell i}(x) v_{i}(x)-\Delta(x)^{2 r-3} \sum_{i=1}^{n} A_{\ell i}(x) \sum_{j, k=1}^{n} \eta_{i j k}\left(t^{(m)}, x\right) \tau_{j}^{(m)} \tau_{k}^{(m)} .
\end{aligned}
$$

Note that if $\left|\tau_{i}^{(1)}\right| \leq \frac{K}{2}$ and $\left|\tau_{i}^{(m)}\right| \leq K$, then

$$
\left|\tau_{\ell}^{(m+1)}\right| \leq \frac{K}{2}+K_{1} K^{2}|\Delta(x)|^{r-2}
$$

for some constant $K_{1}$. If we take $x$ close to the zeros of $\Delta$, then $|\Delta(x)| \leq \frac{1}{\left(2 K_{1} K\right)^{1 /(r-2)}}$ and we have $\left|\tau_{\ell}^{(m+1)}\right| \leq K$. 
We show that $t^{(m)}$ converges uniformly on a complexified neighborhood of the zeros of $\Delta(x)$.

$$
\begin{aligned}
t_{\ell}^{(m+1)}-t_{\ell}^{(m)}= & F\left(t^{(m)}\right)_{\ell}-F\left(t^{(m-1)}\right)_{\ell} \\
= & -\Delta(x)^{-1} \sum_{i=1}^{n} A_{\ell i}(x) \sum_{j, k=1}^{n} \eta_{i j k}\left(t^{(m)}, x\right) t_{j}^{(m)} t_{k}^{(m)} \\
& +\Delta(x)^{-1} \sum_{i=1}^{n} A_{\ell i}(x) \sum_{j, k=1}^{n} \eta_{i j k}\left(t^{(m-1)}, x\right) t_{j}^{(m-1)} t_{k}^{(m-1)} \\
= & -\Delta(x)^{-1} \sum_{i=1}^{n} A_{\ell i}(x) \sum_{j, k=1}^{n}\left\{\left(\eta_{i j k}\left(t^{(m)}, x\right)-\eta_{i j k}\left(t^{(m-1)}, x\right)\right) t_{j}^{(m)} t_{k}^{(m)}\right. \\
& \left.+\eta_{i j k}\left(t^{(m-1)}, x\right)\left(t_{j}^{(m)} t_{k}^{(m)}-t_{j}^{(m-1)} t_{k}^{(m-1)}\right)\right\} .
\end{aligned}
$$

Since

$$
\eta_{i j k}\left(t^{(m)}, x\right)-\eta_{i j k}\left(t^{(m-1)}, x\right)=\sum_{p=1}^{n}\left(t_{p}^{(m)}-t_{p}^{(m-1)}\right) \eta_{i j k p}^{\prime}\left(t^{(m)}, t^{(m-1)}, x\right)
$$

for some real analytic functions $\eta_{i j k p}^{\prime}$,

$$
\begin{aligned}
& -\Delta(x)^{-1} \sum_{i=1}^{n} A_{\ell i}(x) \sum_{j, k=1}^{n}\left(\eta_{i j k}\left(t^{(m)}, x\right)-\eta_{i j k}\left(t^{(m-1)}, x\right)\right) t_{j}^{(m)} t_{k}^{(m)} \\
& =-\Delta(x)^{2 r-3} \sum_{i=1}^{n} A_{\ell i}(x) \sum_{j, k=1}^{n} \sum_{p=1}^{n}\left(t_{p}^{(m)}-t_{p}^{(m-1)}\right) \eta_{i j k p}^{\prime}\left(t^{(m)}, t^{(m-1)}, x\right) \tau_{j}^{(m)} \tau_{k}^{(m)}
\end{aligned}
$$

The absolute value of the right-hand side is estimated by $|\Delta(x)|^{2 r-3} K_{2} K^{2} \max _{p} \mid t_{p}^{(m)}-$ $t_{p}^{(m-1)} \mid$ for some constant $K_{2}$. On the neighborhood where $|\Delta(x)| \leq \frac{p}{\left(4 K_{2} K^{2}\right)^{1 /(2 r-3)}}$, this is estimated by $\frac{1}{4} \max _{p}\left|t_{p}^{(m)}-t_{p}^{(m-1)}\right|$. Since

$$
\begin{gathered}
t_{j}^{(m)} t_{k}^{(m)}-t_{j}^{(m-1)} t_{k}^{(m-1)} \\
=\left(t_{j}^{(m)} t_{k}^{(m)}-t_{j}^{(m-1)} t_{k}^{(m)}\right)+\left(t_{j}^{(m-1)} t_{k}^{(m)}-t_{j}^{(m-1)} t_{k}^{(m-1)}\right), \\
-\Delta(x)^{-1} \sum_{i=1}^{n} A_{\ell i}(x) \sum_{j, k=1}^{n} \eta_{i j k}\left(t^{(m-1)}, x\right)\left(t_{j}^{(m)} t_{k}^{(m)}-t_{j}^{(m-1)} t_{k}^{(m-1)}\right) \\
=-\Delta(x)^{-1} \sum_{i=1}^{n} A_{\ell i}(x) \sum_{j, k=1}^{n} \eta_{i j k}\left(t^{(m-1)}, x\right) \\
\cdot\left(\left(t_{j}^{(m)}-t_{j}^{(m-1)}\right) t_{k}^{(m)}+t_{j}^{(m-1)}\left(t_{k}^{(m)}-t_{k}^{(m-1)}\right)\right) \\
=-\Delta(x)^{r-2} \sum_{i=1}^{n} A_{\ell i}(x) \sum_{j, k=1}^{n} \eta_{i j k}\left(x, z^{(m-1)}\right) \\
\cdot\left(\left(t_{j}^{(m)}-t_{j}^{(m-1)}\right) \tau_{k}^{(m)}+\left(t_{j}^{(m-1)}-t_{j}^{(m-1)}\right) \tau_{k}^{(m-1)}\right) .
\end{gathered}
$$


The absolute value of the last expression is estimated by $|\Delta(x)|^{r-2} K_{3} K \max _{p}\left|t_{p}^{(m)}-t_{p}^{(m-1)}\right|$. On the neighborhood where $|\Delta(x)| \leq \frac{1}{\left(4 K_{3} K\right)^{r-2}}$, this is estimated by $\frac{1}{4} \max _{p}\left|t_{p}^{(m)}-t_{p}^{(m-1)}\right|$. Thus $t^{(m)}$ satisfies $\left|t_{\ell}^{(m)}\right| \leq \max _{p}\left|t_{p}^{(1)}\right|$, and $t^{(m)}$ converges uniformly on a complexified neighborhood of $x_{0}$. Hence the limit is holomorphic on the complexified neighborhood and $t^{(m)}$ converges to a real analytic map.

Theorem 6.12. - Let $M$ be a closed n-dimensional real analytic manifold in $\boldsymbol{R}^{N}$. Let $\xi^{(1)}, \ldots, \xi^{(n)}$ be real analytic vector fields on $M$. Let $\varphi_{t}^{(i)}$ denote the flow generated by $\xi^{(i)}$ $(i=1, \ldots, n)$. Consider the map $\Phi: \boldsymbol{R}^{n} \times M \longrightarrow M$ defined by $\Phi\left(\left(t_{1}, \ldots, t_{n}\right), x\right)=$ $\left(\varphi_{t_{1}}^{(1)} \circ \cdots \circ \varphi_{t_{n}}^{(n)}\right)(x)$. Let $f$ be a real analytic diffeomorphism of $M$ close to the identity and $f-\mathrm{id}$ is divisible by $\Delta(x)^{r}(r \in \boldsymbol{Z}, r \geq 3)$, where $\Delta(x)=\operatorname{det}\left(\xi_{i}^{(j)}\right), \xi^{(j)}=\sum \xi_{i}^{(j)} \frac{\partial}{\partial x_{i}}$ in $a$ coordinate neighborhood $\left(U,\left(x_{1}, \ldots, x_{n}\right)\right)$ and we assume that $\Delta(x)$ is not the constant 0 . Then there are real analytic functions $t_{1}(x), \ldots, t_{n}(x)$ such that $f(x)=\Phi\left(\left(t_{1}(x), \ldots, t_{n}(x)\right), x\right)$.

REMARK 6.13. - For another coordinate neighborhood $\left(V,\left(y_{1}, \ldots, y_{n}\right)\right)$,

$$
\sum_{i=1}^{n} \xi_{i}^{(j)} \frac{\partial}{\partial x_{i}}=\sum_{i, k=1}^{n} \xi_{i}^{(j)} \frac{\partial y_{k}}{\partial x_{i}} \frac{\partial}{\partial y_{k}}
$$

and $\Delta(y)=\Delta(x) \operatorname{det}\left(\frac{\partial y_{k}}{\partial x_{i}}\right)$. Thus, the condition that $f-$ id is divisible by $\Delta(x)^{r}$ is independent of the choice of coordinate neighborhood.

Proof. - The expression $f(x)=\left(\varphi_{t_{1}}^{(1)} \circ \cdots \circ \varphi_{t_{n}}^{(n)}\right)(x)$ is unique where $\Delta(x)$ is not zero, and $\left(t_{1}, \ldots, t_{n}\right)=(0, \ldots, 0)$ on the zero set of $\Delta(x)$. By taking a coordinate neighborhood, it is real analytic on a neighborhood of a zero point of $\Delta(x)$, Hence $\left(t_{1}(x), \ldots, t_{n}(x)\right)$ is a globally defined real analytic map.

Here is the same example as before for the application of Theorem 6.12. The estimates are worse than before but it still works.

Example 6.14. - Consider the unit $n$ sphere

$$
S^{n}=\left\{\left(x_{1}, \ldots, x_{n+1}\right) \in \boldsymbol{R}^{n+1} \mid x_{1}^{2}+\cdots+x_{n+1}{ }^{2}=1\right\} .
$$

Let $\xi^{(i, j)}(1 \leq i<j \leq n+1)$ be the vector field generating the rotation in the direction of $x_{i} x_{j}$ plane, i.e.,

$$
\xi^{(i, j)}\left(x_{1}, \ldots, x_{n+1}\right)=-x_{j} \frac{\partial}{\partial x_{i}}+x_{i} \frac{\partial}{\partial x_{j}} .
$$

We look at $\xi^{(i, n+1)}(i=1, \ldots, n)$ and their time $t$ maps $\varphi_{t}^{(i, n+1)}$. Then $\Delta(x)$ with respect to the $\left(x_{1}, \ldots, x_{n}\right)$ coordinate is $\left(-x_{n+1}\right)^{n}$, and $\Delta(x)$ with respect to the $\left(x_{1}, \ldots, x_{k-1}, x_{k+1}\right.$, $\left.\ldots, x_{n}, x_{n+1}\right)$ coordinate is

$$
\pm x_{k}\left(x_{n+1}\right)^{n-1}= \pm\left(x_{n+1}\right)^{n-1} \sqrt{1-\sum_{i \neq k} x_{i}{ }^{2}} .
$$


Hence if a diffeomorphism $f$ of $S^{n}$ close to the identity is divisible by $\left(x_{n+1}\right)^{4(n-1)}$, then $f=f_{1} \circ \cdots \circ f_{n}$, where $f_{i} \circ \cdots \circ f_{n}(x)=\varphi_{t_{i}(x)}^{(i, n+1)} \circ \cdots \circ \varphi_{t_{n}(x)}^{(n, n+1)}$ and $f_{i}$ maps an orbit of $\varphi_{t}^{(i, n+1)}$ to itself. Moreover $f_{i}-$ id is divisible by $\left(x_{n+1}\right)^{3(n-1)}$.

\section{Regimentation lemma}

The key tool in the proof of the perfectness of the identity component of the group of smooth diffeomorphisms is the fragmentation lemma [2] which uses the partition of unity by the bump functions. Note that we cannot use the bump functions in the real analytic case. However, we can use the following lemma to show our Theorems 1.1 and 1.2.

Let $\mu_{1}, \ldots, \mu_{m}$ be nonnegative real analytic functions on $M$ such that $\sum_{i=1}^{m} \mu_{i}=1$. Put $S_{k}=\mu_{k}{ }^{-1}(0)$ and assume that $\bigcap_{k=1}^{m} S_{k}=\varnothing$. Put $\nu_{j}=\sum_{i=1}^{j} \mu_{i}(j=1, \ldots, m)$. Let

$$
\Phi:[0, m] \times M \longrightarrow[0,1] \times M
$$

be the map given by

$$
\Phi(t, x)=\left(\nu_{[t]}(x)+(t-[t]) \mu_{[t]+1}(x), x\right) .
$$

This map is real analytic on $[j-1, j] \times M(j=1, \ldots, m)$.

Let $\mathcal{F}$ be a foliation of $[0,1] \times M$ given by a real analytic isotopy $F:[0,1] \times M \longrightarrow M$, that is, the leaf passing through $(t, x)$ is

$$
\left\{\left(s,\left(F(s) \circ F(t)^{-1}\right)(x)\right) \mid s \in[0,1]\right\} .
$$

Theorem 7.1 (Regimentation Lemma). - If $F$ is close to the constant isotopy, then $\Phi \mid\{t\} \times M$ is transverse to $\mathcal{F}$ for $t \in[0, m]$, and $\Phi^{*} \mathcal{F}$ is real analytic isotopy on each $[j-1, j] \times M(j=1, \ldots, m)$. Thus

$$
F(0) \circ F(1)^{-1}=G_{1} \circ \cdots \circ G_{m},
$$

where $G_{j}(j=1, \ldots, m)$ are real analytic diffeomorphisms of $M$ such that $G_{j} \mid S_{j}=\operatorname{id}_{S_{j}}$. Moreover, $G_{j}-\mathrm{id}$ is divisible by $\mu_{j}$.

REMARK 7.2. - The fact that a diffeomorphism $G$ near the identity is divisible by a real analytic function $\mu$ does not depend on the choice of the coordinate neighborhood. For, let $G$ be written as $\left(g_{1}(x), \ldots, g_{n}(x)\right)$ in a coordinate $\left(x_{1}, \ldots, x_{n}\right)$ and $g_{i}\left(x_{1}, \ldots, x_{n}\right)-x_{i}$ is divisible by $\mu\left(x_{1}, \ldots, x_{n}\right)(i=1, \ldots, n)$. In another coordinate $\left(y_{1}, \ldots, y_{n}\right)$, first we have $y_{j}(z)-y_{j}(x)=\sum_{k=1}^{n}\left(z_{k}-x_{k}\right) a_{j k}(z, x)$ with real analytic functions $a_{j k}(z, x)$ by the Hadamard Lemma 6.2. Then

$$
y_{j}\left(g_{1}(x(y)), \ldots, g_{n}(x(y))\right)-y_{j}=\sum_{k=1}^{n}\left(g_{k}(x(y))-x_{k}(y)\right) a_{j k}(g(x(y)), x(y))
$$

and if $g_{i}\left(x_{1}, \ldots, x_{n}\right)-x_{i}$ is divisible by $\mu\left(x_{1}, \ldots, x_{n}\right)(i=1, \ldots, n)$, then the function $y_{j}\left(g_{1}(x(y)), \ldots, g_{n}(x(y))\right)-y_{j}$ is divisible by $\mu\left(x_{1}(y), \ldots, x_{n}(y)\right)$. 
Proof of Theorem 7.1. - This is a consequence of the real analytic inverse mapping Theorem 6.5. A real analytic foliation is defined by a family of local real analytic submersions, and the induced foliation on $[j-1, j] \times M$ is given by the composition of real analytic map $\Phi \mid[j-1, j] \times M$ and the submersion given by the isotopy, hence it is real analytic. The transversality insures that it is given by an isotopy.

To show the divisibility, we look at the induced vector field on $[j-1, j] \times M$. Let $\frac{\partial}{\partial t}+\xi(t, x)$ be the vector field on $[0,1] \times M$ defining the isotopy $F$. The map $\Phi_{j}(t, x)=$ $\left(\nu_{j-1}(x)+t \mu_{j}(x), x\right)$ induces the tangent map

$$
\left(\Phi_{j}\right)_{*}: T_{(t, x)}([0,1] \times M) \longrightarrow T_{\Phi_{j}(t, x)}([0,1] \times M)
$$

given by

$$
\begin{aligned}
& \left(\Phi_{j}\right)_{*} \frac{\partial}{\partial t}=\mu_{j}(x) \frac{\partial}{\partial t}, \\
& \left(\Phi_{j}\right)_{*} \mid\left(\{t\} \times T_{x} M\right)=\left(\left(\nu_{j-1}\right)_{*}+t\left(\mu_{j}\right)_{*}, \mathrm{id}\right) .
\end{aligned}
$$

If $\Phi_{j}{ }^{*} \mathcal{F}$ is defined by $\frac{\partial}{\partial t}+\eta_{j}(t, x)$, then we have

$$
\left(\Phi_{j}\right)_{*}\left(\frac{\partial}{\partial t}+\eta_{j}(t, x)\right)=\left(\mu_{j}(x)+\left\{\left(\nu_{j-1}\right)_{*}+t\left(\mu_{j}\right)_{*}\right\} \eta_{j}(t, x)\right) \frac{\partial}{\partial t}+\eta_{j}(t, x)
$$

and this is proportional to $\frac{\partial}{\partial t}+\xi\left(\Phi_{j}(t, x)\right)$. Hence

$$
\eta_{j}(t, x)=\left(\mu_{j}(x)+\left\{\left(\nu_{j-1}\right)_{*}+t\left(\mu_{j}\right)_{*}\right\} \eta_{j}(t, x)\right) \xi\left(\Phi_{j}(t, x)\right) .
$$

That is,

$$
\left(1-\xi\left(\Phi_{j}(t, x)\right)\left\{\left(\nu_{j-1}\right)_{*}+t\left(\mu_{j}\right)_{*}\right\}\right) \eta_{j}(t, x)=\mu_{j}(x) \xi\left(\Phi_{j}(t, x)\right) .
$$

Since $\xi(t, x)$ is small, $1-\xi\left(\Phi_{j}(t, x)\right)\left\{\left(\nu_{j-1}\right)_{*}+t\left(\mu_{j}\right)_{*}\right\}$ is invertible, and we obtain $\eta_{j}(t, x)$ as a $C^{\omega}$ time dependent vector field. Since this vector field is divisible by $\mu_{j}(x)$, by the following Proposition 7.3, for the time 1 map $G_{j}, G_{j}-$ id is divisible by $\mu_{j}(x)$.

Proposition 7.3. - Let $\xi(t, x)(t \in(-\varepsilon, \varepsilon), \varepsilon>0)$ be a real analytic time dependent vector field on an open set $U$ of $\boldsymbol{R}^{n}$. Assume that $\xi(t, x)$ is divisible by $\mu(x)$. Then for the isotopy $\varphi_{t}$ generated by $\xi(t, x), \varphi_{t}-\mathrm{id}$ is divisible by $\mu(x)$.

Proof. - The differential equation

$$
\frac{\mathrm{d} x}{\mathrm{~d} t}=\xi(t, x)=\mu(x) \eta(t, x)
$$

is solved by looking at the integral equation

$$
\varphi(t, x)=x+\int_{0}^{t} \xi(\varphi(s, x)) \mathrm{d} s .
$$

We use the method of sequential approximation. That is, first put $\varphi^{(0)}=x$ and

$$
\left.\varphi^{(k)}(t, x)=x+\int_{0}^{t} \xi\left(\varphi^{(k-1)}(s, x)\right)\right) \mathrm{d} s \quad(k \geq 1) .
$$

Then the sequence converges uniformly on $t$ and $x$ on a (small) complexified neighborhood (in $x$ ) and the limit is real analytic with respect to $x$. 
In fact, define the functional $F$ by $F(\varphi)(t, x)=x+\int_{0}^{t} \xi(s, \varphi(s, x)) \mathrm{d} s$. Assume that in a $\delta$-neighborhood $U_{\delta}\left(x^{(0)}\right)=\left\{x|| x_{i}-x^{(0)}{ }_{i} \mid \leq \delta\right\}$ of $x^{(0)}, \max _{i}\left|\frac{\partial \xi}{\partial x_{i}}\right| \leq K_{1}$. Then

$$
\left|F\left(\varphi_{1}\right)_{j}(t, x)-F\left(\varphi_{2}\right)_{j}(t, x)\right| \leq n|t| K_{1} \max _{i} \sup _{|s| \leq|t|, x \in U_{\delta}}\left|\varphi_{1 i}-\varphi_{2 i}\right| .
$$

Hence for $|t| \leq \frac{1}{2 n K_{1}}, F$ is a Lipschitz map with Lipschitz constant $\frac{1}{2}$. On the other hand, if $\max _{i}\left|\xi_{i}(s, x)\right| \leq K_{0}$ in $U_{\delta}, \max _{i}\left|\varphi_{i}^{(1)}-\varphi_{i}^{(0)}\right| \leq|t| K_{0}$. Thus if $|t| \leq \frac{\delta}{4 K_{0}}$, $\max _{i}\left|\varphi_{i}^{(1)}-\varphi_{i}^{(0)}\right| \leq \frac{\delta}{4}$. Then for $x$ with $\max _{i}\left|x_{i}-x^{(0)}{ }_{i}\right| \leq \frac{\delta}{2}, \max _{i}\left|\varphi_{i}^{(k-1)}-x^{(0)}{ }_{i}\right| \leq$ $\delta-\frac{\delta}{2 \cdot 2^{k-1}}$ implies

$$
\begin{aligned}
\max _{i}\left|\varphi_{i}^{(k)}-\varphi_{i}^{(k-1)}\right| & =\max _{i}\left|F\left(\varphi^{(k-1)}\right)_{i}-F\left(\varphi^{(k-2)}\right)_{i}\right| \\
& \leq \frac{1}{2} \max _{i}\left|\varphi_{i}^{(k-1)}-\varphi_{i}^{(k-2)}\right| \leq \frac{\delta}{4 \cdot 2^{k-1}}
\end{aligned}
$$

and hence $\max _{i}\left|\varphi_{i}^{(k)}-x^{(0)}{ }_{i}\right| \leq \delta-\frac{\delta}{2 \cdot 2^{k}}$. Then the estimates for $\varphi^{(k)}$ hold. Thus for $|t| \leq$ $\min \left\{\frac{1}{2 n K_{1}}, \frac{\delta}{4 K_{0}}\right\}, \varphi^{(k)}$ converges uniformly on $U_{\delta / 2}$. The uniform estimate holds on a complexified neighborhood and the limit $\varphi(t, x)$ is real analytic with respect to $x$ for small $|t|$. By the continuation with respect to $t$, we see that the limit $\varphi(t, x)$ is real analytic with respect to $x$ for $t \in[0,1]$.

Now since $\xi(x)=\mu(x) \eta(x)$, the sequential approximation $\varphi^{(k)}$ is given by

$$
\varphi^{(k)}(t, x)=x+\int_{0}^{t} \mu\left(\varphi^{(k-1)}(s, x)\right) \eta\left(s, \varphi^{(k-1)}(s, x)\right) \mathrm{d} s \quad(k \geq 1) .
$$

Note that

$$
\varphi^{(1)}(t, x)-x=\int_{0}^{t} \mu(x) \eta(s, x) \mathrm{d} s=\mu(x) \int_{0}^{t} \eta(s, x) \mathrm{d} s
$$

is divisible by $\mu(x)$. If $\varphi^{(k-1)}(t, x)-x$ is divisible by $\mu(x)$ and say $\varphi^{(k-1)}(t, x)-x=$ $\mu(x) \eta^{(k-1)}(t, x)$, then

$$
\varphi^{(k)}(t, x)-x=\int_{0}^{t} \mu\left(\varphi^{(k-1)}(s, x)\right) \eta^{(k-1)}\left(s, \varphi^{(k-1)}(s, x)\right) \mathrm{d} s
$$

and by the Hadamard Lemma 6.2, there are real analytic functions $\alpha_{j}(x, y)$ such that

$$
\begin{aligned}
\mu(y)-\mu(x)=\sum_{j=1}^{n}\left(y_{j}-x_{j}\right) \alpha_{j}(x, y) \text { and } & \\
\mu\left(\varphi^{(k-1)}(s, x)\right) & =\mu\left(x+\mu(x) \eta^{(k-1)}(s, x)\right) \\
& =\mu(x)+\sum_{j=1}^{n} \mu(x) \eta_{j}^{(k-1)}(s, x) \alpha_{j}\left(x, x+\mu(x) \eta_{j}^{(k-1)}(s, x)\right)
\end{aligned}
$$


is divisible by $\mu(x)$. Hence $\varphi^{(k)}(t, x)-x$ is divisible by $\mu(x)$. Since the limit of a sequence of real analytic functions divisible by $\mu(x)$ is divisible by $\mu(x)$ by Lemma $6.4, \varphi_{t}-$ id is divisible by $\mu(x)$.

Here is an example of application of the regimentation lemma.

ExAmple 7.4. - Let $S^{n}=\left\{x \in \boldsymbol{R}^{n+1} \mid\|x\|=1\right\}$. We have the functions

$$
\mu_{i}=x_{i}{ }^{4} / \sum_{j} x_{j}{ }^{4}: S^{n} \longrightarrow \boldsymbol{R}
$$

such that $\sum_{i=1}^{n+1} \mu_{i}=1$. We think about the decomposition of real analytic diffeomorphisms of $S^{n}$ into regimented diffeomorphisms by Lemma 7.1. Then for a diffeomorphism $f: S^{n} \longrightarrow S^{n}$ close to the identity, $f=f^{(1)} \circ \cdots \circ f^{(n+1)}$, where $f^{(i)}-$ id is divisible by $\mu_{i}$, i.e., 4-flat along $\left\{x \in S^{n} \mid x_{i}=0\right\}(i=1, \ldots, n+1)$.

For a diffeomorphism $f: S^{n} \longrightarrow S^{n}$ close to the identity, as in Example 7.4, $f$ is decomposed into a composition of diffeomorphisms which are flat along coordinate hyperplanes. Then as in Example 6.10, such flat diffeomorphisms are decomposed into a composition of orbit preserving diffeomorphisms. Then by Theorem 5.1, these orbit preserving diffeomorphisms can be written as product of commutators of orbit preserving diffeomorphisms. Hence we obtain the following corollary.

COROllary 7.5. - The identity component $\operatorname{Diff}^{\omega}\left(S^{n}\right)_{0}$ is a perfect group.

\section{Proof of main theorems}

Proposition 8.1. - Let $M$ be a compact real analytic manifold with nontrivial $U(1)$ action. Let $\xi$ be the generating vector field for the $U(1)$ action. Then there are finitely many real analytic diffeomorphisms $f_{i}, i=1, \ldots, N\left(f_{1}=\mathrm{id}\right)$ of $M$ such that, for any point $x \in M$, there is a subset $\left\{i_{1}, \ldots, i_{n}\right\} \subset\{1, \ldots, N\}$ with $\left(f_{i_{1}}\right)_{*} \xi, \ldots,\left(f_{i_{n}}\right)_{*} \xi$ spanning $T_{x} M$.

Proof. - For each point $x \in M$, there is a point $y$ close to $x$ where $\xi(y) \neq 0$. Then there are $C^{1}$ diffeomorphisms $g_{1}, \ldots, g_{n}$ of $M$ such that $\left(g_{1}\right)_{*} \xi, \ldots,\left(g_{n}\right)_{*} \xi$ span $T_{x} M$. Now we take real analytic approximations $f_{1}^{x}, \ldots, f_{n}^{x}$ of them. Then $\left(f_{1}^{x}\right)_{*} \xi, \ldots,\left(f_{n}^{x}\right)_{*} \xi$ span $T_{x} M$ and these span $T_{x^{\prime}} M$ for $x^{\prime}$ in a neighborhood $U_{x}$ of $x$. We cover $M$ by these $U_{x}$ and take a finite subcover and obtain $f_{1}, \ldots, f_{N}$.

Proof of main Theorems 1.1 and 1.2. - Let $M$ be a $U(1)$ fibered manifold or a manifold admitting a special semi-free $U(1)$ action.

We put a real analytic Riemannian metric on $M$. We use Proposition 8.1 and obtain $U(1)$ actions generated by $\xi_{1}, \ldots, \xi_{N}$ with the following property. For each choice $\kappa$ of $n$ vector fields among $\left\{\xi_{1}, \ldots, \xi_{N}\right\}$, we have the determinant $\Delta_{\kappa}=\operatorname{det}\left(\xi_{k_{i} j}\right)$ with respect to an orthonormal frame $\frac{\partial}{\partial x_{j}}$ at each point, where $\kappa=\left\{\xi_{k_{1}}, \ldots, \xi_{k_{n}}\right\}, n=\operatorname{dim}(M)$, and $\xi_{k_{i}}=\sum_{j=1}^{n} \xi_{k_{i} j} \frac{\partial}{\partial x_{j}}$. By Remark 6.13, $\Delta_{\kappa}$ does not depend on the choice of the orthonormal frame and $\Delta_{\kappa}$ is a real analytic function on $M$. 
Then $M$ is covered by the open sets of the form $M \backslash\left\{\Delta_{\kappa}=0\right\}$. By the regimentation Lemma 7.1, any real analytic diffeomorphism $f$ close to the identity is decomposed into real analytic diffeomorphisms $f_{\kappa}$ such that $f_{\kappa}-$ id is divisible by $\left(\Delta_{\kappa}\right)^{4}$. Then by the inverse function Theorem 6.12 for multi-vector fields, $f_{\kappa}$ can be written as a composition of orbit preserving diffeomorphisms. For a special semi-free $U(1)$ action, $\Delta_{\kappa}=0$ along the fixed point set $s(\partial N)$ and these orbit preserving diffeomorphisms satisfy the assumption of Theorem 5.1.

Then by Theorems 2.2 and 5.1, orbit preserving diffeomorphisms can be written as product of commutators in the group of orbit preserving diffeomorphisms. Thus Theorems 1.1 and 1.2 are proved.

\section{Real analytic diffeomorphisms of $\mathbf{2}$ and $\mathbf{3}$ dimensional manifolds with $U(1)$ actions}

In this section, we show that $\operatorname{Diff}^{\omega}(M)_{0}$ for 2 and 3 dimensional manifolds $M$ with nontrivial $U(1)$ actions are perfect.

First we note that, by the proof of main theorems, if $M$ admits a nontrivial $U(1)$ action, any element of $\operatorname{Diff}^{\omega}(M)_{0}$ is homologous to a diffeomorphism which is an orbitwise rotation. Here we say that two elements are homologous if they represent the same element in the abelianization of the group $\operatorname{Diff}^{\omega}(M)_{0}$.

Proposition 9.1. - If $M$ admits a nontrivial $U(1)$ action, any real analytic diffeomorphism isotopic to the identity is homologous to a diffeomorphism which is an orbitwise rotation.

Proof. - We note first that by the proof of main theorems, if $M$ admits a nontrivial $U(1)$ action, using Proposition 8.1 of perturbation, the regimentation Lemma 7.1 and the inverse function Theorem 6.12 for multi-vector fields, any real analytic diffeomorphism $f$ close to the identity can be written as a composition of orbit preserving diffeomorphisms. Note that these orbit preserving diffeomorphisms are conjugate to orbit preserving diffeomorphisms of the original $U(1)$ action.

If $M$ admits a locally free $U(1)$ action, Proposition 4.1 implies that orbit preserving diffeomorphisms close to the identity are homologous to diffeomorphisms which are orbitwise rotations. In general, the argument of the proof of Proposition 4.1 together with Theorem 5.3 (and its generalization Theorem 10.1) implies that orbit preserving diffeomorphisms, obtained by the regimentation Lemma 7.1 and the inverse function Theorem 6.12 for multivector fields, are homologous to diffeomorphisms which are orbitwise rotations. Here, note that Theorem 10.1 in Section 10 gives

$$
H_{w}(z)=\left(z_{1} e^{2 \pi \sqrt{-1} m_{1} k_{w}(z)}, \ldots, z_{n} e^{2 \pi \sqrt{-1} m_{n} k_{w}(z)}\right)
$$

such that $\Phi_{w}(z)=\left(R_{\lambda_{w}(z)} \circ R_{-\alpha} \circ H_{w} \circ R_{\alpha} \circ H_{w}^{-1}\right)(z)$. This $H_{w}$ can be replaced by $H_{w} \circ R_{-\beta_{w}(z)}$, where $\beta_{w}(z)$ is real analytic function constant along the orbit. Put $\beta_{w}(z)=\frac{1}{2 \pi} \int k_{w}(z) \mathrm{d} \theta$, then

$$
\left.\left(H_{w} \circ R_{-\beta_{w}(z)}\right)(z)=\left(z_{1} e^{\left.2 \pi \sqrt{-1} m_{1} k_{w}\left(z e^{-2 \pi \sqrt{-1} \beta_{w}(z)}\right)\right)}, \ldots, z_{n} e^{2 \pi \sqrt{-1} m_{n} k_{w}\left(z e^{-2 \pi \sqrt{-1}} \beta_{w}(z)\right.}\right)\right)
$$

$4^{\mathrm{e}}$ SÉRIE - TOME $42-2009-\mathrm{N}^{\mathrm{o}} 4$ 
and $k_{w}\left(z e^{-2 \pi \sqrt{-1} \beta_{w}(z)}\right)$ satisfies

$$
\begin{aligned}
\int_{S^{1}} k_{w} & \left(z e^{-2 \pi \sqrt{-1} \beta_{w}(z)}\right) \mathrm{d} \theta \\
& =\int_{S^{1}}\left(k_{w}\left(z e^{-2 \pi \sqrt{-1} \beta_{w}(z)}\right)-k_{w}(z)+k_{w}(z)\right)(\theta) \mathrm{d} \theta \\
& =-\int_{S^{1}}\left(k_{w}{ }^{-1}(z)+\beta_{w}(z)-k_{w}{ }^{-1}(z)\right)(\theta) \mathrm{d} \theta+\int_{S^{1}} k_{w}(z)(\theta) \mathrm{d} \theta=0 .
\end{aligned}
$$

Thus the conjugating real analytic diffeomorphisms match up on $M$ as in the proof of Proposition 4.1 .

Since the product of orbitwise rotations is an orbitwise rotation, we showed that any real analytic diffeomorphism close to the identity is homologous to an orbitwise rotation. Since any element of $\operatorname{Diff}^{\omega}(M)_{0}$ is a composition of diffeomorphism close to the identity, it is homologous to an orbitwise rotation.

Now we prove Theorem 1.3. This is done by showing following Propositions 9.2, 9.3 and Theorem 9.4.

If the dimension of $M$ is 2 , then $M$ with nontrivial $U(1)$ action is diffeomorphic to the torus $T^{2}$, the sphere $S^{2}$, the Klein bottle $K^{2}$ or the real projective plane $\boldsymbol{R} P^{2}$. For the torus $T^{2}, \operatorname{Diff}^{\omega}\left(T^{2}\right)_{0}$ is simple by the result of Herman ([11]). For the sphere $S^{2}$, our Theorem 1.2 says that $\operatorname{Diff}^{\omega}\left(S^{2}\right)_{0}$ is perfect.

Proposition 9.2. - $\operatorname{Diff}^{\omega}\left(K^{2}\right)_{0}$ is perfect.

Proof. - For the Klein bottle $K^{2}$, we have a locally free $U(1)$ action with 2 multiple orbits of multiplicity 2. By Proposition 9.1, any element of Diff ${ }^{\omega}\left(K^{2}\right)_{0}$ is homologous to a diffeomorphism which is an orbitwise rotation. $K^{2} / U(1)$ is an interval. We can take a circle transverse to the $U(1)$ orbits which is $\boldsymbol{Z} / 2 \boldsymbol{Z}$ equivariant, where $\boldsymbol{Z} / 2 \boldsymbol{Z} \subset U(1)$. Using this circle, we can make $S L(2 ; \boldsymbol{R})$ act on the regular orbits so that the induced action on the 2 multiple orbits is the action of $P S L(2 ; \boldsymbol{R})$. Using this we can write orbitwise rotations close to the identity as a product of commutators of orbit preserving diffeomorphisms. Thus $\operatorname{Diff}^{\omega}\left(K^{2}\right)_{0}$ is perfect.

Proposition 9.3. - $\operatorname{Diff}^{\omega}\left(\boldsymbol{R} P^{2}\right)_{0}$ is perfect.

Proof. - For the real projective plane $\boldsymbol{R} P^{2}$, there is a $U(1)$ action with 1 fixed point and 1 multiple orbit of multiplicity 2. By Proposition 9.1, any element of $\operatorname{Diff}^{\omega}\left(\boldsymbol{R} P^{2}\right)_{0}$ is homologous to a diffeomorphism which is an orbitwise rotation. We can arrange so that this orbitwise rotation satisfies that $f-$ id is divisible by $\left(x^{2}+y^{2}\right)^{2}$ at the fixed point. We have the action of Lemma 5.4 which extends to the multiple orbit of multiplicity 2 , and using it we can write orbitwise rotations close to the identity as a product of commutators of orbit preserving diffeomorphisms. Thus $\operatorname{Diff}^{\omega}\left(\boldsymbol{R} P^{2}\right)_{0}$ is perfect. 
All other closed 2-dimensional manifolds are hyperbolic and do not admit nontrivial $U(1)$ action. We do not know the abelianization of $\operatorname{Diff}^{\omega}(M)_{0}$ for a hyperbolic closed 2-dimensional manifold $M$.

Now we consider a closed 3-dimensional manifold $M^{3}$ admitting a nontrivial $U(1)$ action. Those manifolds with $U(1)$ actions are classified by Raymond ([17]) and Orlik-Raymond ([16]).

In the rest of this section, we show the following theorem.

THEOREM 9.4. - Let $M^{3}$ be a closed oriented 3-dimensional real analytic manifold admitting a nontrivial $U(1)$ action. Then the identity component $\operatorname{Diff}^{\omega}\left(M^{3}\right)_{0}$ of the group of real analytic diffeomorphisms of $M$ is a perfect group.

First we assume that the action is locally free. If there are no multiple orbits, then the theorem follows from our Theorem 1.1. Hence we assume that there are multiple orbits. Then the quotient space $M^{3} / U(1)$ is a 2-dimensional orbifold with boundary which corresponds to the multiple orbit of multiplicity 2 with the homomorphism $\boldsymbol{Z} / 2 \boldsymbol{Z} \longrightarrow O(2)$ sending the generator to an orientation reversing map. Let $S_{1}^{1}, \ldots, S_{n}^{1}$ denote the boundary components.

Other than orbits corresponding to the boundary, there are finitely many multiple orbits $O_{1}, \ldots, O_{\ell}$, where the isotropy subgroups are nontrivial. Let $m_{1}, \ldots, m_{\ell}$ be the multiplicity of $O_{1}, \ldots, O_{\ell}$. Let $k$ be the least common multiple of $m_{1}, \ldots, m_{\ell}$ if the boundary is empty, and $k$ be the least common multiple of $m_{1}, \ldots, m_{\ell}$ and 2 if the boundary is not empty.

For the multiple orbit $O_{i}$, we have a neighborhood $N_{i} \cong\left(B^{2} \times U(1)\right) / \sim$, where

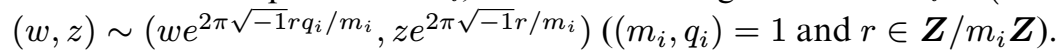

For the boundary component $S_{i}^{1}$ of $M / U(1)$, we have a neighborhood $N_{i}^{\prime} \cong$ $\left([-1,1] \times S_{i}^{1} \times U(1)\right) / \sim$ of the component of multiple orbits, where $(u, v, z) \sim(-u, v,-z)$.

For each $N_{i}$, we take $k$ sections $B^{2} \times\left\{e^{2 \pi \sqrt{-1} j / k}\right\}(j=0, \ldots, k-1)$ in $B^{2} \times U(1)$, and then this gives $k / m_{i}$ disks in $N_{i}$ transverse to the multiple orbit $O_{i}$.

For each $N_{i}^{\prime}$, we can take a family of curves $\gamma_{i}$ on $S_{i}^{1} \times U(1)$ such that $\gamma_{i}$ intersects $\{x\} \times U(1)\left(x \in S_{i}^{1}\right)$ in $k$ points and is invariant under the translation by $e^{2 \pi \sqrt{-1} / k}$ in the $U(1)$ direction. We take a family of annuli $[-1,1] \times \gamma_{i}$ in $[-1,1] \times S_{i}^{1} \times U(1)$. Then, for each $N_{i}^{\prime}$, this gives a family of annuli transverse to each multiple orbit in $\left(\{0\} \times S_{i}^{1} \times U(1)\right) / \sim$ at $k / 2$ points.

The quotient space $\underline{\Sigma}=M / U(1)$ is an orbifold which is topologically a connected surface of genus $g$ with $\ell$ marked points and possibly with the boundary. Then the projection $M \longrightarrow \underline{\Sigma}=M / U(1)$ has a multi-section on each $N_{i} / U(1)$ and $N_{i}^{\prime} / U(1)$. We try to extend the multi-section over $\underline{\Sigma}$. Put $g^{\prime}=2 g$ if the surface $\underline{\Sigma}$ is orientable, and put $g^{\prime}=g$ if the surface $\underline{\Sigma}$ is nonorientable.

If the boundary is empty, we choose arcs $A_{1}, \ldots, A_{\ell-1}, B_{1}, \ldots, B_{g^{\prime}}$ on $\underline{\Sigma}=M / U(1)$ connecting $N_{i} / U(1)$ so that the complement of $\bigcup_{i=1}^{\ell}\left(N_{i} / U(1)\right) \cup \bigcup_{i=1}^{\ell-1} A_{i} \cup \bigcup_{i=1}^{g^{\prime}} B_{i}$ is simply connected. We extend the multi-section over these arcs, and then we have a multi-section along the boundary of the simply connected region. We have the obstruction to extend this multi-section to the simply connected region, which is the Euler class. 
If there are multiple orbits $O_{i}$ and the boundary is not empty, we also choose arcs $A_{1}, \ldots, A_{\ell-1}, B_{1}, \ldots, B_{g^{\prime}}$ on $\underline{\Sigma}=M / U(1)$ connecting $N_{i} / U(1)$ and arcs $C_{i}$ connecting $A_{1}$ to $N_{i}^{\prime} / U(1)(i=2, \ldots, n)$ so that the complement of $\bigcup_{i=1}^{\ell}\left(N_{i} / U(1)\right) \cup \bigcup_{i=2}^{n}\left(N_{i}^{\prime} / U(1)\right) \cup$ $\bigcup_{i=1}^{\ell-1} A_{i} \cup \bigcup_{i=1}^{g^{\prime}} B_{i} \cup \bigcup_{i=2}^{n} C_{i}$ is an annulus with a boundary component being $S_{1}^{1}$. We choose a multi-section on each $N_{i} / U(1)(i=2, \ldots, \ell)$ and on each $N_{i}^{\prime} / U(1)(i=2, \ldots, n)$. We extend the multi-section over these arcs, and then we can extend it to the whole $\underline{\Sigma}=M / U(1)$.

If there are no multiple orbits other than the boundary, we take $\operatorname{arcs} C_{i}(i=2, \ldots, n-1)$ connecting $N_{i}^{\prime} / U(1)(i=2, \ldots, n)$ so that the complement of $\bigcup_{i=2}^{n}\left(N_{i}^{\prime} / U(1)\right) \cup \bigcup_{i=2}^{n-1} C_{i}$ is an annulus with a boundary component being $S_{1}^{1}$. We choose a multi-section on each $N_{i} / U(1)$ $(i=2, \ldots, \ell)$, we extend the multi-section over these arcs, and then we can extend it to the whole $\underline{\Sigma}=M / U(1)$. If the boundary is the circle $S_{1}^{1}$, then $\underline{\Sigma}$ is the disk or the Möbius band, and we have a multi-section $(k=2)$.

Now we need to know that we can choose the multi-section real-analytically and invariant under the action of $\boldsymbol{Z} / k \boldsymbol{Z}$, possibly outside of several regular orbits if the boundary is empty.

Consider $\underline{M}=M /(\boldsymbol{Z} / k \boldsymbol{Z})$. For each multiple orbit $O_{i}$,

$$
N_{i} /(\boldsymbol{Z} / k \boldsymbol{Z}) \cong\left(B^{2} /\left(\boldsymbol{Z} / m_{i} \boldsymbol{Z}\right)\right) \times(U(1) /(\boldsymbol{Z} / k \boldsymbol{Z})),
$$

where $B^{2} /\left(\boldsymbol{Z} / m_{i} \boldsymbol{Z}\right)$ is a cone of angle $2 \pi / m_{i}$. For each boundary component $S_{i}^{1}$,

$$
N_{i}^{\prime} /(\boldsymbol{Z} / k \boldsymbol{Z}) \cong[0,1] \times S^{1} \times(U(1) /(\boldsymbol{Z} / k \boldsymbol{Z})) .
$$

Then the action of $U(1) /(\boldsymbol{Z} / k \boldsymbol{Z})$ is free on $\underline{M}$. The multi-section we took corresponds to a section of this $U(1) /(\boldsymbol{Z} / k \boldsymbol{Z})$ bundle $\underline{M} \longrightarrow \underline{\Sigma}$.

For the cases where there is a multi-section for $M \longrightarrow \underline{\Sigma}$, we approximate this section for $\underline{M} \longrightarrow \underline{\Sigma}$, by a real analytic section and the inverse image of it under $M \longrightarrow \underline{M}$ is the desired real analytic multi-section for $M \longrightarrow \underline{\Sigma}$. Note that, for the real analyticity around the cone points and the boundary, we understand as follows. A function $f$ on $B^{2} /\left(\boldsymbol{Z} / m_{i} \boldsymbol{Z}\right)$ is real analytic if $f$ is induced from a real analytic function on $B^{2}$ invariant under the action of $\boldsymbol{Z} / m_{i} \boldsymbol{Z}$. A function $f$ on $[0,1] \times S_{i}^{1}$ is real analytic if it is induced from a real analytic function on $[-1,1] \times S_{i}^{1}$ invariant under the map $(u, v) \longmapsto(-u, v)$.

If there are obstructions to construct multi-sections, we proceed as follows. Let $\underline{E}$ be the $C$ bundle over $\underline{\Sigma}=M / U(1)$ associated to $\underline{M} \longrightarrow \underline{\Sigma}$. We consider real analytic sections of $\underline{E} \longrightarrow \underline{\Sigma}$.

We divide into two cases according to the uniformizability of $\underline{\Sigma}=M / U(1)$. We treat the case where $\underline{\Sigma}=M / U(1)$ is not uniformizable in a different way later.

If $\underline{\Sigma}=M / U(1)$ is uniformizable, there is a finite branched cover

$$
\Sigma \longrightarrow \Sigma / F \cong \underline{\Sigma}=M / U(1),
$$

where $F$ is a finite group acting on the real analytic surface $\Sigma$. This map $\Sigma \longrightarrow \underline{\Sigma}$ is covered by the $C$ bundle map $E_{\Sigma} \longrightarrow \underline{E}$. First, take a smooth section which is not zero on $B^{2} /\left(\boldsymbol{Z} / m_{i} \boldsymbol{Z}\right)(i=1, \ldots, \ell)$ and is transverse to the zero section of $\underline{E} \longrightarrow \underline{\Sigma}$. This gives an $F$ equivariant smooth section of $E_{\Sigma} \longrightarrow \Sigma$. Let $s$ be a real analytic section of $E_{\Sigma} \longrightarrow \Sigma$ 
which approximates the smooth equivariant section. Then we take the average by the action of $F$. That is, let $\sigma=\frac{1}{|F|} \sum_{\gamma \in F} \gamma \cdot s \circ \gamma^{-1}$. Then $\sigma$ is equivariant and still not zero on the preimages of $B^{2} /\left(\boldsymbol{Z} / m_{i} \boldsymbol{Z}\right)$. The section $\sigma$ is transverse to the zero section because the maps $\gamma \cdot s \circ \gamma^{-1}(\gamma \in F)$ are close to each other, each zero of $\sigma$ is near a zero of $\gamma \cdot s \circ \gamma^{-1}(\gamma \in F)$ and the tangent maps of $\gamma \cdot s \circ \gamma^{-1}$ near the zero of $\sigma$ are close to each other. Thus this $\sigma$ induces a real analytic section $\underline{\sigma}$ of $\underline{E} \longrightarrow \underline{\Sigma}$.

Outside the zeros of $\underline{\sigma}, \underline{\sigma}$ gives a real analytic section of $\underline{M} \longrightarrow \underline{\Sigma}$. Now we take the inverse image $\widehat{\sigma}$ of $\underline{\sigma}(\underline{\Sigma} \backslash \operatorname{Zero}(\underline{\sigma}))$ under $M \longrightarrow \underline{M}$. This $\widehat{\sigma}$ gives the desired multi-section outside the zeros of $\underline{\sigma}$.

Now we have necessary real analytic multi-sections $\widehat{\sigma}$ when the boundary of $\underline{\Sigma}$ is not empty or $\underline{\Sigma}$ is uniformizable.

Let $\widetilde{S L}(2 ; \boldsymbol{R})$ denote the $k$ fold covering group of $S L(2 ; \boldsymbol{R})$. Using this section, we make the diagonal matrices of $\widetilde{S L^{k}}(2 ; \boldsymbol{R})$ act along the orbits.

If there is a real analytic multi-section $\underline{\sigma}$ over $\underline{\Sigma}$, then we make $\widetilde{S L^{k}}(2 ; \boldsymbol{R})$ act along the orbits. By Lemma 3.1, orbitwise rotations close to the identity can be written as a product of commutators of orbit preserving diffeomorphisms, and the theorem of this case is shown.

If Zero $(\underline{\sigma})$ is not empty and there is a multi-section only over $\underline{\Sigma} \backslash \operatorname{Zero}(\underline{\sigma})$, we need a multisection version of Lemma 3.3, which is proved by looking at the $k$ fold covering along the fiber and lift the maps appearing in the proof of Lemma 3.3 to the $k$ fold covering.

Lemma 9.5. - Let $R: D \backslash\{(0,0)\} \longrightarrow S L(2 ; \boldsymbol{R})$ be the map given in Lemma 3.3. Let $A=\left(\begin{array}{cc}a & 0 \\ 0 & a^{-1}\end{array}\right)$, where $a=a(x, y)$ is real analytic and $a(0,0)=1$. If $a(x, y)-1$ is divisible by $x^{2}+y^{2}$, then $R^{-1}$ AR lifts to a real analytic map $\widehat{A}_{k}: D \longrightarrow \widetilde{S L}{ }^{k}(2 ; \boldsymbol{R})$ such that $\widehat{A}_{k}(0,0)=$ id.

We note that Lemma 3.4 can be applied to the lifts of the actions of the rotations and diagonal matrices and this together with Lemma 9.5 implies the following lemma.

Lemma 9.6. - The orbitwise action $\operatorname{Map}\left(D \backslash\{0\}, \widetilde{S L}{ }^{k}(2 ; \boldsymbol{R})\right)$ which is the lift of the action given in Lemma 3.5 extends real analytically to the fiber on 0.

By using real analytic sections $\underline{\sigma}$ whose zero sets are disjoint, we can write an orbitwise rotation as a product of orbitwise rotations such that the condition $(*)_{1}$ of Lemma 3.5 is satisfied as in the proof of Proposition 3.2. Then by Lemma 3.1, using the action of elements of $\widetilde{S L^{k}}(2 ; \boldsymbol{R})$, we can write an orbitwise rotation close to the identity, as a product of two commutators in $\operatorname{Map}^{\omega}\left(\underline{\Sigma} \backslash \operatorname{Zero}(\underline{\sigma}), \widetilde{S L^{k}}(2 ; \boldsymbol{R})\right)_{0}$. Then by Lemma 9.6, the diffeomorphisms appearing in the commutators are real analytic on the fibers of $\operatorname{Zero}(\underline{\sigma})$.

If $M / U(1)$ is not uniformizable, $\underline{\Sigma}=M / U(1)$ is the 2-dimensional sphere $S^{2}$ with 1 or 2 cone points. In this case, $M$ is a lens space $L(p, q)$ for coprime integers $(p, q)$. The lens space $L(p, q)$ admits a $U(1)$ action such that $L(p, q) / U(1)$ is uniformizable. The lens space $L(p, q)$ is given as the quotient space of $S^{3}$ by the $\boldsymbol{Z} / p \boldsymbol{Z}$ action defined by $\ell \cdot\left(z_{1}, z_{2}\right)=$ $\left(e^{2 \pi \ell / p \sqrt{-1}} z_{1}, e^{2 \pi \ell q / p \sqrt{-1}} z_{2}\right)$ for $\ell \in \boldsymbol{Z} / p \boldsymbol{Z}$. There is a $U(1)$ action (actually a $U(1) /(\boldsymbol{Z} / p \boldsymbol{Z})$ 
action) induced from the diagonal $U(1)$ action of $\left\{e^{2 \pi \sqrt{-1} t}\right\}$ on the unit sphere $S^{3}$. For this action $\underline{\Sigma}=L(p, q) / U(1)$ is the 2-dimensional sphere without cone points $(p=1)$ or with two cone points of angle $2 \pi / p(p \neq 1)$. Thus $\underline{\Sigma}$ is uniformizable and we can apply the previous argument.

If the fixed point set is not empty, we need the following lemma which is $\widetilde{S L}(2 ; \boldsymbol{R})$ version of Lemma 5.4.

Lemma 9.7. - Let $\widehat{A}: \boldsymbol{C} \longrightarrow \boldsymbol{C}$ be the map given in Lemma 5.4 preserving concentric circles, where we identify $\boldsymbol{R}^{2}$ with $\boldsymbol{C}$ and $a=a(w, \bar{w})$ is a real analytic function on $w \bar{w}$ and $a(0)=1$. If $a-1$ is divisible by $(w \bar{w})^{k}$, then the map $\widehat{A}_{k}: \boldsymbol{C} \longrightarrow \boldsymbol{C}$ satisfying $\left(\widehat{A}_{k}(w)\right)^{k}=$ $\widehat{A}\left(w^{k}\right)$ is real analytic at $(0,0)$.

Proof. - Put $z=w^{k}$ and in the coordinate $(z, \bar{z})$, the map $\widehat{A}$ given by

$$
\widehat{A}\left(\begin{array}{l}
x \\
y
\end{array}\right)=\frac{\sqrt{x^{2}+y^{2}}}{\sqrt{a^{2} x^{2}+a^{-2} y^{2}}}\left(\begin{array}{c}
a x \\
a^{-1} y
\end{array}\right)
$$

is written as follows:

$$
\begin{aligned}
& \widehat{A}(z)=\frac{\sqrt{z \bar{z}}}{\sqrt{a^{2} \frac{(z+\bar{z})^{2}}{4}-\frac{1}{a^{2}} \frac{(z-\bar{z})^{2}}{4}}}\left(a \frac{z+\bar{z}}{2}+\frac{1}{a} \frac{z-\bar{z}}{2}\right) \\
& =\frac{\sqrt{z \bar{z}}}{\sqrt{\frac{1}{2}\left(a^{2}+\frac{1}{a^{2}}\right) z \bar{z}+\frac{1}{4}\left(a^{2}-\frac{1}{a^{2}}\right)\left(z^{2}+\bar{z}^{2}\right)}}\left(\frac{1}{2}\left(a+\frac{1}{a}\right) z+\frac{1}{2}\left(a-\frac{1}{a}\right) \bar{z}\right) \\
& =\frac{\frac{1}{2}\left(a+\frac{1}{a}\right) z+\frac{1}{2}\left(a-\frac{1}{a}\right) \bar{z}}{\sqrt{\frac{1}{2}\left(a^{2}+\frac{1}{a^{2}}\right)+\frac{1}{4}\left(a^{2}-\frac{1}{a^{2}}\right) \frac{z^{2}+\bar{z}^{2}}{z \bar{z}}}} \\
& =\frac{z+\frac{1}{2} \frac{(a-1)^{2}}{a} z+\frac{1}{2} \frac{(a-1)(a+1)}{a} \bar{z}}{\sqrt{1+\frac{1}{2} \frac{(a-1)^{2}(a+1)^{2}}{a^{2}}+\frac{1}{4} \frac{(a-1)(a+1)\left(a^{2}+1\right)}{a^{2}} \frac{z^{2}+\bar{z}^{2}}{z \bar{z}}}} .
\end{aligned}
$$

If $a-1$ is divisible by $\left(x^{2}+y^{2}\right)^{k}=(w \bar{w})^{k}=z \bar{z}$, Then $\widehat{A}(z)$ is divisible by $z$ and written as the convergent series

$$
\widehat{A}(z)=z\left(1+\sum a_{i j} z^{i} \bar{z}^{j}\right) .
$$

The map $\widehat{A}_{k}$ is written as

$$
\widehat{A}_{k}(w)=\sqrt[k]{w^{k}\left(1+\sum a_{i j} w^{k i} \bar{w}^{k j}\right)}=w \sqrt[k]{1+\sum a_{i j} w^{k i} \bar{w}^{k j}}
$$

and it is real analytic.

If the fixed point set is not empty, $\underline{\Sigma}=M / U(1)$ is a surface of genus $g$ with boundary and with $\ell$ marked points. It is always uniformizable. Since $\underline{\Sigma}=M / U(1)$ is uniformizable, we proceed as before and we obtain a multi-section of $M \longrightarrow M / U(1)$ outside the boundary. 
For the boundary components corresponding to the fixed point set, we have a $k$-fold multisection. By using this multi-section we make $\widehat{A}_{k}$ act along the orbits. We can arrange the orbitwise rotation $f$ obtained by Proposition 9.1 to satisfy that $f$-id is divisible by $\left(x^{2}+y^{2}\right)^{2 k}$. We take a real analytic function $a$ on $\underline{\Sigma}$ such that $a=1$ on the boundary $\partial \underline{\Sigma}$, and $a>1$ on $\underline{\Sigma} \backslash \partial \underline{\Sigma}, a-1$ is divisible by $\left(x^{2}+y^{2}\right)^{k}$ along the boundary $\partial \underline{\Sigma}$ but the $(2 k)$-th derivative is not trivial. By using the real analytic map $\underline{\Sigma} \longrightarrow \widetilde{S L}^{k}(2 ; \boldsymbol{R})$ which lifts $w \longmapsto\left(\begin{array}{cc}a(w) & 0 \\ 0 & a(w)^{-1}\end{array}\right)$, by Lemma 3.1 the orbitwise rotation $f$ close to the identity can be written as a product of two commutators. Then by Lemmas 3.4 and 9.7, the diffeomorphisms appearing in the commutators are real analytic on the fixed point set.

Thus we proved Theorem 9.4.

\section{Appendix 1: Proof of Theorem 5.3}

Theorem 5.3 is a special case $(n=1)$ of the following Theorem 10.1. Before stating Theorem 10.1 , we clarify the situation.

We consider the complex vector space $\boldsymbol{C}^{n}$ with the coordinate $\left(z_{1}, \ldots, z_{n}\right)$. Let $U$ be a neighborhood of the origin 0 . Let $\Phi: U \longrightarrow \varphi(U) \subset C^{n}$ be a real analytic diffeomorphism fixing 0 such that $T_{0} \varphi=\operatorname{id}_{T_{0} \boldsymbol{C}^{n}}$.

Then the component $\varphi_{i}(z, \bar{z})(i=1, \ldots, n)$ of $\Phi(z, \bar{z})=\left(\varphi_{1}(z, \bar{z}), \ldots, \varphi_{n}(z, \bar{z})\right)$ is written as a convergent series in the variables $(z, \bar{z})=\left(\left(z_{1}, \ldots, z_{n}\right),\left(\bar{z}_{1}, \ldots, \bar{z}_{n}\right)\right)$, and satisfies $\frac{\partial \varphi_{i}}{\partial z_{j}}(0)=\delta_{i j}$ and $\frac{\partial \varphi_{i}}{\partial \bar{z}_{j}}(0)=0$. Hence the linear term of $\varphi_{j}$ is $z_{j}$.

Let $U(1) \times C^{n} \longrightarrow \boldsymbol{C}^{n}$ be the $U(1)$ action given by

$$
\left(e^{2 \pi \sqrt{-1} t},\left(z_{1}, \ldots, z_{n}\right)\right) \longmapsto e^{2 \pi \sqrt{-1} t} \cdot\left(z_{1}, \ldots, z_{n}\right)=\left(e^{2 \pi \sqrt{-1} m_{1} t} z_{1}, \ldots, e^{2 \pi \sqrt{-1} m_{n} t} z_{n}\right),
$$

where $\left(m_{1}, \ldots, m_{n}\right)$ is a primitive integer vector called the type of the $U(1)$ action. Any effective real analytic $U(1)$ action on $\boldsymbol{C}^{n}$ with the unique fixed point set $\{0\}$ is real analytically conjugate to this action for some $\left(m_{1}, \ldots, m_{n}\right)$.

Assume further that $\Phi(z, \bar{z})$ is on the orbit of $z$. Put

$$
\varphi_{j}(z, \bar{z})=\sum_{\substack{k \geq 0, \ell \geq 0 \\ k+\ell \geq 1}} g_{k \ell}^{j} z_{j}^{k} \bar{z}_{j}^{\ell},
$$

where $g_{k \ell}^{j}$ are convergent series on

$$
\left(\left(z_{1}, \ldots, z_{j-1}, z_{j+1}, \ldots, z_{n}\right),\left(\bar{z}_{1}, \ldots, \bar{z}_{j-1}, \bar{z}_{j+1}, \ldots, \bar{z}_{n}\right)\right) .
$$

Since $\varphi(z, \bar{z})$ is on the same orbit of $z, \varphi_{j}(z, \bar{z}) \overline{\varphi_{j}(z, \bar{z})}=z_{j} \bar{z}_{j}$. Since $\varphi_{j}(z, \bar{z}) \overline{\varphi_{j}(z, \bar{z})}$ begins with

$$
\begin{aligned}
& \left(g_{1,0}^{j} z_{j}+g_{0,1}^{j} \bar{z}_{j}+\cdots\right)\left(\bar{g}_{1,0}^{j} \bar{z}_{j}+\bar{g}_{0,1}^{j} z_{j}+\cdots\right) \\
& \quad=g_{1,0}^{j} \bar{g}_{0,1}^{j} z_{j}^{2}+\left(g_{1,0}^{j} \bar{g}_{1,0}^{j}+g_{0,1}^{j} \bar{g}_{0,1}^{j}\right) z_{j} \bar{z}_{j}+g_{0,1}^{j} \bar{g}_{1,0}^{j} \bar{z}_{j}{ }^{2}+\cdots
\end{aligned}
$$

and this coincides with $z_{j} \bar{z}_{j}, g_{1,0}^{j} \bar{g}_{0,1}^{j}=0, g_{1,0}^{j} \bar{g}_{1,0}^{j}+g_{0,1}^{j} \bar{g}_{0,1}^{j}=1$ and $g_{0,1}^{j} \bar{g}_{1,0}^{j}=0$. Here $g_{1,0}^{j}(0)=1$ and hence it is nonzero on a neighborhood of $0, g_{0,1}^{j}=0$ and $\left|g_{1,0}^{j}\right|=1$. If $g_{0,2}^{j}, \ldots, g_{0, \ell-1}^{j}$ is 0 and $g_{0, \ell}^{j} \neq 0$, then the coefficient of $\bar{z}_{j}^{\ell+1}$ of $\varphi_{j}(z, \bar{z}) \overline{\varphi_{j}(z, \bar{z})}$ is $g_{0, \ell}^{j}$. 
Since $\varphi_{j}(z, \bar{z}) \overline{\varphi_{j}(z, \bar{z})}=z_{j} \bar{z}_{j}, g_{0, \ell}^{j}=0$. Thus all the terms of $\varphi_{j}(z, \bar{z})$ contain $z_{j}$ and $\varphi_{j}(z, \bar{z})$ is written as $\varphi_{j}(z, \bar{z})=z_{j} u_{j}(z, \bar{z})$, where $u_{j}(z, \bar{z})$ is real analytic, $u_{j}(0,0)=1$ and $u_{j}(z, \bar{z}) \overline{u_{j}(z, \bar{z})}=1$. Then by putting $u_{j}(z, \bar{z})=1-v_{j}(z, \bar{z})$ and

$$
2 \pi \sqrt{-1} \mu_{i}(z, \bar{z})=\log \left(1-v_{j}(z, \bar{z})\right)=-\sum_{i=1}^{\infty} \frac{v_{j}(z, \bar{z})^{i}}{i}
$$

$\varphi_{j}(z, \bar{z})$ is written as $\varphi_{j}(z, \bar{z})=z_{j} e^{2 \pi \sqrt{-1}} \mu_{i}(z, \bar{z})$, where $\mu_{i}(z, \bar{z})$ is real-valued if the value of the variable $\bar{z}$ is the complex conjugate of $z$.

Now since $\Phi(z, \bar{z})=\left(z_{1} e^{2 \pi \sqrt{-1} \mu_{1}(z, \bar{z})}, \ldots, z_{n} e^{2 \pi \sqrt{-1} \mu_{n}(z, \bar{z})}\right)$ is on the same orbit as $z=\left(z_{1}, \ldots, z_{n}\right)$, and $\mu_{1}, \ldots, \mu_{n}$ are small near the origin,

$$
2 \pi \sqrt{-1} \mu_{j}(z, \bar{z}) / m_{j}=2 \pi \sqrt{-1} \mu_{k}(z, \bar{z}) / m_{k}
$$

holds where $\left|z_{j}\right| \cdot\left|z_{k}\right| \neq 0$. This means that the real analytic functions $\mu_{1}(z, \bar{z}) / m_{1}, \ldots$, $\mu_{n}(z, \bar{z}) / m_{n}$ defined on a neighborhood of 0 coincide on the open set where $\left|z_{1}\right| \cdots\left|z_{n}\right| \neq 0$. Hence there is a real analytic function $\mu(z, \bar{z})$ defined in a neighborhood of 0 such that $\mu_{1}(z, \bar{z})=m_{1} \mu(z, \bar{z}), \ldots, \mu_{n}(z, \bar{z})=m_{n} \mu(z, \bar{z})$. Thus the orbit preserving map $\Phi$ is written as follows:

$$
\Phi(z, \bar{z})=\left(z_{1} e^{2 \pi \sqrt{-1} m_{1} \mu(z, \bar{z})}, \ldots, z_{n} e^{2 \pi \sqrt{-1} m_{n} \mu(z, \bar{z})}\right) .
$$

We show the following theorem.

Theorem 10.1. - Let $\Delta_{\zeta}$ be the polydisk of radius $\zeta$. Let $\alpha \in \boldsymbol{R}$ be a Diophantine number. Let $\Phi(z, \bar{z} ; w)=\left(\varphi_{1}(z, \bar{z} ; w), \ldots, \varphi_{n}(z, \bar{z} ; w)\right)$ be a real analytic family of real analytic diffeomorphisms of a neighborhood $U$ of 0 in $\boldsymbol{C}^{n}$ which sends each orbit the $U(1)$ action of type $\left(m_{1}, \ldots, m_{n}\right)$ to itself, and $T_{(0)} \Phi_{w}=\operatorname{id}_{T_{0} \boldsymbol{C}^{n}}$, where $\Phi_{w}(z)=\Phi(z, \bar{z} ; w)$ and $w \in \Delta_{\zeta} \cap \boldsymbol{R}^{m}$. Then there are real analytic maps $\lambda: U \times\left(\Delta_{\zeta} \cap \boldsymbol{R}^{m}\right) \longrightarrow \boldsymbol{R}(\lambda(z, \bar{z} ; w)$ is constant along each orbit) and $H: U \times\left(\Delta_{\zeta} \cap \boldsymbol{R}^{m}\right) \longrightarrow U(H(z, \bar{z} ; w)$ is on the same orbit as $z)$ such that

$$
e^{-2 \pi \sqrt{-1} \lambda_{w}(z)} \cdot e^{2 \pi \sqrt{-1} \alpha} \cdot \Phi_{w}(z)=H_{w}\left(e^{2 \pi \sqrt{-1} \alpha} \cdot H_{w}{ }^{-1}(z)\right),
$$

where $\lambda_{w}(z)=\lambda_{w}(z, \bar{z} ; w)$ and $H_{w}(z)=H(z, \bar{z} ; w)$.

For the proof, we rewrite the equation. By replacing $H_{w}{ }^{-1}(z)$ by $z$, the equation is written as follows:

$$
e^{-2 \pi \sqrt{-1} \lambda_{w}\left(H_{w}(z)\right)} \cdot e^{2 \pi \sqrt{-1} \alpha} \cdot \Phi_{w}\left(H_{w}(z)\right)=H_{w}\left(e^{2 \pi \sqrt{-1} \alpha} \cdot z\right) .
$$

Since $\Phi_{w}(z)$ sends each orbit to itself, we have the real analytic function $\mu_{w}(z)=$ $\mu(z, \bar{z} ; w)$ such that

$$
\Phi_{w}(z)=\left(z_{1} e^{2 \pi \sqrt{-1} m_{1} \mu_{w}(z)}, \ldots, z_{n} e^{2 \pi \sqrt{-1} m_{n} \mu_{w}(z)}\right) .
$$

We are going to find $H_{w}(z)$ such that $T_{0} H_{w}=\operatorname{id}_{T_{0} C^{n}}$. If $H_{w}(z)$ is on the same orbit as $z$, then there is a real analytic function $k_{w}(z)=k(z, \bar{z} ; w)$ such that

$$
H_{w}(z)=\left(z_{1} e^{2 \pi \sqrt{-1} m_{1} k_{w}(z)}, \ldots, z_{n} e^{2 \pi \sqrt{-1} m_{n} k_{w}(z)}\right) .
$$


If $\lambda_{w}$ is constant along each orbit, $\lambda_{w}\left(H_{w}(z)\right)=\lambda_{w}(z)$. Then the $i$-th coordinate of the above equation is written as follows:

$$
\begin{gathered}
e^{-2 \pi \sqrt{-1} m_{i} \lambda_{w}(z)} e^{2 \pi \sqrt{-1} m_{i} \alpha}\left(z_{i} e^{2 \pi \sqrt{-1} m_{i} k_{w}(z)}\right) e^{2 \pi \sqrt{-1} m_{i} \mu_{w}\left(H_{w}(z)\right)} \\
=z_{i} e^{2 \pi \sqrt{-1} m_{i} \alpha} e^{2 \pi \sqrt{-1} m_{i} \mu_{w}\left(e^{2 \pi \sqrt{-1}} \cdot z\right)}
\end{gathered}
$$

Since $H_{w}(0)=0$ and $k_{w}(0)=\lambda_{w}(0)=\mu_{w}(0)=0$, we have the following equation.

$$
\begin{aligned}
\mu_{w}\left(H_{w}(z)\right)-\lambda_{w}(z) & =k_{w}\left(e^{2 \pi \sqrt{-1} \alpha} \cdot z\right)-k_{w}(z) \\
H_{w}(z) & =\left(z_{1} e^{2 \pi \sqrt{-1} m_{1} k_{w}(z)}, \ldots, z_{n} e^{2 \pi \sqrt{-1} m_{n} k_{w}(z)}\right) .
\end{aligned}
$$

We use the Kolmogorov-Arnold-Moser process to obtain the sequence converging to the solution.

Put

$$
G(\lambda, k)(z, \bar{z} ; w)=\mu_{w}\left(H_{w}(z)\right)-\lambda_{w}(z)-\left(k_{w}\left(e^{2 \pi \sqrt{-1} \alpha} \cdot z\right)-k_{w}(z)\right) .
$$

We are looking for $\lambda_{w}$ and $k_{w}$ such that $G\left(\lambda_{w}, k_{w}\right)(z, \bar{z} ; w)=0$. The question is:

"If we have an approximation $(\lambda, k)$ of the solution, how can we make a better approximation $(\lambda+\widehat{\lambda}, k+\widehat{k})$ ?"

In principle, for an appropriate norm $\|\cdot\|$, we have

$$
\left\|G(\lambda+\widehat{\lambda}, k+\widehat{k})-G(\lambda, k)-D G_{(\lambda, k)}(\widehat{\lambda}, \widehat{k})\right\| \leq \text { const }(\|\widehat{\lambda}\|+\|\widehat{k}\|)^{2} .
$$

Thus for the given $(\lambda, k)$, take $(\widehat{\lambda}, \widehat{k})$ so that $G(\lambda, k)+D G_{(\lambda, k)}(\widehat{\lambda}, \widehat{k})$ is small, then $(\lambda+\widehat{\lambda}, k+\widehat{k})$ should be a better approximation.

First we investigate the terms which we need to estimate.

Put

$$
\mu_{w}\left(z_{1}, \ldots, z_{n}, \theta\right)=\mu_{w}\left(z_{1} e^{2 \pi \sqrt{-1} m_{1} \theta}, \ldots, z_{n} e^{2 \pi \sqrt{-1} m_{n} \theta}\right)
$$

Then

$$
\frac{\partial \mu_{w}}{\partial \theta}=\sum_{i=1}^{n}\left(2 \pi \sqrt{-1} m_{i} z_{i} \frac{\partial \mu_{w}}{\partial z_{i}}-2 \pi \sqrt{-1} m_{i} \bar{z}_{i} \frac{\partial \mu_{w}}{\partial \bar{z}_{i}}\right) .
$$

The differential $D G_{(\lambda, k)}$ is computed as follows:

$$
D G_{(\lambda, k)}(\widehat{\lambda}, \widehat{k})=\frac{\partial \mu_{w}}{\partial \theta}\left(H_{w}(z)\right) \widehat{k}(z)-\widehat{\lambda}(z)-\left(\widehat{k}\left(e^{2 \pi \sqrt{-1} \alpha} \cdot z\right)-\widehat{k}(z)\right) .
$$

Then

$$
\begin{aligned}
G(\lambda+\widehat{\lambda}, k+ & \widehat{k})-G(\lambda, k)-D G_{(\lambda, k)}(\widehat{\lambda}, \widehat{k}) \\
= & \mu_{w}\left(z_{1} e^{2 \pi \sqrt{-1} m_{1}\left(k_{w}(z)+\widehat{k}_{w}(z)\right)}, \ldots, z_{n} e^{2 \pi \sqrt{-1} m_{n}\left(k_{w}(z)+\widehat{k}_{w}(z)\right)}\right) \\
& -\mu_{w}\left(z_{1} e^{2 \pi \sqrt{-1} m_{1} k_{w}(z)}, \ldots, z_{n} e^{2 \pi \sqrt{-1} m_{n} k_{w}(z)}\right) \\
& -\frac{\partial \mu}{\partial \theta}\left(z_{1} e^{2 \pi \sqrt{-1} m_{1} k_{w}(z)}, \ldots, z_{n} e^{2 \pi \sqrt{-1} m_{n} k_{w}(z)}\right) \widehat{k}(z) .
\end{aligned}
$$

Hence

$$
\left|G(\lambda+\widehat{\lambda}, k+\widehat{k})-G(\lambda, k)-D G_{(\lambda, k)}(\widehat{\lambda}, \widehat{k})\right| \leq \sup \left|\frac{\partial^{2} \mu}{\partial \theta^{2}}\right|(\sup |\widehat{k}|)^{2}
$$


Here $\frac{\partial^{2} \mu}{\partial \theta^{2}}=\left(\sum_{i=1}^{n}\left(2 \pi \sqrt{-1} m_{i} z_{i} \frac{\partial}{\partial z_{i}}-2 \pi \sqrt{-1} m_{i} \bar{z}_{i} \frac{\partial}{\partial \bar{z}_{i}}\right)\right)^{2} \mu$.

Put

$$
k_{w}\left(z_{1}, \ldots, z_{n}, \theta\right)=k_{w}\left(z_{1} e^{2 \pi \sqrt{-1} m_{1} \theta}, \ldots, z_{n} e^{2 \pi \sqrt{-1} m_{n} \theta}\right) .
$$

Then

$$
\frac{\partial k_{w}}{\partial \theta}=\sum_{i=1}^{n}\left(2 \pi \sqrt{-1} m_{i} z_{i} \frac{\partial k_{w}}{\partial z_{i}}-2 \pi \sqrt{-1} m_{i} \bar{z}_{i} \frac{\partial k_{w}}{\partial \bar{z}_{i}}\right) .
$$

Now put $\widehat{k}(z)=\left(1+\frac{\partial k_{w}}{\partial \theta}(z)\right) E(z)$, where $E(z)=E(z, \bar{z} ; w)$. By substituting this to the differential of $G(\lambda, k)$,

$$
\begin{aligned}
D G_{(\lambda, k)}(\widehat{\lambda},(1 & \left.\left.+\frac{\partial k_{w}}{\partial \theta}\right) E\right) \\
= & \frac{\partial \mu_{w}}{\partial \theta}\left(H_{w}(z)\right)\left(1+\frac{\partial k_{w}}{\partial \theta}(z)\right) E(z)-\widehat{\lambda}(z) \\
& -\left(\left(1+\frac{\partial k_{w}}{\partial \theta}\left(e^{2 \pi \sqrt{-1} \alpha} \cdot z\right)\right) E\left(e^{2 \pi \sqrt{-1} \alpha} \cdot z\right)-\left(1+\frac{\partial k_{w}}{\partial \theta}(z)\right) E(z)\right) .
\end{aligned}
$$

Since

$$
\begin{aligned}
G(\lambda, k)= & \mu_{w}\left(z_{1}, \ldots, z_{n}, \theta+k_{w}\left(z_{1}, \ldots, z_{n}, \theta\right)\right)-\lambda\left(z_{1}, \ldots, z_{n}\right) \\
& -\left(k_{w}\left(z_{1}, \ldots, z_{n}, \theta+\alpha\right)-k_{w}\left(z_{1}, \ldots, z_{n}, \theta\right)\right) \\
\frac{\partial G(\lambda, k)}{\partial \theta}(z)= & \frac{\partial \mu_{w}}{\partial \theta}\left(H_{w}(z)\right)\left(1+\frac{\partial k_{w}}{\partial \theta}(z)\right)-\left(\frac{\partial k_{w}}{\partial \theta}\left(e^{2 \pi \sqrt{-1} \alpha} \cdot z\right)-\frac{\partial k_{w}}{\partial \theta}(z)\right) .
\end{aligned}
$$

Hence we have

$$
\begin{aligned}
\frac{\partial G(\lambda, k)}{\partial \theta}(z) E(z)= & \frac{\partial \mu_{w}}{\partial \theta}\left(H_{w}(z)\right)\left(1+\frac{\partial k_{w}}{\partial \theta}(z)\right) E(z) \\
& -\left(\frac{\partial k_{w}}{\partial \theta}\left(e^{2 \pi \sqrt{-1} \alpha} \cdot z\right) E(z)-\frac{\partial k_{w}}{\partial \theta}(z) E(z)\right) \\
= & \frac{\partial \mu_{w}}{\partial \theta}\left(H_{w}(z)\right)\left(1+\frac{\partial k_{w}}{\partial \theta}(z)\right) E(z) \\
& -\left(\left(1+\frac{\partial k_{w}}{\partial \theta}\left(e^{2 \pi \sqrt{-1} \alpha} \cdot z\right)\right) E(z)-\left(1+\frac{\partial k_{w}}{\partial \theta}(z)\right) E(z)\right)
\end{aligned}
$$

Thus

$$
\begin{aligned}
D G_{(\lambda, k)}\left(\widehat{\lambda},\left(1+\frac{\partial k_{w}}{\partial \theta}\right) E\right)-\frac{\partial G(\lambda, k)}{\partial \theta}(z) E(z) & \\
& =-\left(1+\frac{\partial k_{w}}{\partial \theta}\left(e^{2 \pi \sqrt{-1} \alpha} \cdot z\right)\right)\left(E\left(e^{2 \pi \sqrt{-1} \alpha} \cdot z\right)-E(z)\right)-\widehat{\lambda}(z) .
\end{aligned}
$$

For $(\lambda, k)$, we would like to solve

$$
G(\lambda, k)-\widehat{\lambda}=\left(1+\frac{\partial k_{w}}{\partial \theta}\left(e^{2 \pi \sqrt{-1} \alpha} \cdot z\right)\right)\left(E\left(e^{2 \pi \sqrt{-1} \alpha} \cdot z\right)-E(z)\right) .
$$

That is

$$
E\left(e^{2 \pi \sqrt{-1} \alpha} \cdot z\right)-E(z)=\left(1+\frac{\partial k_{w}}{\partial \theta}\left(e^{2 \pi \sqrt{-1} \alpha} \cdot z\right)\right)^{-1}(G(\lambda, k)-\widehat{\lambda})
$$


To find $E$, it is necessary that

$$
\int\left(1+\frac{\partial k_{w}}{\partial \theta}\left(e^{2 \pi \sqrt{-1} \alpha} \cdot z\right)\right)^{-1}(G(\lambda, k)-\widehat{\lambda}) \mathrm{d} \theta=0
$$

Since $\widehat{\lambda}$ is constant on each orbit, this is rewritten as

$$
\int\left(1+\frac{\partial k_{w}}{\partial \theta}\left(e^{2 \pi \sqrt{-1} \alpha} \cdot z\right)\right)^{-1} G(\lambda, k) \mathrm{d} \theta=\hat{\lambda} \int\left(1+\frac{\partial k_{w}}{\partial \theta}\left(e^{2 \pi \sqrt{-1} \alpha} \cdot z\right)\right)^{-1} \mathrm{~d} \theta
$$

With these $\left(\widehat{\lambda}, \widehat{k}=\left(1+\frac{\partial k_{w}}{\partial \theta}\right) E\right)$,

$$
\begin{aligned}
& G(\lambda, k)+D G_{(\lambda, k)}\left(\hat{\lambda},\left(1+\frac{\partial k_{w}}{\partial \theta}\right) E\right) \\
&= G(\lambda, k)+D G_{(\lambda, k)}\left(\widehat{\lambda},\left(1+\frac{\partial k_{w}}{\partial \theta}\right) E\right)-\frac{\partial G(\lambda, k)}{\partial \theta}(z) E(z) \\
&+\frac{\partial G(\lambda, k)}{\partial \theta}(z) E(z) \\
&= G(\lambda, k)-\left(1+\frac{\partial k_{w}}{\partial \theta}\left(e^{2 \pi \sqrt{-1} \alpha} \cdot z\right)\right)\left(E\left(e^{2 \pi \sqrt{-1} \alpha} \cdot z\right)-E(z)\right)-\hat{\lambda} \\
&+\frac{\partial G(\lambda, k)}{\partial \theta}(z) E(z) \\
&= \frac{\partial G(\lambda, k)}{\partial \theta}(z) E(z) .
\end{aligned}
$$

It is important to note that since $G(\lambda, k)$ is small, $\frac{\partial G(\lambda, k)}{\partial \theta}(z)$ is small.

Thus by (10.1) and (10.2),

$$
|G(\lambda+\widehat{\lambda}, k+\widehat{k})| \leq \sup \left|\frac{\partial G(\lambda, k)}{\partial \theta}(z)\right| \sup |E(z)|+\sup \left|\frac{\partial^{2} \mu_{w}}{\partial \theta^{2}}\right|(\sup |\widehat{k}|)^{2} .
$$

We treat the equation

$$
E\left(e^{2 \pi \sqrt{-1} \alpha} \cdot z\right)-E(z)=\left(1+\frac{\partial k}{\partial \theta}\left(e^{2 \pi \sqrt{-1} \alpha} \cdot z\right)\right)^{-1}(G(\lambda, k)-\widehat{\lambda})
$$

for the given $(\lambda, k)$, where $\widehat{\lambda}$ is already determined.

Assume that

$$
\sup _{\Delta_{\eta} \times \Delta_{\eta} \times \Delta_{\zeta}}|G(\lambda, k)| \leq \varepsilon, \quad \text { and } \quad \sup _{\Delta_{\eta} \times \Delta_{\eta} \times \Delta_{\zeta}}\left|\frac{\partial k}{\partial \theta}(z, \bar{z} ; w)\right| \leq \frac{1}{2^{2}} .
$$

Then by the equation determining $\widehat{\lambda}, \sup _{\Delta_{\eta} \times \Delta_{\eta} \times \Delta_{\zeta}}|\widehat{\lambda}| \leq \frac{2^{4}}{3^{2}} \varepsilon$, hence

$$
\sup _{\Delta_{\eta} \times \Delta_{\eta} \times \Delta_{\zeta}}\left|\left(1+\frac{\partial k_{w}}{\partial \theta}\left(e^{2 \pi \sqrt{-1} \alpha} \cdot z\right)\right)^{-1}(G(\lambda, k)-\widehat{\lambda})\right| \leq \frac{4}{3} \frac{5^{2}}{3^{2}} \varepsilon<2^{2} \varepsilon .
$$


Here

$$
\begin{aligned}
\nu(z, \bar{z} ; w) & =\left(1+\frac{\partial k_{w}}{\partial \theta}\left(e^{2 \pi \sqrt{-1} \alpha} \cdot z\right)\right)^{-1}(G(\lambda, k)-\widehat{\lambda}) \\
& =\sum_{j_{1}, \ldots, j_{n}, k_{1}, \ldots, k_{n}} a_{j_{1} \cdots j_{n} k_{1} \cdots k_{n}}(w) z_{1}{ }^{j_{1}} \cdots z_{n}{ }^{j_{n}} \bar{z}_{1} k_{1} \ldots \bar{z}_{n}{ }^{k_{n}}
\end{aligned}
$$

satisfies $a_{j_{1} \cdots j_{n} k_{1} \cdots k_{n}}(w)=\overline{a_{k_{1} \cdots k_{n} j_{1} \cdots j_{n}}(w)}$ and $a_{j_{1} \cdots j_{n} k_{1} \cdots k_{n}}(w)=0$ if $\sum_{i=1}^{n} m_{i} j_{i}=$ $\sum_{i=1}^{n} m_{i} k_{i}$. The reason for the latter is as follows: Since the integral of $\nu$ along the orbit is 0 ,

$$
\begin{aligned}
\int \nu\left(e^{2 \pi \sqrt{-1} \theta} \cdot z, e^{-2 \pi \sqrt{-1} \theta} \cdot \bar{z} ; w\right) \mathrm{d} \theta & \\
= & \sum_{j_{1}, \ldots, j_{n}, k_{1}, \ldots, k_{n}} \int e^{2 \pi \sqrt{-1}\left(\sum_{i=1}^{n} m_{i} j_{i}-\sum_{i=1}^{n} m_{i} k_{i}\right) \theta} z_{1}^{j_{1}} \cdots z_{n}^{j_{n}} \bar{z}_{1}{ }^{k_{1}} \cdots \bar{z}_{n}{ }^{k_{n}} \mathrm{~d} \theta \\
& =\sum_{\sum_{i=1}^{n} m_{i} j_{i}=\sum_{i=1}^{n} m_{i} k_{i}} a_{j_{1} \cdots j_{n} k_{1} \cdots k_{n}}(w) z_{1}{ }^{j_{1}} \cdots z_{n}{ }^{j_{n}} \bar{z}_{1}{ }^{k_{1}} \ldots \bar{z}_{n}{ }^{k_{n}}=0 .
\end{aligned}
$$

Since it vanishes as a function on $(z, \bar{z} ; w)$, the coefficient satisfies $a_{j_{1} \cdots j_{n} k_{1} \cdots k_{n}}(w)=0$ if $\sum_{i=1}^{n} m_{i} j_{i}=\sum_{i=1}^{n} m_{i} k_{i}$

Since $\sup _{\Delta_{\eta} \times \Delta_{\eta} \times \Delta_{\zeta}}|\nu(z, \bar{z} ; w)| \leq 2^{2} \varepsilon,\left|a_{j_{1} \cdots j_{n} k_{1} \cdots k_{n}}(w)\right| \leq \frac{2^{2} \varepsilon}{\eta \sum_{i=1}^{n} j_{i}+\sum_{i=1}^{n} k_{i}}$ by the Cauchy inequality.

For the series $\nu(z, \bar{z} ; w)=\sum_{j_{1}, \ldots, j_{n}, k_{1}, \ldots, k_{n}} a_{j_{1} \cdots j_{n} k_{1} \cdots k_{n}}(w) z_{1}{ }^{j_{1}} \cdots z_{n}{ }^{j_{n}} \bar{z}_{1}{ }^{k_{1}} \cdots \bar{z}_{n}{ }^{k_{n}}$ with $a_{j_{1} \cdots j_{n} k_{1} \cdots k_{n}}(w)=\overline{a_{k_{1} \cdots k_{n} j_{1} \cdots j_{n}}(w)}$ and $a_{j_{1} \cdots j_{n} k_{1} \cdots k_{n}}(w)=0$ for $\sum_{i=1}^{n} m_{i} j_{i}=\sum_{i=1}^{n} m_{i} k_{i}$, put

$$
E(z)=\sum_{j_{1}, \ldots, j_{n}, k_{1}, \ldots, k_{n}} b_{j_{1} \cdots j_{n} k_{1} \cdots k_{n}}(w) z_{1}^{j_{1}} \cdots z_{n}^{j_{n}} \bar{z}_{1}^{k_{1}} \cdots \bar{z}_{n}{ }^{k_{n}}
$$

and we can solve

$$
E\left(e^{2 \pi \sqrt{-1} \alpha} \cdot z\right)-E(z)=\nu(z, \bar{z} ; w)
$$

Since

$$
\begin{gathered}
E\left(e^{2 \pi \sqrt{-1} \alpha} \cdot z\right)-E(z)=\sum_{j_{1}, \ldots, j_{n}, k_{1}, \ldots, k_{n}}\left(e^{2 \pi \sqrt{-1} \alpha\left(\sum_{i=1}^{n} m_{i} j_{i}-\sum_{i=1}^{n} m_{i} k_{i}\right)}-1\right) \\
\cdot b_{j_{1} \cdots j_{n} k_{1} \cdots k_{n}}(w) z_{1}^{j_{1}} \cdots z_{n}^{j_{n}} \bar{z}_{1} k_{1} \cdots \bar{z}_{n}^{k_{n}} \\
b_{j_{1} \cdots j_{n} k_{1} \cdots k_{n}}(w)=\frac{a_{j_{1} \cdots j_{n} k_{1} \cdots k_{n}}(w)}{e^{2 \pi \sqrt{-1} \alpha\left(\sum_{i=1}^{n} m_{i} j_{i}-\sum_{i=1}^{n} m_{i} k_{i}\right)}-1} .
\end{gathered}
$$


Thus

$$
\begin{aligned}
E(z) & =\sum_{j_{1}, \ldots, j_{n}, k_{1}, \ldots, k_{n}} b_{j_{1} \cdots j_{n} k_{1} \cdots k_{n}}(w) z_{1}^{j_{1}} \cdots z_{n}^{j_{n}} \bar{z}_{1}^{k_{1}} \cdots \bar{z}_{n}^{k_{n}} \\
& =\sum_{j_{1}, \ldots, j_{n}, k_{1}, \ldots, k_{n}} \frac{a_{j_{1} \cdots j_{n} k_{1} \cdots k_{n}}(w)}{e^{2 \pi \sqrt{-1} \alpha\left(\sum_{i=1}^{n} m_{i} j_{i}-\sum_{i=1}^{n} m_{i} k_{i}\right)}-1} z_{1}^{j_{1}} \cdots z_{n}^{j_{n}} \bar{z}_{1} k_{1} \cdots \bar{z}_{n}{ }^{k_{n}} .
\end{aligned}
$$

This $E(z)$ is real if the value of the variable $\bar{z}$ is the complex conjugate of the value of $z$ and satisfies

$$
\nu(z, \bar{z} ; w)=E\left(e^{2 \pi \sqrt{-1} \alpha} \cdot z\right)-E(z) .
$$

Note that by the Diophantine condition,

$$
\left|e^{2 \pi \sqrt{-1} \alpha\left(\sum_{i=1}^{n} m_{i} j_{i}-\sum_{i=1}^{n} m_{i} k_{i}\right)}-1\right| \geq \frac{C_{\beta}}{\left|\sum_{i=1}^{n} m_{i} j_{i}-\sum_{i=1}^{n} m_{i} k_{i}\right|^{1+\beta}} \quad(\beta \geq 1) .
$$

Since $\left|a_{j_{1} \cdots j_{n} k_{1} \cdots k_{n}}(w)\right| \leq \frac{2^{2} \varepsilon}{\eta \sum_{i=1}^{n} j_{i}+\sum_{i=1}^{n} k_{i}}$,

$$
\left|b_{j_{1} \cdots j_{n} k_{1} \cdots k_{n}}(w)\right| \leq \frac{2^{2} \varepsilon}{C_{\beta}} \frac{\left|\sum_{i=1}^{n} m_{i} j_{i}-\sum_{i=1}^{n} m_{i} k_{i}\right|^{1+\beta}}{\eta^{\sum_{i=1}^{n} j_{i}+\sum_{i=1}^{n} k_{i}}}
$$

holds. Then, for $|z| \leq(1-a) \eta(0<a<1)$,

$$
\begin{aligned}
\sum_{j_{i} \geq 0, k_{i} \geq 0}\left|b_{j_{1} \cdots j_{n} k_{1} \cdots k_{n}}(w)\right|\left|z_{1}^{j_{1}} \cdots z_{n}{ }^{j_{n}} \bar{z}_{1}^{k_{1}} \cdots \bar{z}_{n}{ }^{k_{n}}\right| & \\
& \leq \sum_{j_{i} \geq 0, k_{i} \geq 0} \frac{2^{2} \varepsilon}{C_{\beta}}\left|\sum_{i=1}^{n} m_{i} j_{i}-\sum_{i=1}^{n} m_{i} k_{i}\right|^{1+\beta}(1-a)^{\sum_{i=1}^{n} j_{i}+\sum_{i=1}^{n} k_{i}} \\
& \leq \sum_{j_{i} \geq 0, k_{i} \geq 0} \frac{2^{2} \varepsilon}{C_{\beta}}\left(\sum_{i=1}^{n}\left|m_{i}\right|\right)^{1+\beta}\left(\sum_{i=1}^{n}\left|j_{i}-k_{i}\right|\right)^{1+\beta}(1-a)^{\sum_{i=1}^{n} j_{i}+\sum_{i=1}^{n} k_{i}} .
\end{aligned}
$$

Here

$$
\begin{aligned}
\sum_{j_{i} \geq 0, k_{i} \geq 0} & \left(\sum_{i=1}^{n}\left|j_{i}-k_{i}\right|\right)^{1+\beta}(1-a)^{\sum_{i=1}^{n} j_{i}+\sum_{i=1}^{n} k_{i}} \\
& \leq \sum_{j_{i} \geq 0, k_{i} \geq 0, \sum_{i=1}^{n} j_{i}+\sum_{i=1}^{n} k_{i}>0}\left(\sum_{i=1}^{n}\left(j_{i}+k_{i}\right)\right)^{1+\beta}(1-a)^{\sum_{i=1}^{n} j_{i}+\sum_{i=1}^{n} k_{i}} \\
& \leq \sum_{\ell \geq 1}\left(\begin{array}{c}
2 n-1+\ell \\
2 n-1
\end{array}\right) \ell^{1+\beta}(1-a)^{\ell} \\
& \leq 2^{2 n-1} \sum_{\ell \geq 1} \ell^{2 n+\beta}(1-a)^{\ell}
\end{aligned}
$$


In the second inequality above we used the following inequality:

$$
\frac{(2 n-1+\ell)(2 n-2+\ell) \cdots(1+\ell)}{(2 n-1)(2 n-2) \cdots 1 \cdot \ell^{2 n-1}}=\left(\frac{1}{\ell}+\frac{1}{2 n-1}\right)\left(\frac{1}{\ell}+\frac{1}{2 n-2}\right) \cdots\left(\frac{1}{\ell}+\frac{1}{1}\right) \leq 2^{2 n-1} .
$$

Then by using the equalities

$$
\int_{0}^{\infty} x^{2 n+\beta} e^{-\gamma x} \mathrm{~d} x=\int_{0}^{\infty}\left(\frac{x}{\gamma}\right)^{2 n+\beta} e^{-x} \mathrm{~d}\left(\frac{x}{\gamma}\right)=\frac{\Gamma(2 n+1+\beta)}{\gamma^{2 n+1+\beta}}
$$

with $\gamma=|\log (1-a)|$ and the inequality $|\log (1-a)| \geq a$,

$$
\sum_{j_{i} \geq 0, k_{i} \geq 0}\left(\sum_{i=1}^{n}\left|j_{i}-k_{i}\right|\right)^{1+\beta}(1-a)^{\sum_{i=1}^{n} j_{i}+\sum_{i=1}^{n} k_{i}} \leq 2^{2 n-1} \frac{\Gamma(2 n+1+\beta)}{a^{2 n+1+\beta}}
$$

Thus

$$
\begin{aligned}
\sum_{j_{i} \geq 0, k_{i} \geq 0}\left|b_{j_{1} \cdots j_{n} k_{1} \cdots k_{n}}(w)\right|\left|z_{1}^{j_{1}} \cdots z_{n}{ }^{j_{n}} \bar{z}_{1}{ }^{k_{1}} \cdots \bar{z}_{n}{ }^{k_{n}}\right| \\
\leq \frac{2^{2} \varepsilon}{C_{\beta}}\left(\sum_{i=1}^{n}\left|m_{i}\right|\right)^{1+\beta} 2^{2 n-1} \frac{\Gamma(2 n+1+\beta)}{a^{2 n+1+\beta}} \\
=\frac{2^{2 n+1} \Gamma(2 n+1+\beta)}{C_{\beta}}\left(\sum_{i=1}^{n}\left|m_{i}\right|\right)^{1+\beta} \frac{\varepsilon}{a^{2 n+1+\beta}} .
\end{aligned}
$$

This implies that $E$ is real analytic on $\Delta_{(1-a) \eta} \times \Delta_{(1-a) \eta} \times \Delta_{\zeta}$ and

$$
\sup _{\Delta_{(1-a) \eta} \times \Delta_{(1-a) \eta} \times \Delta_{\zeta}}|E| \leq \frac{2^{2 n+1} \Gamma(2 n+1+\beta)}{C_{\beta}}\left(\sum_{i=1}^{n}\left|m_{i}\right|\right)^{1+\beta} \frac{\varepsilon}{a^{2 n+1+\beta}} .
$$

Since

$$
\begin{aligned}
& \frac{\partial E}{\partial \theta}=2 \pi \sqrt{-1} \sum_{j_{1}, \ldots, j_{n}, k_{1}, \ldots, k_{n}}\left(\sum_{i=1}^{n} m_{i} j_{i}-\sum_{i=1}^{n} m_{i} k_{i}\right) \\
& \cdot b_{j_{1} \cdots j_{n} k_{1} \cdots k_{n}}(w) z_{1}^{j_{1}} \cdots z_{n}{ }^{j_{n}} \bar{z}_{1}{ }^{k_{1}} \cdots \bar{z}_{n}{ }^{k_{n}}
\end{aligned}
$$




$$
\begin{aligned}
& \text { for }\left|z_{i}\right| \leq(1-a) \eta(i=1, \ldots, n) \text {, } \\
& \left|\frac{\partial E}{\partial \theta}\right| \leq 2 \pi \sum_{j_{i} \geq 0, k_{i} \geq 0} \frac{2^{2} \varepsilon}{C_{\beta}}\left|\sum_{i=1}^{n} m_{i} j_{i}-\sum_{i=1}^{n} m_{i} k_{i}\right|^{2+\beta}(1-a)^{\sum_{i=1}^{n} j_{i}+\sum_{i=1}^{n} k_{i}} \\
& \leq \frac{2^{3} \pi \varepsilon}{C_{\beta}} \sum_{j_{i} \geq 0, k_{i} \geq 0}\left(\sum_{i=1}^{n}\left|m_{i}\right|\right)^{2+\beta}\left(\sum_{i=1}^{n}\left|j_{i}-k_{i}\right|\right)^{2+\beta}(1-a)^{\sum_{i=1}^{n} j_{i}+\sum_{i=1}^{n} k_{i}} \\
& \leq \frac{2^{3} \pi \varepsilon}{C_{\beta}}\left(\sum_{i=1}^{n}\left|m_{i}\right|\right)^{2+\beta} \sum_{\sum_{j} j_{i} \geq 0, k_{i} \geq 0,}\left(\sum_{i=1}^{n}\left(j_{i}+k_{i}\right)\right)^{2+\beta}(1-a)^{\sum_{i=1}^{n} j_{i}+\sum_{i=1}^{n} k_{i}} \\
& \leq \frac{2^{3} \pi \varepsilon}{C_{\beta}}\left(\sum_{i=1}^{n}\left|m_{i}\right|\right)^{2+\beta} 2^{2 n-1} \sum_{\ell>0} \ell^{2 n+1+\beta}(1-a)^{\ell} \\
& \leq \frac{2^{3} \pi \varepsilon}{C_{\beta}}\left(\sum_{i=1}^{n}\left|m_{i}\right|\right)^{2+\beta} 2^{2 n-1} \frac{\Gamma(2 n+2+\beta)}{a^{2 n+2+\beta}} \text {. }
\end{aligned}
$$

Thus

$$
\sup _{\Delta_{(1-a) \eta} \times \Delta_{(1-a) \eta} \times \Delta_{\zeta}}\left|\frac{\partial E}{\partial \theta}\right| \leq \frac{2^{2 n+2} \pi \Gamma(2 n+2+\beta)}{C_{\beta}}\left(\sum_{i=1}^{n}\left|m_{i}\right|\right)^{2+\beta} \frac{\varepsilon}{a^{2 n+2+\beta}} .
$$

Thus by putting $C_{\beta}^{\prime}=\frac{2^{2 n+1} \Gamma(2 n+2+\beta)}{C_{\beta}}$ and $M=\sum_{i=1}^{n}\left|m_{i}\right|$, we have

$$
\sup _{\Delta_{(1-a) \eta} \times \Delta_{(1-a) \eta} \times \Delta_{\zeta}}|E| \leq \frac{C_{\beta}^{\prime} M^{1+\beta} \varepsilon}{a^{2 n+1+\beta}}
$$

and

$$
\sup _{\Delta_{(1-a) \eta} \times \Delta_{(1-a) \eta} \times \Delta_{\zeta}}\left|\frac{\partial E}{\partial \theta}\right| \leq \frac{2 \pi C_{\beta}^{\prime} M^{2+\beta} \varepsilon}{a^{2 n+2+\beta}} .
$$

Then for $\widehat{k}=\left(1+\frac{\partial k_{w}}{\partial \theta}\right) E$,

$$
\sup _{\Delta_{(1-a) \eta} \times \Delta_{(1-a) \eta} \times \Delta_{\zeta}}|\widehat{k}| \leq \frac{5 C_{\beta}^{\prime} M^{1+\beta} \varepsilon}{4 a^{2 n+1+\beta}} .
$$

If a holomorphic function $f$ on $\Delta_{\eta} \times \Delta_{\eta} \times \Delta_{\zeta}$ satisfies $\sup _{\Delta_{\eta} \times \Delta_{\eta} \times \Delta_{\zeta}}|f(z, \bar{z}, w)| \leq K$, then for

$$
\frac{\partial f}{\partial \theta}(z, \bar{z}, w)=\sum_{i=1}^{n}\left(2 \pi \sqrt{-1} m_{i} z_{i} \frac{\partial f}{\partial z_{i}}-2 \pi \sqrt{-1} m_{i} \bar{z}_{i} \frac{\partial f}{\partial \bar{z}_{i}}\right)
$$


$\sup _{\Delta_{(1-a) \eta} \times \Delta_{(1-a) \eta} \times \Delta_{\zeta}}\left|\frac{\partial f}{\partial z_{i}}(z, \bar{z}, w)\right|$ and $\sup _{\Delta_{(1-a) \eta} \times \Delta_{(1-a) \eta} \times \Delta_{\zeta}}\left|\frac{\partial f}{\partial \bar{z}_{i}}(z, \bar{z}, w)\right|$ are estimated by $\frac{K}{a \eta}$ by the Cauchy formula, and

$$
\sup _{\Delta_{(1-a) \eta} \times \Delta_{(1-a) \eta} \times \Delta_{\zeta}}\left|\frac{\partial f}{\partial \theta}(z, \bar{z}, w)\right| \leq \frac{2^{2}(1-a) \pi K}{a} \sum_{i=1}^{n}\left|m_{i}\right| \leq \frac{2^{2} \pi K}{a} \sum_{i=1}^{n}\left|m_{i}\right| .
$$

For

$$
\begin{aligned}
\frac{\partial^{2} f}{\partial \theta^{2}}(z, \bar{z}, w)= & \left(\sum_{i=1}^{n}\left(2 \pi \sqrt{-1} m_{i} z_{i} \frac{\partial}{\partial z_{i}}-2 \pi \sqrt{-1} m_{i} \bar{z}_{i} \frac{\partial}{\partial \bar{z}_{i}}\right)\right)^{2} f \\
= & -2^{2} \pi^{2}\left(\sum_{i=1}^{n} m_{i}{ }^{2}\left(z_{i} \frac{\partial}{\partial z_{i}}+z_{i}{ }^{2} \frac{\partial^{2}}{\partial z_{i}{ }^{2}}\right)+m_{i}{ }^{2}\left(\bar{z}_{i} \frac{\partial}{\partial \bar{z}_{i}}+\bar{z}_{i}{ }^{2} \frac{\partial^{2}}{\partial \bar{z}_{i}{ }^{2}}\right)\right. \\
& +\sum_{i \neq j}\left(m_{i} m_{j} z_{i} z_{j} \frac{\partial^{2}}{\partial z_{i} \partial z_{j}}+m_{i} m_{j} \bar{z}_{i} \bar{z}_{j} \frac{\partial^{2}}{\partial \bar{z}_{i} \partial \bar{z}_{j}}\right) \\
& \left.-\sum_{i, j}\left(m_{i} m_{j} z_{i} \bar{z}_{j} \frac{\partial^{2}}{\partial z_{i} \partial \overline{z_{j}}}+m_{i} m_{j} \bar{z}_{i} z_{j} \frac{\partial^{2}}{\partial \bar{z}_{i} \partial z_{j}}\right)\right) f
\end{aligned}
$$

by the Cauchy formula,

$$
\begin{aligned}
\sup _{\Delta_{\eta} \times \Delta_{\eta} \times \Delta_{\zeta}} \mid & \frac{\partial^{2} f}{\partial \theta^{2}}(z, \bar{z}, w) \mid \\
\leq & 2^{2} \pi^{2}\left(2 \sum_{i=1}^{n}\left|m_{i}\right|^{2}\left(\frac{1-a}{a}+\frac{2(1-a)^{2}}{a^{2}}\right) K\right. \\
& \left.+2 \sum_{i \neq j}\left|m_{i} m_{j}\right| \frac{(1-a)^{2}}{a^{2}} K+2 \sum_{i, j}\left|m_{i} m_{j}\right| \frac{(1-a)^{2}}{a^{2}} K\right) \\
\leq & 2^{2} \pi^{2}\left(2 \sum_{i=1}^{n}\left|m_{i}\right|^{2}\left(\frac{1-a}{a}+\frac{(1-a)^{2}}{a^{2}}\right) K+4 \sum_{i, j}\left|m_{i} m_{j}\right| \frac{(1-a)^{2}}{a^{2}} K\right) \\
\leq & 2^{2} \pi^{2} \cdot 6\left(\sum_{i=1}^{n}\left|m_{i}\right|\right)^{2} \frac{K}{a^{2}}
\end{aligned}
$$

Thus if $\sup _{\Delta_{\eta} \times \Delta_{\eta} \times \Delta_{\zeta}}\left|\frac{\partial k}{\partial \theta}(z, \bar{z}, w)\right| \leq \frac{1}{2^{2}}$, then for

$$
\begin{gathered}
\frac{\partial^{2} k}{\partial \theta^{2}}(z, \bar{z}, w)=\sum_{i=1}^{n}\left(2 \pi \sqrt{-1} m_{i} z_{i} \frac{\partial}{\partial z_{i}}-2 \pi \sqrt{-1} m_{i} \bar{z}_{i} \frac{\partial}{\partial \bar{z}_{i}}\right) \frac{\partial k}{\partial \theta}, \\
\sup _{\Delta_{(1-a) \eta} \times \Delta_{(1-a) \eta} \times \Delta_{\zeta}}\left|\frac{\partial^{2} k}{\partial \theta^{2}}(z, \bar{z}, w)\right| \leq \frac{\pi}{a} \sum_{i=1}^{n}\left|m_{i}\right|=\frac{\pi M}{a} .
\end{gathered}
$$

Since $\frac{\partial \widehat{k}}{\partial \theta}=\frac{\partial^{2} k}{\partial \theta^{2}} E+\left(1+\frac{\partial k}{\partial \theta}\right) \frac{\partial E}{\partial \theta}$,

$$
\sup _{\Delta_{(1-a) \eta} \times \Delta_{(1-a) \eta} \times \Delta_{\zeta}}\left|\frac{\partial \widehat{k}}{\partial \theta}\right| \leq \frac{\pi M}{a} \frac{C_{\beta}^{\prime} M^{1+\beta} \varepsilon}{a^{2 n+1+\beta}}+\frac{5}{4} \frac{2 \pi C_{\beta}^{\prime} M^{2+\beta} \varepsilon}{a^{2 n+2+\beta}} \leq \frac{2^{2} \pi C_{\beta}^{\prime} M^{2+\beta} \varepsilon}{a^{2 n+2+\beta}} .
$$


Assume that $\sup _{\Delta_{\eta} \times \Delta_{\eta} \times \Delta_{\zeta}}|\mu| \leq \varepsilon^{\prime}$. Then for

$$
\begin{gathered}
\frac{\partial^{2} \mu}{\partial \theta^{2}}=\left(\sum_{i=1}^{n}\left(2 \pi \sqrt{-1} m_{i} z_{i} \frac{\partial}{\partial z_{i}}-2 \pi \sqrt{-1} m_{i} \bar{z}_{i} \frac{\partial}{\partial \bar{z}_{i}}\right)\right)^{2} \mu \\
\sup _{\Delta_{(1-a) \eta} \times \Delta_{(1-a) \eta} \times \Delta_{\zeta}}\left|\frac{\partial^{2} \mu}{\partial \theta^{2}}\right| \leq 2^{2} \pi^{2} \cdot 6\left(\sum_{i=1}^{n}\left|m_{i}\right|\right)^{2} \frac{\varepsilon^{\prime}}{a^{2}}=\frac{2^{3} 3 \pi^{2} M^{2} \varepsilon^{\prime}}{a^{2}} .
\end{gathered}
$$

Thus

$$
\begin{aligned}
\left(\sup _{\Delta_{(1-a) \eta} \times \Delta_{(1-a) \eta} \times \Delta_{\zeta}}\left|\frac{\partial^{2} \mu}{\partial \theta^{2}}\right|\right)\left(\sup _{\Delta_{(1-a) \eta} \times \Delta_{(1-a) \eta} \times \Delta_{\zeta}}|\widehat{k}|\right)^{2} \\
\leq \frac{2^{3} 3 \pi^{2} M^{2} \varepsilon^{\prime}}{a^{2}}\left(\frac{5 C_{\beta}^{\prime} M^{1+\beta} \varepsilon}{4 a^{2 n+1+\beta}}\right)^{2}=\frac{3 \cdot 5^{2} \pi^{2} C_{\beta}^{\prime 2} M^{4+2 \beta} \varepsilon^{\prime} \varepsilon^{2}}{2 a^{4 n+4+2 \beta}} .
\end{aligned}
$$

Since $\sup _{\Delta_{\eta} \times \Delta_{\eta} \times \Delta_{\zeta}}|G(\lambda, k)| \leq \varepsilon$

$$
\sup _{\Delta_{(1-a) \eta} \times \Delta_{(1-a) \eta} \times \Delta_{\zeta}}\left|\frac{\partial G(\lambda, k)}{\partial \theta}\right| \leq \frac{2^{2} \pi}{a}\left(\sum_{i=1}^{n}\left|m_{i}\right|\right) \varepsilon=\frac{2^{2} \pi M \varepsilon}{a}
$$

and

$$
\left(\sup _{\Delta_{(1-a) \eta} \times \Delta_{(1-a) \eta} \times \Delta_{\zeta}}\left|\frac{\partial G(\lambda, k)}{\partial \theta}\right|\right)\left(\sup _{\Delta_{(1-a) \eta} \times \Delta_{(1-a) \eta} \times \Delta_{\zeta}}|E|\right) \leq \frac{2^{2} \pi C_{\beta}^{\prime} M^{2+\beta} \varepsilon^{2}}{a^{2 n+2+\beta}} .
$$

We assume further that

$$
3 \cdot 5^{2} \pi C_{\beta}^{\prime} M^{2+\beta} \varepsilon^{\prime} \leq 2^{3}
$$

and we have

$$
\sup _{\Delta_{(1-a) \eta} \times \Delta_{(1-a) \eta} \times \Delta_{\zeta}}|G(\lambda+\widehat{\lambda}, k+\widehat{k})| \leq \frac{2^{3} \pi C_{\beta}^{\prime} M^{2+\beta}}{a^{4 n+4+2 \beta}} \varepsilon^{2} .
$$

Now we put $\lambda_{0}=0$ and $k_{0}=0$. Then $G\left(\lambda_{0}, k_{0}\right)=G(0,0)=\mu(z)$ and assume that

$$
\sup _{\Delta_{\eta} \times \Delta_{\eta} \times \Delta_{\zeta}}|\mu(z)| \leq \varepsilon=\varepsilon^{\prime} \leq \frac{2^{3}}{3 \cdot 5^{2} \pi C_{\beta}^{\prime} M^{2+\beta}} .
$$

Put $\eta_{\ell-1}=\left(\frac{1}{2}+\frac{1}{2^{\ell}}\right) \eta$ and $\varepsilon_{0}=\varepsilon$, we have

$$
\sup _{\Delta_{\eta_{0}} \times \Delta_{\eta_{0}} \times \Delta_{\zeta}}\left|G\left(\lambda_{0}, k_{0}\right)\right| \leq \varepsilon_{0} .
$$

Note that

$$
\eta_{\ell}=\eta_{\ell-1} \frac{\frac{1}{2}+\frac{1}{2^{\ell+1}}}{\frac{1}{2}+\frac{1}{2^{\ell}}}=\eta_{\ell-1}\left(1-a_{\ell-1}\right)
$$

where $a_{\ell-1}=\frac{\frac{1}{2^{\ell+1}}}{\frac{1}{2}+\frac{1}{2^{\ell}}} \geq \frac{1}{2^{\ell+1}}$ for $\ell \geq 1$.

$4^{\mathrm{e}}$ SÉRIE - TOME $42-2009-\mathrm{N}^{\circ} 4$ 
Then we have $\left(\widehat{\lambda}_{0}, \widehat{k}_{0}=\left(1+\frac{\partial k_{0}}{\partial \theta}\right) E_{0}=E_{0}\right)$ such that

$$
G\left(\lambda_{0}, k_{0}\right)-\widehat{\lambda}_{0}=\left(1+\frac{\partial k_{0}}{\partial \theta}\left(e^{2 \pi \sqrt{-1} \alpha} z\right)\right)\left(E_{0}\left(e^{2 \pi \sqrt{-1} \alpha} z\right)-E_{0}(z)\right)
$$

and

$$
\widehat{k}_{0}(z)=\left(1+\frac{\partial k_{0}}{\partial \theta}(z)\right) E_{0}(z)
$$

where

$$
\begin{aligned}
\sup _{\Delta_{\eta_{0}} \times \Delta_{\eta_{0}} \times \Delta_{\zeta}}\left|\widehat{\lambda}_{0}\right| & \leq \frac{2^{4}}{3^{2}} \varepsilon_{0} \\
\sup _{\Delta_{\eta_{1}} \times \Delta_{\eta_{1}} \times \Delta_{\zeta}}\left|\widehat{k}_{0}\right| & \leq \frac{5 C_{\beta}^{\prime} M^{1+\beta}}{4 a_{0}^{2 n+1+\beta} \varepsilon_{0}} \\
\sup _{\Delta_{\eta_{1}} \times \Delta_{\eta_{1}} \times \Delta_{\zeta}}\left|\frac{\partial \widehat{k}_{0}}{\partial \theta}\right| & \leq \frac{2^{2} \pi C_{\beta}^{\prime} M^{2+\beta}}{a_{0}^{2 n+2+\beta}} \varepsilon_{0} .
\end{aligned}
$$

Then for $\lambda_{1}=\lambda_{0}+\widehat{\lambda}_{0}$ and $k_{1}=k_{0}+\widehat{k}_{0}$,

$$
\begin{aligned}
\sup _{\Delta_{\eta_{1}} \times \Delta_{\eta_{1}} \times \Delta_{\zeta}}\left|G\left(\lambda_{1}, k_{1}\right)\right| & \leq \frac{2^{3} \pi C_{\beta}^{\prime} M^{2+\beta}}{a_{0}{ }^{4 n+4+2 \beta}} \varepsilon_{0}{ }^{2} \\
& \leq 2^{3} \pi C_{\beta}^{\prime} M^{2+\beta} 2^{2(4 n+4+2 \beta)} \varepsilon_{0}{ }^{2} .
\end{aligned}
$$

For $\left(\lambda_{\ell}, k_{\ell}\right)$, assume that

$$
\sup _{\Delta_{\eta_{\ell}} \times \Delta_{\eta_{\ell}} \times \Delta_{\zeta}}\left|G\left(\lambda_{\ell}, k_{\ell}\right)\right| \leq \varepsilon_{\ell} .
$$

Then we get $\left(\widehat{\lambda}_{\ell}, \widehat{k}_{\ell}=\left(1+\frac{\partial k}{\partial \theta}\right) E_{\ell}\right)$ such that

$$
G\left(\lambda_{\ell}, k_{\ell}\right)-\widehat{\lambda}_{\ell}=\left(1+\frac{\partial k_{\ell}}{\partial \theta}\left(e^{2 \pi \sqrt{-1} \alpha} z\right)\right)\left(E_{\ell}\left(e^{2 \pi \sqrt{-1} \alpha} z\right)-E_{\ell}(z)\right)
$$

and

$$
\widehat{k}_{\ell}(z)=\left(1+\frac{\partial k_{\ell}}{\partial \theta}(z)\right) E_{\ell}(z)
$$

where

$$
\begin{aligned}
\sup _{\Delta_{\eta_{\ell}} \times \Delta_{\eta_{\ell}} \times \Delta_{\zeta}}\left|\widehat{\lambda}_{\ell}\right| & \leq \frac{2^{4}}{3^{2}} \varepsilon_{\ell} \\
\sup _{\Delta_{\eta_{n+1}} \times \Delta_{\eta_{n+1}} \times \Delta_{\zeta}}\left|\widehat{k}_{\ell}\right| & \leq \frac{5 C_{\beta}^{\prime} M^{1+\beta}}{4 a_{\ell}^{2 n+1+\beta}} \varepsilon_{\ell} \\
\sup _{\Delta_{\eta_{\ell+1}} \times \Delta_{\eta_{\ell+1}} \times \Delta_{\zeta}}\left|\frac{\partial \widehat{k}_{\ell}}{\partial \theta}\right| & \leq \frac{2^{2} \pi C_{\beta}^{\prime} M^{2+\beta}}{a_{\ell}^{2 n+2+\beta}} \varepsilon_{\ell} .
\end{aligned}
$$


Put $\lambda_{\ell+1}=\lambda_{\ell}+\widehat{\lambda}_{\ell}$ and $k_{\ell+1}=k_{\ell}+\widehat{k}_{\ell}$. Then

$$
\begin{aligned}
\sup _{\Delta_{\ell+1} \times \Delta_{\eta_{\ell+1}} \times \Delta_{\zeta}}\left|G\left(\lambda_{\ell+1}, k_{\ell+1}\right)\right| & \leq \frac{2^{3} \pi C_{\beta}^{\prime} M^{2+\beta}}{a_{\ell}^{4 n+4+2 \beta}} \varepsilon_{\ell}^{2} \\
& \leq 2^{3} \pi C_{\beta}^{\prime} M^{2+\beta} 2^{(n+2)(4 n+4+2 \beta)} \varepsilon_{\ell}^{2} .
\end{aligned}
$$

Moreover,

$$
\sup _{\Delta_{\eta_{n+1}} \times \Delta_{\eta_{n+1}} \times \Delta_{\zeta}}\left|\frac{\partial k_{n+1}}{\partial \theta}\right| \leq 2^{2} \pi C_{\beta}^{\prime} M^{2+\beta}\left(\frac{\varepsilon_{0}}{a_{0}^{2 n+2+\beta}}+\cdots+\frac{\varepsilon_{\ell}}{a_{\ell}^{2 n+2+\beta}}\right) .
$$

We will show that $\lambda_{\ell}$ and $k_{\ell}$ are defined successively and $\left(\lambda_{\ell}, k_{\ell}\right)$ converges to $(\lambda, k)$ which are holomorphic on $\Delta_{\eta / 2} \times \Delta_{\eta / 2} \times \Delta_{\zeta}$, and then we will have $G_{\mu}(\lambda, k)=0$.

Put $N=2^{3} \pi C_{\beta}^{\prime} M^{2+\beta} 2^{2(4 n+4+2 \beta)}$, and we have

$$
\varepsilon_{1} \leq N \varepsilon_{0}^{2}, \varepsilon_{2} \leq N 2^{4 n+4+2 \beta} \varepsilon_{1}^{2}, \ldots, \varepsilon_{\ell+1} \leq N 2^{n(4 n+4+2 \beta)} \varepsilon_{\ell}^{2}, \ldots
$$

If we take $\varepsilon_{0} \leq \frac{1}{N 2^{4 n+4+2 \beta}}$, then $\varepsilon_{1} \leq \frac{1}{N 2^{2(4 n+4+2 \beta)}}$. If $\varepsilon_{\ell} \leq \frac{1}{N 2^{(\ell+1)(4 n+4+2 \beta)}}$, then $\varepsilon_{\ell+1} \leq \frac{1}{N 2^{(\ell+2)(4 n+4+2 \beta)}}$. Then

$$
\begin{aligned}
2^{2} \pi C_{\beta}^{\prime} M^{2+\beta} & \left(\frac{\varepsilon_{0}}{a_{0}^{2 n+2+\beta}}+\cdots+\frac{\varepsilon_{\ell}}{a_{\ell}^{2 n+2+\beta}}\right) \\
& \leq 2^{2} \pi C_{\beta}^{\prime} M^{2+\beta}\left(\frac{2^{2(2 n+2+\beta)}}{N 2^{(4 n+4+2 \beta)}}+\cdots+\frac{2^{(\ell+2)(2 n+2+\beta)}}{N 2^{(\ell+1)(4 n+4+2 \beta)}}\right) \\
& =\frac{2^{2} \pi C_{\beta}^{\prime} M^{2+\beta}}{N}\left(1+\cdots+\frac{1}{2^{\ell(2 n+2+\beta)}}\right) \\
& \leq \frac{2^{3} \pi C_{\beta}^{\prime} M^{2+\beta}}{N}=\frac{2^{3} \pi C_{\beta}^{\prime} M^{2+\beta}}{2^{3} \pi C_{\beta}^{\prime} M^{2+\beta} 2^{2(4 n+4+2 \beta)}}=\frac{1}{2^{2(4 n+4+2 \beta)}} \leq \frac{1}{2^{2}},
\end{aligned}
$$

and

$$
\sup _{\Delta_{\eta_{n+1}} \times \Delta_{\eta_{n+1}} \times \Delta_{\zeta}}\left|\frac{\partial k_{n+1}}{\partial \theta}\right| \leq \frac{1}{2^{2}}
$$

is satisfied.

Thus the construction of the sequence $\left(\lambda_{\ell}, k_{\ell}\right)$ is performed. Since $\sum \varepsilon_{\ell}$ converges, $\lambda=\sum_{\ell=0}^{\infty} \widehat{\lambda}_{\ell}$ is holomorphic on $\Delta_{\eta / 2} \times \Delta_{\eta / 2} \times \Delta_{\zeta}$. Since

$$
\sum \frac{\varepsilon_{\ell}}{a_{\ell}^{2 n+1+\beta}}=\sum \frac{2^{(\ell+2)(2 n+1+\beta)}}{N 2^{(\ell+1)(4 n+4+2 \beta)}}=\sum \frac{1}{N 2^{2+\ell(2 n+3+\beta)}}
$$

converges, $k=\sum_{\ell=0}^{\infty} \widehat{k}_{\ell}$ is holomorphic on $\Delta_{\eta / 2} \times \Delta_{\eta / 2} \times \Delta_{\zeta}$.

Thus $(\lambda, k)$ satisfies $G(\lambda, k)=0$ on $\Delta_{\eta / 2} \times \Delta_{\eta / 2} \times \Delta_{\zeta}$ and this is the desired real analytic solution.

$4^{\text {e }}$ SÉRIE - TOME $42-2009-\mathrm{N}^{\mathrm{o}} 4$ 


\section{Appendix 2: Preliminary on the real analytic diffeomorphisms}

In this section, we review several fundamental facts on real analytic manifolds, real analytic maps and diffeomorphisms. Basic references are Cartan [4], Grauert-Remmert [7].

Let $\mathcal{A}$ be the sheaf of germs of real analytic functions on a real analytic manifold $M$. Analytically coherent sheaves are defined as follows. A sheaf $\mathcal{S}$ on $M$ is analytically coherent if there is a neighborhood $U$ of $x$, such that $\mathcal{S}_{U}$ is finitely generated over $\mathcal{A}_{U}$ and for $y \in U$, $\mathcal{S}_{y}$ is represented as follows:

$$
\mathcal{S}_{y}=\left(\mathcal{A}_{y} e_{1}+\cdots+\mathcal{A}_{y} e_{k}\right) /\left\{f=\sum_{i=1}^{k} a_{i y} e_{i} \mid \sum_{i=1}^{k} b_{i y}^{(j)} a_{i y}=0(j=1, \ldots, \ell)\right\}
$$

for some basis $\left\{e_{1}, \ldots, e_{k}\right\}$ and $b_{i j}^{(k)} \in \mathcal{A}_{U}$. In other words, there is an exact sequence of sheaves:

$$
\left(\mathcal{A}_{U}\right)^{\ell} \longrightarrow\left(\mathcal{A}_{U}\right)^{k} \longrightarrow \mathcal{S}_{U} \longrightarrow 0 .
$$

It is fundamental that if $\mathcal{S}_{x}$ is finitely generated over $\mathcal{A}_{x}$, then there is a neighborhood $U$ of $x$ where $\mathcal{S}_{U}$ is finitely generated over $\mathcal{A}_{U}$.

Let $H^{n}(M ; \mathcal{S})$ be the $n$-dimensional cohomology group with coefficients in $\mathcal{S}$. Then, Theorems A and B of Cartan are stated as follows ([4]).

Theorem 11.1 (Theorem A). - For any analytically coherent sheaf $\mathcal{S}, H^{0}(M ; \mathcal{S}) \longrightarrow \mathcal{S}_{x}$ is surjective.

Theorem 11.2 (Theorem B). - For any analytically coherent sheaf $\mathcal{S}$, for $n>0$, $H^{n}(M ; \mathcal{S})=0$.

Let $M$ be a real analytic submanifold in $\boldsymbol{R}^{N}$. Let $\mathcal{I}_{M}$ denote the sheaf of the real analytic functions on $\boldsymbol{R}^{N}$ which vanishes on $M$. Then, we have the well known proposition.

Proposition 11.3. - $\mathcal{I}_{M}$ is coherent.

For a real analytic submanifold $M$ of $\boldsymbol{R}^{N}$, the sheaf $\mathcal{A}_{M}$ of real analytic functions on $M$ is identified with the quotient $\mathcal{A} / \mathcal{I}_{M}$. Proposition 11.3 and Theorem $\mathrm{B}$ of Cartan (Theorem 11.2) imply the following proposition.

Proposition 11.4. - For any real analytic function $f$ on $M \subset \boldsymbol{R}^{N}$, there is an extension $\widetilde{f}$ on $\boldsymbol{R}^{N}$.

Proposition 11.5. - Any compact real analytic manifold $M$ is defined by $f=0$ with $f \in H^{0}\left(\boldsymbol{R}^{N} ; \mathcal{A}\right)$.

Proof. - Let $S^{N} \subset \boldsymbol{R}^{N+1}$ be the unit sphere in $\boldsymbol{R}^{N+1}$. Since $S^{N}-\{*\} \cong \boldsymbol{R}^{N}$ real analytically, $M$ is real analytically embedded in $S^{N} \subset \boldsymbol{R}^{N+1}$. It is sufficient to show that there is a real analytic function $f$ on $S^{N}$ defining $M$.

By Theorem A of Cartan (Theorem 11.1), for $x \in M$, the defining ideal $\left(\mathcal{I}_{M}\right)_{x}$ is generated by $F_{1}^{(x)}, \ldots, F_{k_{x}}^{(x)} \in H^{0}\left(\boldsymbol{R}^{N+1} ; \mathcal{I}_{M}\right)$. Then $G^{(x)}=\sum_{i=1}^{k_{x}}\left(F_{i}^{(x)}\right)^{2}$ is a real analytic function on $\boldsymbol{R}^{N+1}$ such that $G^{(x)}=0$ on $M$ and $G^{(x)}$ defines $M$ on a neighborhood $U_{x}$ of $x$. For 
$y \in S^{N} \backslash M,\left(\mathcal{I}_{M}\right)_{y}$ is generated by any $F^{(y)} \in H^{0}\left(\boldsymbol{R}^{N+1} ; \mathcal{I}_{M}\right)$ such that $F^{(y)} \neq 0$ on a neighborhood $U_{y}$ of $y$.

Now take the covering $\left\{U_{x}\right\}_{x \in M} \cup\left\{U_{y}\right\}_{y \in S^{N} \backslash M}$ of $S^{N}$. Since $S^{N}$ is compact, we have a finite subcovering $\left\{U_{x_{j}}\right\}_{j=1, \ldots, p} \cup\left\{U_{y_{\ell}}\right\}_{\ell=1, \ldots, q}$ of it. Then the sum $\sum_{j=1}^{p} G^{\left(x_{j}\right)}+\sum_{\ell=1}^{q}\left(F^{\left(y_{\ell}\right)}\right)^{2}$ is the desired defining function of $M$ on $S^{N}$.

The defining function $f_{M}$ obtained by Proposition 11.5 is positive and bounded. By taking a function like $\left(1-\frac{f_{M}}{K}\right)^{m}$, we obtain the following corollary.

Corollary 11.6. - For any $\varepsilon>0$ and $\delta>0$, there is a real analytic function $f$ on $\boldsymbol{R}^{N}$ which is 1 on $M$ and $f<\varepsilon$ out of the $\delta$ neighborhood of $M$.

Now we look at the topology of the set of real analytic maps (see Royden [18], Hirsch [12]).

It is shown by Grauert [6] and Morrey [14] that an $n$-dimensional real analytic manifold $M^{n}$ is real analytically embedded in the $(2 n+1)$-dimensional Euclidean space $\boldsymbol{R}^{2 n+1}$.

Let $M_{1}$ and $M_{2}$ be compact real analytic manifolds. Let $\operatorname{Map}^{r}\left(M_{1}, M_{2}\right)$ and $\operatorname{Map}^{\omega}\left(M_{1}, M_{2}\right)$ be the set of $C^{r}$ maps $(1 \leq r<\infty)$ and real analytic maps from $M_{1}$ to $M_{2}$, respectively.

Proposition 11.7. - For compact real analytic manifolds $M_{1}$ and $M_{2}, \operatorname{Map}^{\omega}\left(M_{1}, M_{2}\right)$ is dense in $\operatorname{Map}^{r}\left(M_{1}, M_{2}\right)$ in the $C^{r}$ topology.

Proof. - Using the real analytic embedding theorem, this is shown as follows. Let $M_{1}$ and $M_{2}$ be real analytic submanifolds of $\boldsymbol{R}^{N_{1}}$ and $\boldsymbol{R}^{N_{2}}$, respectively. Let $f: M_{1} \longrightarrow M_{2}$ be a $C^{r}$ map. Then the $C^{r}$ map $f$ extends to a $C^{r}$ map $\widetilde{f}: U_{M_{1}} \longrightarrow M_{2}$ for a neighborhood $U_{M_{1}}$ of $M_{1}$ in $\boldsymbol{R}^{N_{1}}$. Then by the Weierstrass approximation theorem, this $\tilde{f}$ is $C^{r}$ approximated by a $C^{\omega}$ map $\widehat{f}: U_{M_{1}} \longrightarrow \boldsymbol{R}^{N_{2}}$. Here, the trouble is that $\widehat{f}\left(M_{1}\right)$ may not be in $M_{2}$. Let $p_{M_{2}}$ denote the normal bundle projection for $M_{2} \subset \boldsymbol{R}^{N_{2}}$ which is defined in a neighborhood of $M_{2}$ and $C^{\omega}$. Then $p_{M_{2}} \circ \widehat{f}$ is a $C^{\omega}$ map to $M_{2}$ which is $C^{r}$ close to $f$.

For the group of real analytic diffeomorphisms of a compact real analytic manifold $M$, we have the following corollary.

COROllary 11.8. - For a compact real analytic manifold $M$, Diff ${ }^{\omega}(M)$ is dense in $\operatorname{Diff}^{r}(M)(1 \leq r \leq \infty)$ in the $C^{r}$ topology.

Proof. - Since $\operatorname{Diff}^{\omega}(M)$ is open in $\operatorname{Map}^{\omega}(M, M)$ in the $C^{r}$ topology, if $f$ is a diffeomorphism then $p_{M_{2}} \circ \widehat{f}$ in the proof of Proposition 11.7 is a $C^{\omega}$ diffeomorphism. Thus $\operatorname{Diff}^{\omega}(M)$ is dense in $\operatorname{Diff}^{r}(M)$ in the $C^{r}$ topology. For $r=\infty$, the $C^{\infty}$ topology is given as the projective limit topology. Hence $\operatorname{Diff}^{\omega}(M)$ is dense in $\operatorname{Diff}^{\infty}(M)$ in the $C^{\infty}$ topology.

Proposition 11.9. - For a compact real analytic manifold $M$, Diff ${ }^{\omega}(M)$ is locally contractible.

$4^{\mathrm{e}}$ SÉRIE - TOME $42-2009-\mathrm{N}^{\mathrm{o}} 4$ 
Proof. - Let $M \subset \boldsymbol{R}^{N}$, and let $p: \nu M \longrightarrow M$ be the projection of the normal bundle of $M$ to $M$. For a real analytic diffeomorphism $f$ in a $C^{1}$ neighborhood of the identity id ${ }_{M}$, take $f_{t}(x)=p((1-t) x+t f(x))$. Then $f_{t}(x)$ is a $C^{\omega}$ path in $\operatorname{Diff}^{\omega}(M)$ from the identity $\operatorname{id}_{M}$ to $f$.

Proposition 11.10. - For a compact real analytic manifold $M$, Diff ${ }^{\omega}(M)$ is homotopy equivalent to $\operatorname{Diff}^{r}(M)(1 \leq r \leq \infty)$.

Proof. - Given a continuous map $S^{k} \longrightarrow \operatorname{Diff}^{1}(M)$, it is approximated by a $C^{1}$ map $S^{k} \times M \longrightarrow M$ such that $\{*\} \times M \longrightarrow M$ are diffeomorphisms. Then it is approximated by a $C^{\omega}$ map $S^{k} \times M \longrightarrow M$ such that $\{*\} \times M \longrightarrow M$ are diffeomorphisms. Since $\operatorname{Diff}^{1}(M)$ is open in $\operatorname{Map}^{1}(M, M), \pi_{k}$ is surjective.

Given a continuous map $f: S^{k} \longrightarrow \operatorname{Diff}^{\omega}(M)$, assume that $f: S^{k} \longrightarrow \operatorname{Diff}^{\omega}(M)$ extends to a continuous map $F: D^{k+1} \longrightarrow \operatorname{Diff}^{1}(M) . F$ is approximated by $C^{1}$ map $D^{k+1} \times M \longrightarrow M$ such that $\{*\} \times M \longrightarrow M$ are diffeomorphisms. Then this is approximated by a $C^{\omega}$ map $\widehat{F}: D^{k+1} \times M \longrightarrow M$. Thus we have $\widehat{F}: D^{k+1} \longrightarrow \operatorname{Diff}^{\omega}(M)$. Now consider the map $S^{k} \times[0,1] \longrightarrow \operatorname{Diff}^{\omega}(M)$ given by $p_{M} \circ(t \widehat{F}+(1-t) f)$ and we attach these 2 maps along $S^{k}=\partial D^{k+1}$ to obtain a map $D^{k+1} \longrightarrow \operatorname{Diff}^{\omega}(M)$ bounded by $f$.

Proposition 11.11. - Let $L \subset M$ be a closed submanifold. If $f \in \operatorname{Diff}^{\omega}(L)$ extends to $F \in \operatorname{Diff}^{1}(M)$ then $f \in \operatorname{Diff}^{\omega}(L)$ extends to $\widehat{F} \in \operatorname{Diff}^{\omega}(M)$.

Proof. - We may assume that $M$ is a real analytic submanifold of $\boldsymbol{R}^{N}$. First we approximate $F$ by a real analytic map $F_{1}: M \longrightarrow M$. The $C^{1}$ norm of $F_{1} \mid L-f: L \longrightarrow \boldsymbol{R}^{N}$ can be made arbitrarily small. Let $p_{M}$ be the normal bundle projection on a neighborhood in $\boldsymbol{R}^{N}$ of $M$ to $M$. In the product $M \times[0,1] \subset \boldsymbol{R}^{N} \times[0,1]$, we consider the submanifold

$$
\left\{\left(p_{M}\left((1-t) F_{1}(x)-t f(x)\right), t\right) \mid x \in L, t \in[0,1]\right\} \subset M \times[0,1],
$$

which is $C^{\omega}$ diffeomorphic to $L \times[0,1]$. We have a vector field

$$
\left(\frac{\partial p_{M}\left((1-t) F_{1}(x)-t f(x)\right)}{\partial t}, 1\right)
$$

along this submanifold. Then by Proposition 11.4, we can extend this vector field to a $C^{\omega}$ vector field on $\boldsymbol{R}^{N} \times[0,1]$. The second component of the vector field is always taken to be 1 . Now we take the restriction of this vector field on $M \times[0,1]$ and take the image by $\left(\left(p_{M}\right)_{*}, \mathrm{id}_{*}\right)$ of the restriction. Then we have a $C^{\omega}$ vector field $\xi$ on $M \times[0,1]$, and for $x \in L$, $\left\{\left(p_{M}\left((1-t) F_{1}(x)-t f(x)\right), t\right) \mid t \in[0,1]\right\}$ is the integral curve of $\xi$. Let $\varphi: M \longrightarrow M$ be the time one map of $\xi$, then this is a diffeomorphism extending $f \circ\left(F_{1} \mid L\right)^{-1}$. Then $\varphi \circ F_{1}$ is a $C^{\omega}$ extension of $f$.

Corollary 11.12. - Let $L_{1} \subset M_{1}$ be a closed submanifold. If $f \in \operatorname{Diff}^{\omega}\left(L_{1} \times M_{2}\right)$ extends to $F \in \operatorname{Diff}^{1}\left(M_{1} \times M_{2}\right)$ then $f \in \operatorname{Diff}^{\omega}\left(L_{1} \times M_{2}\right)$ extends to $\widehat{F} \in \operatorname{Diff}^{\omega}\left(M_{1} \times M_{2}\right)$.

This corollary means the following. Given a $C^{\omega}$ map $f: S^{k} \longrightarrow \operatorname{Diff}^{\omega}(M)$, assume that $f: S^{k} \longrightarrow \operatorname{Diff}^{\omega}(M)$ extends to a continuous map $F: D^{k+1} \longrightarrow \operatorname{Diff}^{1}(M)$. Then $f$ extends to a $C^{\omega} \operatorname{map} \widehat{F}: D^{k+1} \longrightarrow \operatorname{Diff}^{\omega}(M)$. 


\section{Acknowlegement}

The author is grateful to the referee for his very careful reading and for his suggestions on the improvement of this paper.

\section{REFERENCES}

[1] V. I. ArnOL'D, Small denominators. I. Mapping the circle onto itself, Izv. Akad. Nauk SSSR Ser. Mat. 25 (1961), 21-86, (= Amer. Math. Soc. Translations 46 (1965), $213-$ 284).

[2] A. Banyaga, The structure of classical diffeomorphism groups, Mathematics and its Applications 400, Kluwer Academic Publishers Group, 1997.

[3] H. Cartan, Idéaux de fonctions analytiques de $n$ variables complexes, Ann. Sci. École Norm. Sup. 61 (1944), 149-197.

[4] H. Cartan, Variétés analytiques réelles et variétés analytiques complexes, Bull. Soc. Math. France 85 (1957), 77-99.

[5] D. B. A. Epstein, The simplicity of certain groups of homeomorphisms, Compositio Math. 22 (1970), 165-173.

[6] H. Grauert, On Levi's problem and the imbedding of real-analytic manifolds, Ann. of Math. 68 (1958), 460-472.

[7] H. Grauert, R. Remmert, Coherent analytic sheaves, Grund. Math. Wiss. 265, Springer, 1984.

[8] R. C. Gunning, Introduction to holomorphic functions of several variables. Vol. II, The Wadsworth \& Brooks/Cole Mathematics Series, 1990.

[9] S. Haller, J. Teichmann, Smooth perfectness through decomposition of diffeomorphisms into fiber preserving ones, Ann. Global Anal. Geom. 23 (2003), 53-63.

[10] M.-R. Herman, Simplicité du groupe des difféomorphismes de classe $C^{\infty}$, isotopes à l'identité, du tore de dimension n, C. R. Acad. Sci. Paris 273 (1971), 232-234.

[11] M.-R. Herman, Sur le groupe des difféomorphismes R-analytiques du tore, in Differential topology and geometry (Proc. Colloq., Dijon, 1974), Springer Lecture Notes in Math. 484, 1975, 36-42.

[12] U. Hirsch, Some remarks on analytic foliations and analytic branched coverings, Math. Ann. 248 (1980), 139-152.

[13] J. Mather, On the homology of Haefliger's classifying space, C.I.M.E. Differential Topology (1976), 71-116.

[14] C. B. Morrey JR., The analytic embedding of abstract real-analytic manifolds, Ann. of Math. 68 (1958), 159-201.

[15] J. Moser, A rapidly convergent iteration method and non-linear partial differential equations. I and II, Ann. Scuola Norm. Sup. Pisa 20 (1966), 265-315 and 499-535.

[16] P. Orlik, F. Raymond, Actions of $\mathrm{SO}(2)$ on 3-manifolds, in Proc. Conf. on Transformation Groups (New Orleans, La, 1967), Springer, 1968, 297-318.

[17] F. Raymond, Classification of the actions of the circle on 3-manifolds, Trans. Amer. Math. Soc. 131 (1968), 51-78.

$4^{\mathrm{e}}$ SÉRIE - TOME $42-2009-\mathrm{N}^{\mathrm{o}} 4$ 
[18] H. L. Royden, The analytic approximation of differentiable mappings, Math. Ann. 139 (1960), 171-179.

[19] C. L. Siegel, Iteration of analytic functions, Ann. of Math. 43 (1942), 607-612.

[20] W. Thurston, Foliations and groups of diffeomorphisms, Bull. Amer. Math. Soc. 80 (1974), 304-307.

(Manuscrit reçu le 2 juillet 2008;

accepté, après révision, le 24 février 2009.)

Takashi TsuboI

Graduate School of Mathematical Sciences

University of Tokyo

Komaba Meguro

Tokyo 153-8914, Japan

E-mail: tsuboi@ms.u-tokyo.ac.jp 